\title{
Developing
}

Microfluidic Tooling for 3D Cell-Culture

$\omega$

$\frac{\Omega}{\frac{D}{\bar{D}}}$
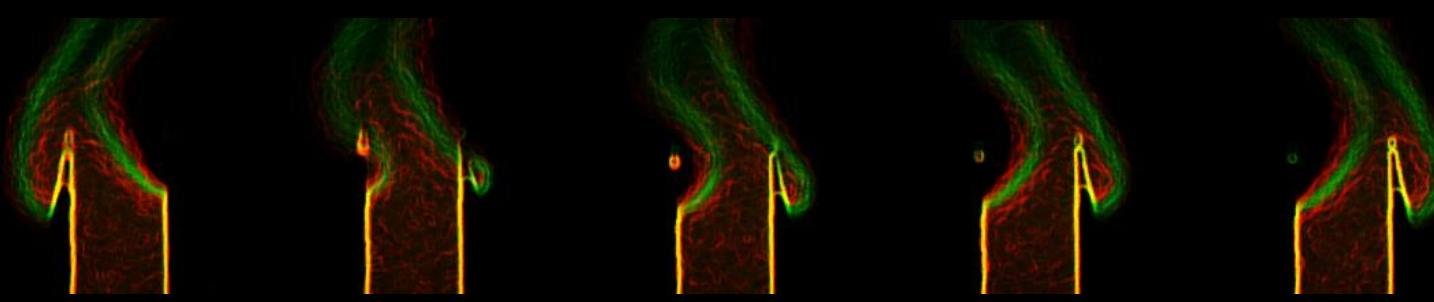

읍 


\section{DEVELOPING MICROFLUIDIC TOOLING FOR 3D CELL-CULTURE}

Joshua Taylor Loessberg-Zahl 
This dissertation has been approved by:

supervisors

Prof. Dr. Ir J.C.T. Eijkel

Prof. Dr. A. van den Berg

Cover design: J. T. Loessberg-Zahl

Printed by: IPKAMP printing

Lay-out: J. T. Loessberg-Zahl

ISBN: 978-90-365-4920-2

DOI: $10.3990 / 1.9789036549202$

(C) 2019 Joshua Taylor Loessberg-Zahl, The Netherlands. All rights reserved. No parts of this thesis may be reproduced, stored in a retrieval system or transmitted in any form or by any means without permission of the author. Alle rechten voorbehouden. Niets uit deze uitgave mag worden vermenigvuldigd, in enige vorm of op enige wijze, zonder voorafgaande schriftelijke toestemming van de auteur. 


\title{
DEVELOPING MICROFLUIDIC TOOLING FOR 3D CELL-CULTURE
}

\author{
DISSERTATION
}

$$
\text { to obtain }
$$

the degree of doctor at the Universiteit Twente, on the authority of the rector magnificus,

Prof.dr. T.T.M. Palstra,

on account of the decision of the graduation committee to be publicly defended

on Friday 20 December 2019 at 12.45 hrs

by

\author{
Joshua Taylor Loessberg-Zahl \\ Born on the $4^{\text {th }}$ of April 1992 \\ in Placerville, California, USA
}




\section{Graduation Committee:}

Chairman / secretary

Supervisors:

Other Supervisors:

Committee Members:
Prof. Dr. J.N. Kok

Prof. Dr. J.C.T. Eijkel

Prof. Dr. Ir. A. van den Berg

Dr. A.D. van der Meer

Prof. Dr. J.C.T. Eijkel

Prof. Dr. A. van den Berg

Dr. A.D. van der Meer

Prof. Dr. H.B.J. Karperien

Prof. Dr. S.J.G. Lemay

Prof. Dr. A.J. van Zonneveld

Dr. E. Delamarche 
If nothing is working,

and no one can tell you why:

You're probably on to something. 



\title{
Table of Contents
}

\author{
Introduction
}

References.

Chapter 1: Flow Patterned Wettability for Rapid Prototyping of 3D Cell

\section{Culture Geometry}

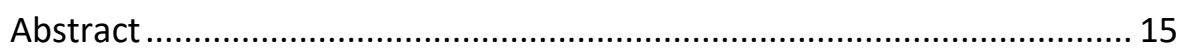

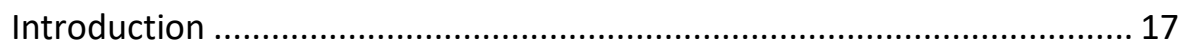

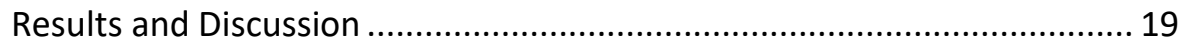

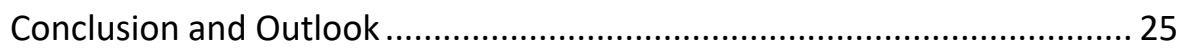

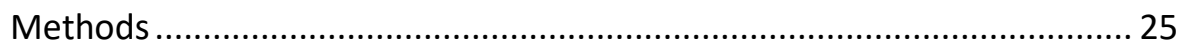

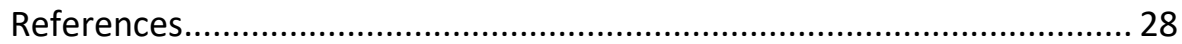

Chapter 2: Flow Focusing Through Gels as a Tool to Generate 3D

Concentration Profiles in Hydrogel-Filled Microfluidic Chips

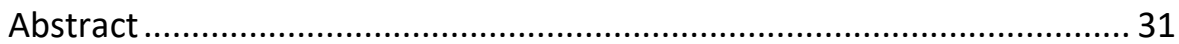

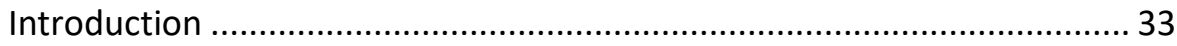

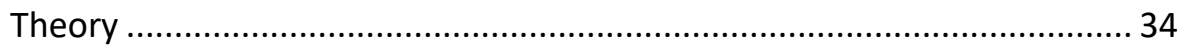

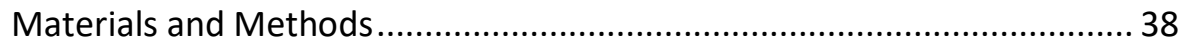

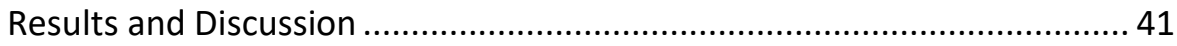

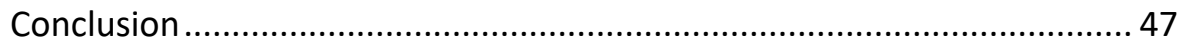

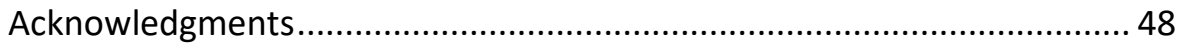

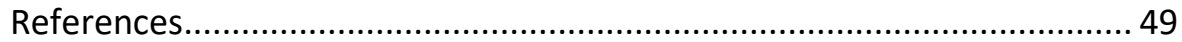

Chapter 3: Towards Precise Control of Microvascular Geometry in SelfOrganized Microvascular Networks

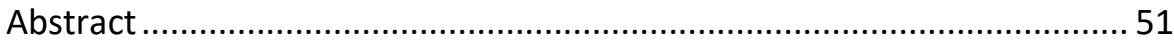

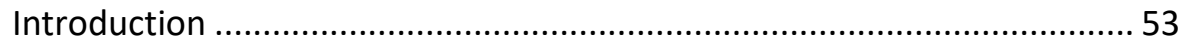

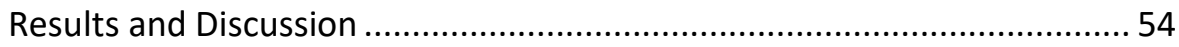

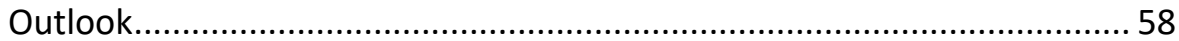




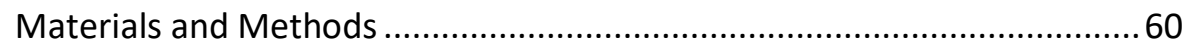

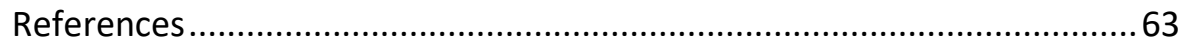

Chapter 4: Diffusion from Steady State Profile (DSSP) for Low Cost, Low

Concentration Characterization of Protein Diffusivity

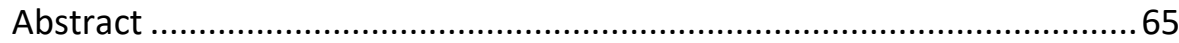

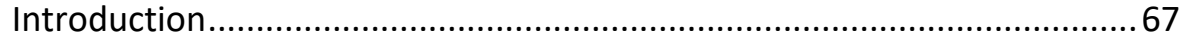

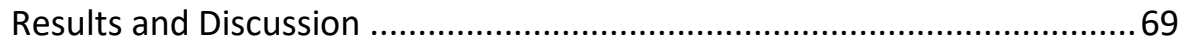

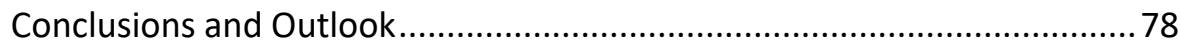

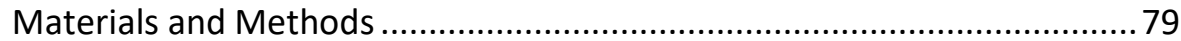

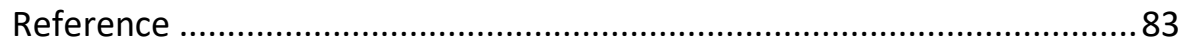

Chapter 5: Chip holder-integrated Pneumatic Logic for Modular Multiplexing of Microfluidic Devices

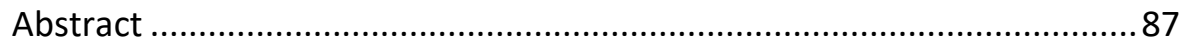

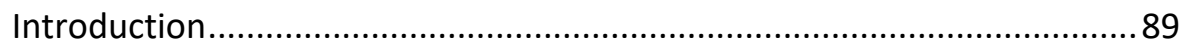

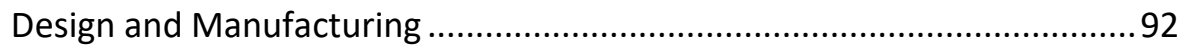

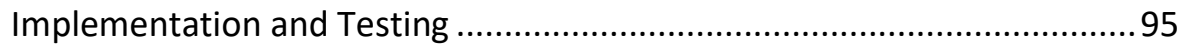

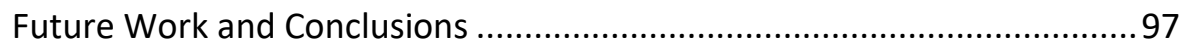

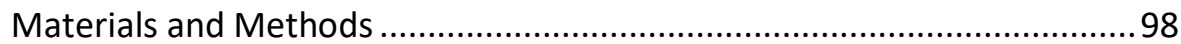

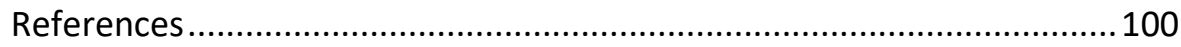

Chapter 6: Voltage Mediated Delamination of Suspended 2D Materials Explains Commonly Observed Breakdown

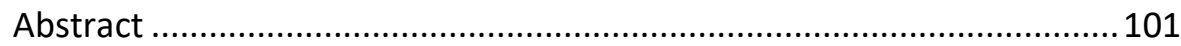

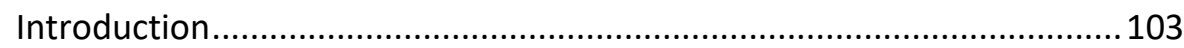

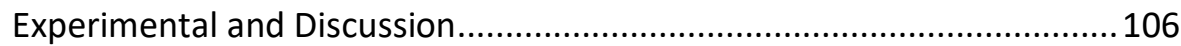

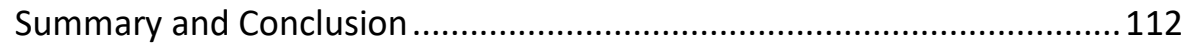

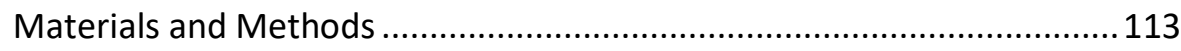

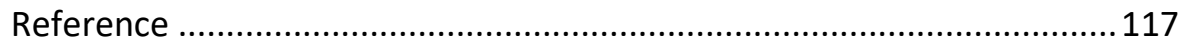




\section{Summary and Outlook}

\section{Appendices}

Appendix 1: Chapter 2 Supplementary Information................................ 131

Appendix 2:_Chapter 4 Supplementary Information................................. 141

Appendix 3:_Chapter 6 Supplementary Information................................. 149

\section{Publication List}

Thank You! 


\section{Introduction}

This thesis admittedly covers a broad range of topics. While our exploration over the last four years has taken us far afield, from developing microfluidic multiplexers to uncovering breakdown modes of $2 \mathrm{D}$ material systems, this work was funded by the Vascular Engineering on chip using differentiated Stem Cells (VESCEL) grant of Albert van den Berg and, as such, most chapters make significant steps towards the goal of creating 3D microvascular networks with custom geometry. The first three chapters in particular each build directly towards this goal, by introducing new techniques for the control of the microenvironment around human cells in 3D hydrogels.

Our work began with an improvement of the state-of-the-art platforms for making human microvasculature on chip. Existing techniques often relied on geometry like arrays of pillars or phase guides to confine hydrogels to specific regions within a microfluidic channel $[1,2,3]$ The technique that we developed to remove the need for any confining device geometry is detailed in chapter 1 . Gels in our devices were instead guided by patterns in the wettability of the surface of our channels. These patterns were easily changed without redesigning the device and reduced the contact area between cultured cells and the unnaturally stiff material of the devices. In spite of their relative lack of support, the patterned gels could withstand stresses from flow velocities far faster of those observed in the human body.

Next, we began to work towards a technique to locally apply signaling chemicals within a 3D hydrogel with the eventual goal of locally modifying a growing microvasculature network. Existing techniques for generating concentration landscapes within hydrogels predominantly rely on diffusion to move the chemicals to where they are needed.[4] However our own preliminary experiments and recent published work had shown that the resulting diffusive gradients were easily disrupted by flow through the hydrogel. $[5,6]$ We leveraged this observation to our advantage, using flow through the hydrogel to actively guide a stream of signaling chemicals to a desired location. In chapter $\mathbf{2}$ we detail this technique and demonstrate its ability to precisely and reliably generate concentration profiles in both $2 \mathrm{D}$ and 3D.

Finally, we began work to apply this technique to make on-chip microvascular networks with well-ordered geometry. Existing techniques for 
making blood vessels either create vessels which are much larger than the microvessels found in human tissues[1,7,8], or produce highly disordered networks. $[1,9,10]$ Our starting point was the disordered networks generating using a technique developed by the group of R. Kamm.[10] In chapter 3 we show our ability to use flow through the gel to guide simple gradients over these networks and show that our technique for generating concentration profiles is not disrupted by the on chip microvascular networks. This work is still in progress, and our next step will be to attempt actual modification of these networks by generating concentration profiles of various signaling chemicals over the networks. Eventually we hope to stimulate the growth of single vessels from this network and guide their growth, producing a smallscale vessel with precisely controlled geometry.

While some of the last three chapters are related to the goal of microvascular control, they each aim to solve relatively independent technical problems. Chapters 4 and 5 present solutions to general problems that were encountered while working with 3D cell cultures while Chapter 6 is a study of the physics behind a curious failure mode of nanopore-based sensors. Since the work in chapter 6 was performed in parallel with the other work during the $\mathrm{PhD}$, it is included in this thesis. Our brief introduction to each of these less related works follows below.

In chapter $\mathbf{4}$ we describe a technique to measure the diffusivity of fluorescently labeled proteins using tools common to the 3D cell culture community. This work was directly motivated by our need to know the diffusivity of the signaling chemicals we sought to guide in chapters 2 and 3 . The lack of a good repository of protein diffusivities or a quick and dirty method to measure them pushed us to develop our own technique. Our technique uses a combination of convection and diffusion to generate a steady-state concentration profile in a hydrogel similar to the profiles generated in chapters 2 and 3 . The form of the profile is then used to extract the diffusivity of the transported chemical. Our technique recovers similar information as existing techniques like FRAP but with less severe requirements on imaging equipment.

Lack of a good and general technique for multiplexing devices is an almost universal problem in microfluidics. To combat this, complex on-chip valve arrays are often used to multiplex devices. These are unfortunately difficult to fabricate, making them particularly cumbersome when the un- 
multiplexed device is relatively simple.[11,12] Less often, existing methods for multiplexing like pipetting robots are used, but these are expensive and not optimized for microfluidics.[3] In chapter 5 we develop in-chip holder multiplexers as a compromise especially designed for microfluidics. These would deliver the same functionality as on-chip multiplexers but move the complexity to a cheap and reusable chip holder, leaving the on-chip microfluidics simple. To do this we developed an improved, leak-free, clamped gasket valve. With our valves, small pressures can be used to switch relatively larger ones making them ideal logical elements. We demonstrate the efficacy of these valves and the potential of in-chip holder multiplexing by implementing and testing a pneumatic shift register built into a chip holder. In our next steps, we aim to show that our chip holders can be used to dispense liquid to a variety of simple microfluidic devices.

The final chapter is only loosely related to the other five as it explores an unexpected nanofluidic phenomenon. This chapter stemmed from previous work exploring the voltage driven transport of ions through single layer graphene as detailed in the Thesis of Wesley van den Beld. In those preceding experiments, we observed that when higher voltages than the typical ones were applied, the current through the system became highly nonlinear. While this behavior had been reported several times before $[13,14,15]$ most theories used to explain this behavior assumed that this breakdown of resistance was irreversible while the phenomenon we observed was strikingly reversible. This motivated our continued exploration which eventually yielded our theory that voltage-mediated delamination of the graphene from its substrate was the cause. The wide body of supporting evidence that we collected while exploring this phenomenon and the reasoning behind our theory is presented in chapter 6 .

At the foundation of these six chapters are the lessons we learned while striving and failing to answer deeper or sometimes wholly unrelated questions. We hope that in reading these chapters, other researchers will find a solution to a problem they face or the inspiration to make opportunity out of experiments gone awry. 


\section{References}

${ }^{1}$ H. Lee, M. Chung, N. L. Jeon, Materials Research Society Bulletin, 2014, 39, 51.

${ }^{2}$ C. Kim, J. Kasuya, J. Jeon, S. Chung, R. D. Kamm, Lab on a Chip, 2015, 15, 501.

${ }^{3}$ V. van Duinen, A. van en Heuvel, S. J. Trietsch, H. L. Lanz, J. M. van Glis, A. J. van

Zonneveld, P. Vulto, T. Hankemeier, Scientific Reports, 2017, 7, 18071.

${ }^{4}$ V. van Duiden, S. J. Trietsch, J. Joore, P. Vulto, T. Hankemeier, Current Oppinion in Biotechnology, 2015, 35, 118.

${ }^{5}$ V. S. Shirure , A. Lezia , A. Tao , L. Alonzo and S. C. George , Angiogenisis, 2017, 20 , 493

${ }^{6}$ R. Sudo, S. Chung , I. K. Zervontonakis, V. Vikerman, Y. Toshimitsu , L. G.

Griffith and R. D. Kamm, The Journal of the Federation of American Societies for Experimental Biology, 2009, 23, 2155.

${ }^{7}$ K. Haase, R. D. Kamm, Regenerative Medicine, 2017, 12, 285.

${ }^{8}$ P.F. Costa, H.J. Albers, J. E. A. Linssen, H. T. Middelkamp, L. van der Hout, R. Passier, A. van den Berg, J. Malda, A. D. van der Meer, Lab on a Chip, 2017, 17, 2785.

${ }^{9}$ J. A. Whisler, M. B. Chen, R. D. Kamm, Tissue Engineering Part C, 2014, 20, 543

${ }^{10}$ G. S. Offeddu, L. possenti, J. Loessberg-Zahl, P. Zunino, J. Roberts, X. Han, D.

Hickman, C. G. Knutson, R. D. Kamm, Small, 2019, 1902393.

11 J. Melin, S. R. Quake, Annual Review of Biophysics and Biomolecular Structure, 2007, 36, 213.

12 M. Mehling, S. Tay, Current Oppinion in Biotechnology, 2014, 25, 95.

${ }^{13}$ K.Liu, J. Feng, A. Kis and A. Radenovic, , ACS Nano, 2014, 8, 2504-2510.

${ }^{14} \mathrm{H}$. Cun et al., Centimeter-Sized Single-Orientation Monolayer Hexagonal Boron Nitride With or Without Nanovoids, Nano Lett. 18, 2018, 1205-1212.

${ }^{15}$ A.Esfandiar, B. Radha, F.C. Wang, Q. Yang, S. Hu, S. Garaj, R.R. Nair, A.K. Geim, K. Goopinadhan, Size effect in ion transport through angstrom-scale slits, Science, 358, 2017, 511-513. 



\title{
Chapter 1: Flow Patterned Wettability for Rapid Prototyping of 3D Cell Culture Geometry
}

Contributions by: Joshua T. Loessberg-Zahl, Jelle Beumer, Albert van den Berg, Andries van der Meer and Jan C. T. Eijkel

\begin{abstract}
Here we present a technique for facile patterning of hydrogel geometries commonly found in 3D cell culture literature, but without the need for any confining geometry built into the channel. Core to the technique is the use of laminar flow patterning to create a hydrophilic path through an otherwise hydrophobic microfluidic channel. When a liquid hydrogel is injected into the hydrophilic region, it is confined to this path by the surrounding hydrophobic regions. The various surface patterns possible via laminar flow patterning can thus be rendered into 3D hydrogel structures. We demonstrate that the technique can be used in many different channel geometries while still giving the user control of key geometric parameters of the final gel. Furthermore, the patterned gels are biocompatible and can withstand trans-gel pressures in excess of those needed to generate physiological flow velocities.
\end{abstract}




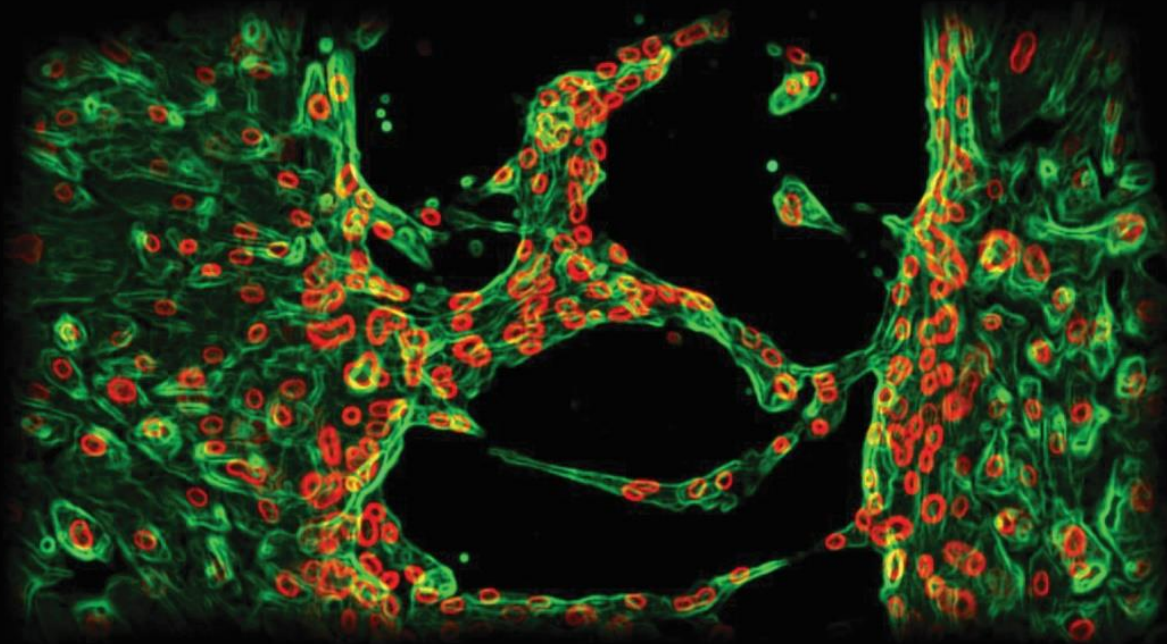




\section{Introduction}

Partially supported hydrogels are a common motif in microfluidic 3D cell culture. [1,2,3] Fragile hydrogels are often supported by the relatively rigid walls of some microfluidic enclosure on one or more sides, leaving the other sides accessible for cell seeding and nutrient delivery. One of the most common designs is a gel sandwiched between two fluidic access channels (Figure 1.1 a). $[4,5,6,7,8,9,10,11,12]$ This geometry in particular has found a wide range of applications as it allows both perfusion of the gel $[5,6,7]$ and chemical gradient generation $[8,9,10,11,12]$.

The geometry of the gel filled region is key in determining the culture conditions therein. When specific chemical gradients are required, the width of the gel directly determines the steepness of the gradient. Similarly, the width of the gel filled region often determines the flow resistance of the device the and thus the shear experienced by cells in the gel for a given applied transgel pressure. Therefore design flexibility in the gel geometry, particularly its width is highly desirable for precise definition of the cell culture conditions therein.

Current fabrication techniques for sandwiched gel devices require significant extra fabrication when the geometry of the gel needs to be changed. This is because the geometry of the gel filled region is often totally defined by the geometry of the surrounding device. Most commonly gel confinement is achieved via an array of pillars $[10,11]$, or sometimes phase guides[12,13]. In both cases, even small changes to the gel geometry require revision of these confining features. This takes extra time in the best case, but as devices are often molded from cleanroom processed wafers, redesign can often be quite expensive, requiring the procurement of new lithography masks and extra cleanroom time.

The features used to confine the gel can also influence cells cultured on the gels in undesirable ways. Cells types cultured in monolayers on the gelmedia interface are often known to have strong interactions with stiff and rough surfaces. $\left[{ }^{14},{ }^{15}\right]$ Proximity to protruding features on the surface of the microfluidic device used to keep the gel in place, particularly pillars, can therefore affect cell phenotype. When pillars are used to confine the gel, cells often have trouble bridging the gap between pillars and gel, and instead creep along the boundary between the two. This can make the monolayer unduly rough and in the worst case leave it leaky with large intrusions of the monolayer into the gel. $[9,10,11]$ Integrity of the monolayer is particularly important as the cell monolayer is often being studied directly or is included 

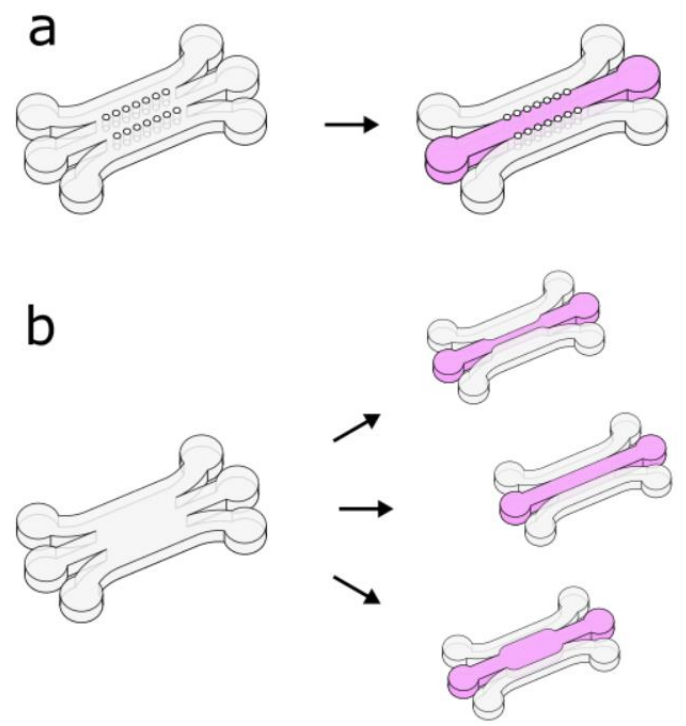

Figure 1.1: Schematic of 3-inlet, 3-outlet microfluidic devices where empty channels are shown in white and gel filled channels are shown in pink. (a) Typical device found in the literature where geometry of the gel filled region is totally determined by the geometry of the empty channel, in this case pillars. (b) Our devices which have no confining geometry. The width of the gel region can be changed without the need to change the design of the starting device.

to ensure that physiological levels of nutrients or growth factors reach cells in the bulk gel $[1,2,3]$.

The technique we present here allows the creation of a gel filled region between two fluidic access channels, without the need for any confining geometry built into the microfluidic device. This is achieved via laminar flow patterning of the wettability of a standard 3-inlet, 3-outlet microfluidic device (Figure 1.1 bottom). Similarly to other techniques where surface wettability is patterned, a hydrophilic path is patterned through an otherwise hydrophobic device. $[16,17,18]$ When a hydrogel of choice is injected into the device, it stays confined to this hydrophilic path while it cures.

We show that the technique allows the easy adjustment of the width of the gel-filled region via adjustment of flow rates during patterning. No change to the surrounding channel geometry is required. Furthermore we demonstrate the technique in a number of different device geometries showing that strongly tapered can be made as well as long $(2 \mathrm{~cm})$ meandering 
gels. Finally we demonstrate successful culture of endothelial monolayers on the gels and show that the gels can withstand stresses far in excess of physiological conditions without being damaged in spite of the lack of confining features.

\section{Results and Discussion}

Here we will first explain how the hydrophillic path for the gel to follow is created and the reasoning behind critical steps in that procedure before demonstrating some capabilities of the technique.

\section{Implementation}

Surface chemistry is critical to this technique. The chemistry detailed here assumes that the user us working with PDMS or glass devices, although alternative chemistries do exist for plastics $\left[{ }^{19},{ }^{20}\right]$. The chemistry achieves two goals, first it ensures that regions of the device can be rendered robustly hydrophilic or hydrophobic and second, it ensures strong adhesion between the used hydrogel and the walls of the channel.

In our technique both hydrophobicity and strong protein adhesion are ensured by sequential surface treatments with (3-Aminopropyl)triethoxysilane (APTES) and glutaraldehyde (GA). If any protein with a free primary amine is introduced to the activated surface, it will become bound.

This surface treatment is inspired by literature, but heavily adapted to our application for ease of use. Similar surface treatments are commonly used in some protein-based sensor technologies, however they have stringent requirements on the thickness of the adsorbed layer of APTES and GA as it can affect the performance of the sensor. [21] As such, they are required to grow their layers slowly, often with cumbersome vapor deposition or organic solvent based techniques. We are not so limited, as all we require is that the surface is robustly hydrophobic and has many sites capable of binding proteins. Therefore our protocol differs from the literature protocols. Specifically, all surface treatments in our protocol are done in aqueous phase for ease of use and at relatively high contrition, reducing the reaction time from hours to minutes. See the methods section for further details

In the surface treatment protocol the surface is rendered hydrophobic and is ready for the patterning of hydrophilic regions. These hydrophilic regions are created by simply introducing a low concentration $(10 \mu \mathrm{g} / \mathrm{mL})$ solution of collagen in 1xPBS to regions desired to be hydrophilic. The collagen 
quickly (10 minutes) and covalently binds to the surface and, even after thorough drying and rinsing, render the surface robustly hydrophilic everywhere where it had contact.

To control the region of contact, laminar flow patterning is employed.[22] (figure 1.2) In the cases shown here, flow is driven through the three inlets on one side on the main channel by a syringe pump and allowed to flow out the three outlets on the other side. The middle stream, artificially dyed red with food coloring in figure 1.2, contains the coating solution. Flows are picked such that diffusion is small compared to the residency time in the device. This means that the collagen introduced in the middle stream stays wherever the middle stream is guided. Flow is maintained for $10 \mathrm{~min}$, before the channel is blown dry.

After flow patterning, the top and bottom of the main channel have been rendered hydrophilic while the rest of the device remains hydrophobic. Through the middle inlet, an uncured hydrogel (in this case $4 \mathrm{mg} / \mathrm{mL}$ collagen I) is introduced. While the liquid gel is free to easily wet the hydrophilic regions
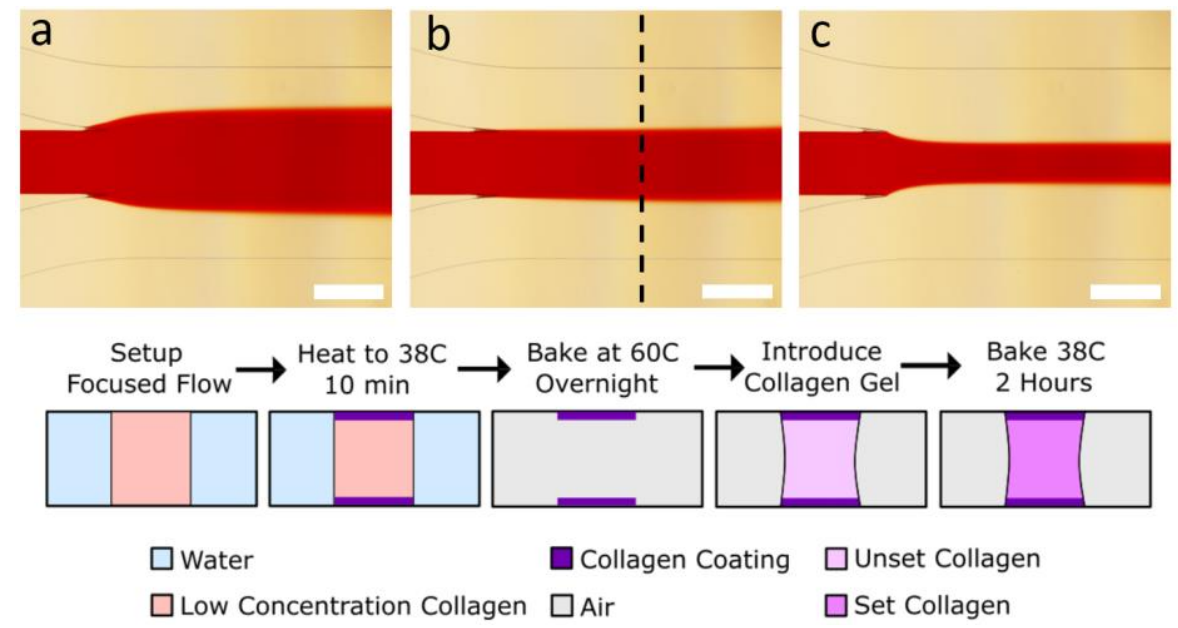

Figure 1.2: (top) example of laminar flow patterning in our devices. Flow is introduced via the 3 inlets on the left of each image, a red tracer dye is included with low concentration collagen in the central stream. Flow ratios, reported as top : middle : bottom were (a) 1:2.5:1, (b) 1:1:1, (c) 1:0.5:1. Scale bars are 500 microns. (bottom) Schematic of the coating process at a cross section taken at the dotted line shown in panel $b$. 
of the device, it pins on the edge between the hydrophilic and hydrophobic regions. After gel filling, the devices can be incubated to cure the gel. The fluidic access channels can filled with cell media and cell culture can begin.

In the end, the device contains a cured gel, confined to the same region of the chip as the coating solution was during laminar flow patterning. Through this technique, many of the surface patterns attainable via laminar flow patterning can be rendered in 3D gel. Going forward we demonstrate some of the possible patterns as well as our ability to fine tune the geometry of a few particular patterns without redesigning the device.

\section{Channel Geometry}

We patterned gels in three different types of microfluidic device to show the ability of our technique to create both typical gel geometries and new, potentially useful gel geometries. (Figure 1.3)

In the simplest case (figure 1.3a) we show that our technique can realize the typical sandwiched gel design often used for generating simple gradients over cell cultures in the literature. $[8,9,10,11,12]$. Similarly, in figure $1.3 \mathrm{~b}$ we show that our technique can capture geometries typically used to generate many different gradients in the same device [23].

In Figure $1.3 \mathrm{c}$ we show our ability to fabricate long, highly curved structures. The meandering channel shown in figure $1.3 \mathrm{c}$ is $2 \mathrm{~cm}$ in length and the patterned gel maintains an effective barrier between the two fluidic access channels for the entire length of the device. The curvature here is dictated by both the device geometry and the relative flowrates used for patterning.

The smooth interface shown here is difficult to achieve in the commonly used pillared devices, where the gel-media interface arcs from pillar to pillar $[10,11]$. However there are still some design constraints. In particular, the gel can only follow paths patternable via laminar flow patterning. Drawbacks of laminar flow patterning, like the propensity of flow to cut tight corners can cause some distortion of the patterned geometry (Figure 1.3c) and must therefore be accounted for.

In the three shown cases, we also have cultured hUVECs in the channels to highlight the biocompatibility of the technique. Notice that the hUVECs exhibit their healthy cobble-stone phenotype and remain attached to the substrate in all cases even after 5 days of culture. The patterned 

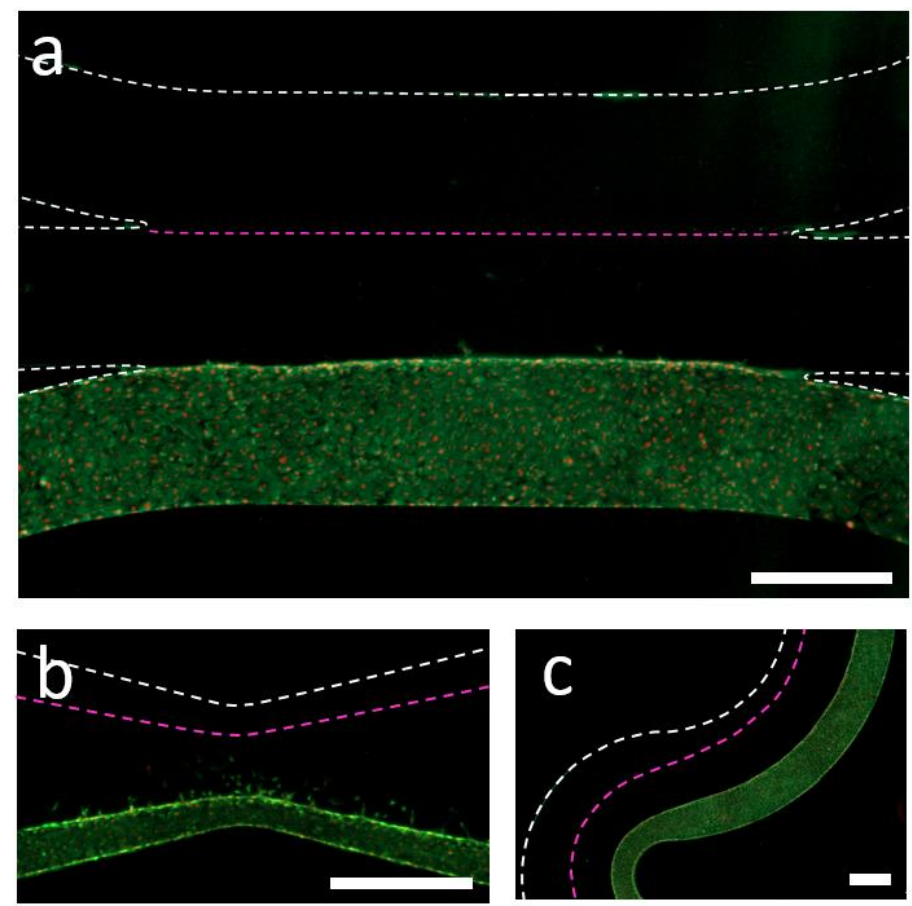

Figure 1.3: Sample of the variety of channel geometries that can be patterned by our technique. In each device, hUVECs have been cultured in one of the fluidic access channel. Actin is stained green and nuclei are stained red. The boundary between the gel and the other fluidic access channel is indicated by the dotted pink lines and the dotted white lines indicate the walls of the microfluidic channel. (a) shows the typical sandwiched gel design often used in the literature. (b) shows an hourglass shaped device which might be used for grating a range of gradients in a single device. (c) shows a meandering channel which demonstrates our ability to create curved cell-gel interfaces and long gel regions $(2 \mathrm{~cm})$. Scale bars are 500 microns.

geometries have also remained stable over the 5 days, indicating that the gel has similarly remained strongly bound to the walls.

\section{Width Control}

To control the width of the final gel, the flowrate of the central stream was varied during patterning while the flowrate of the side streams was left the same. (Figure 1.4) We demonstrate this in two different types of devises, a simple straight channel (Figure 1.4, top) and an hourglass shaped channel (figure 1.4 bottom). In these devices the fraction of the total channel width 
patterned can be estimated simply as the ratio of the patterning stream's flowrate to the total flowrate. [24]

In the case of the tapered channels, the gel filled region is similarly tapered as the collagen containing stream is forced to taper during patterning. Notice that both the angle of the taper and the fraction of the channel width taken up by the gel are both simultaneously changed by adjusting the flowrates. The minimum width of gel that we could reliably generate at the neck of the taper was 200 microns (figure 1.4, bottom right). Smaller widths resulted in the gel bursting out of the patterned region and filling the fluidic access channels. Thinner gels regions may be attainable with gentler filling or by using surface treatments with a higher contrast in hydrophilicity.
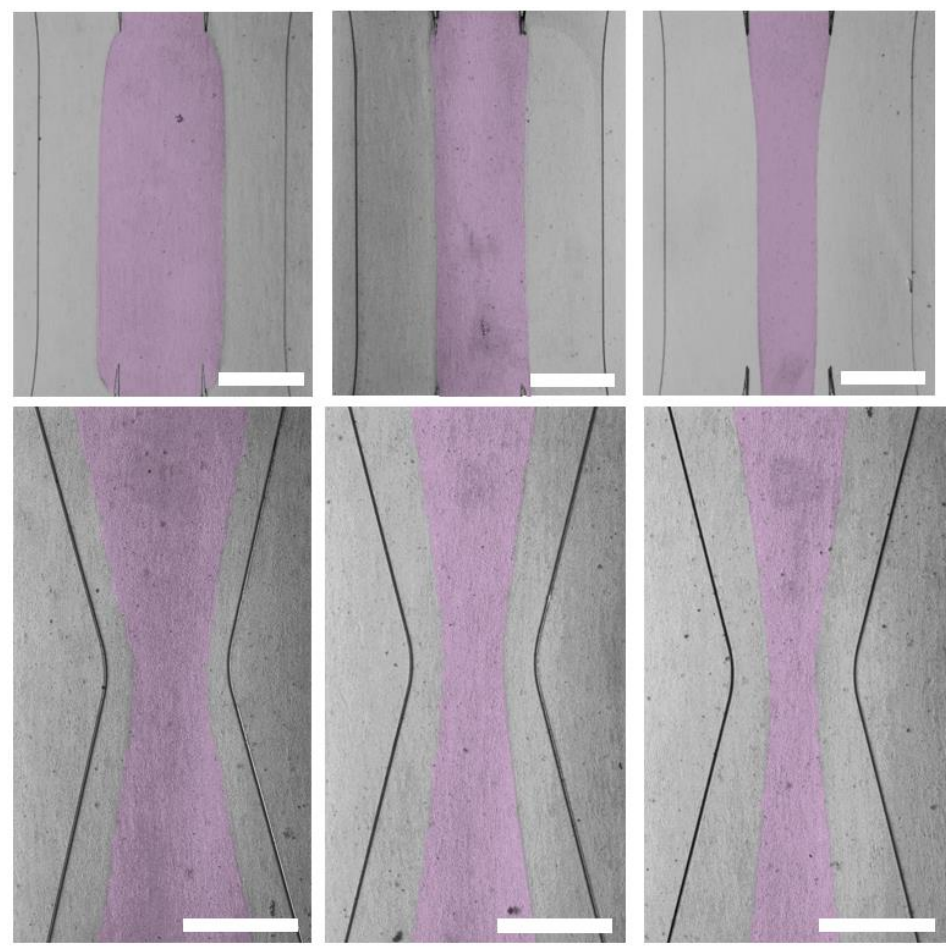

Figure 1.4: Demonstration of width control. Gel filled regions are artificially colored pink to guide the eye. Side channels are filled with PBS. Relative flow of the central stream decreases from picture to picture from left to right. Top row shows the typical straight channel sandwiched gel design, while the lower row shows our hourglass design. In the hourglass design, both the width of the gel region and the angle of taper are affected by the change in flowrate. 


\section{Interstitial Flow}

As both a stress test of our technique and a further proof of biocompatibility, we run a model experiment to measure the response of cell migration to shear stress in the gel. hUVECs were used in our proof of concept as they are known to migrate in the upstream direction when interstitial flow is applied[5,6,7]. We culture hUVECs in one fluidic access channel just as we did in figure 1.3. This time, a hydrostatic pressure was applied to the cell-free channel to induce trans-gel flow one day after seeding. While the flows we applied were several times higher than those expected under physiological conditions ( $50 \mu \mathrm{m} / \mathrm{s}$ ), an effect on the cell migration was still apparent.

Cell migration in response to flow is shown in figure 1.5 with quantified results shown in the right hand panel. The number of cells that had migrated from the seeded channel into the gel region were counted each day. A significant increase in the number of cells in the gel was observed as well as a reduction in the spread of the data. (Figure 1.5 right)

The gels also showed no sign of collapse in spite of the fact that flow was significantly in excess of the physiological range. The stress on the gel and therefore damage to the gel is expected to increase with extra flow [25]. The
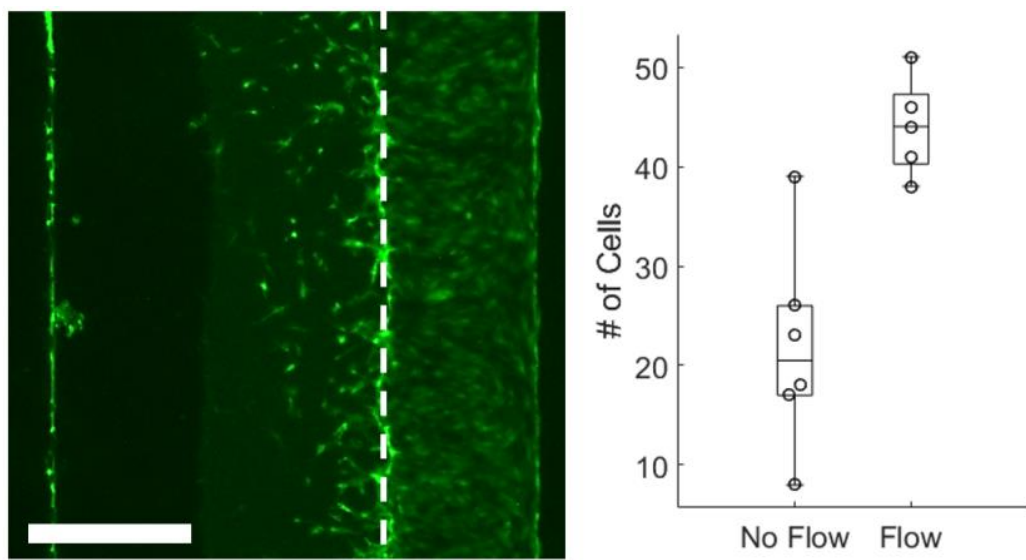

Figure 1.5: Right panel shows a sample device used for cell migration quantification. The dotted line indicates the gel-media interface. Cells to the left of the dotted line were considered to have migrated into the gel and were counted. Scale bar is $\mathbf{5 0 0}$ microns. Left panel shows the number of cells counted in the gel on day 4 . The addition of flow appears to both increase the count and reduce the spread in the data. 
fact that no damage was seen at these flowrates implies that experiments executed within the physiological range of flowrates will leave the gel similarly unscathed.

\section{Conclusion and Outlook}

We have designed and tested a new technique for making gel structures for 3D cell culture in microfluidic devices. Our technique can reproduce the most commonly used gel structures, but differs from existing techniques in that it uses laminar flow patterning to define the gel geometry instead of confining structures like pillars or phase guides. This both allows rapid prototyping of the gel geometry and reduces contact between cells and the unnaturally stiff walls of the device.

We have shown that the technique is usable in a variety of geometries, both common in the literature and novel. This includes devices with gels far longer and with higher curvature than is commonly needed. Width of the gel is easily controlled by adjusting flow parameters during patterning. Once gels are patterned and cured, cells seeded in these devices grow as expected. Finally, we show that devices can withstand trans-gel pressures far in excess of what is needed to reproduce physiological conditions. Taken together, the features provided by this technique should make it a powerful tool for fast implementation and iteration of 3D cell culture devices.

\section{Methods}

\section{PDMS Device Fabrication}

To fabricate the PDMS devices, un-cured PDMS was cast on an SU8 on silicon mold. SU8 features were 100 micrometers high. Un-cured PDMS (Sylgard) was prepared at a 10:1 polymer to cross linker ratio. The devices were then heated overnight at $60^{\circ} \mathrm{C}$ to cure the polymer before demolding. In parallel, microscope slides dipped in the same PDMS were prepared as a substrate for bonding. Coated slides were similarly cured overnight at $60^{\circ} \mathrm{C}$. After demolding, inlets were punched using a $1 \mathrm{~mm}$ biopsy punch. Both the cast devices and PDMS coated slides were then exposed to oxygen plasma in a plasma cleaner (Harrick) to activate their surfaces. The cast polymer was then gently pressed onto the PDMS coated slides to form the completed PDMS devices. 


\section{Surface Treatment}

To make the surface able to covalently bind proteins, sequential (3Aminopropyl)triethoxysilane (APTES) and glutaraldehyde (GA) treatments were performed. This protocol was applied within 15 min after the plasma treatment described in the previous step. A 3\% (v/v) solution of APTES (SigmaAldrich) in deionized water was first introduced into the devices and left to sit for $30 \mathrm{~min}$. Filtered air was blow through the devices to dry them and the devices were flushed three times with deionized water. A $10 \%(\mathrm{v} / \mathrm{v})$ solution of GA (Sigma-Aldrich) was then prepared in 1x phosphate buffered saline (PBS) (Sigma-Aldrich). This solution was similarly pipetted into the devices and allowed to sit for $30 \mathrm{~min}$. Finally the devices were again blown dry and rinsed three times with deionized water. Devices were then baked at $60^{\circ} \mathrm{C}$ overnight to drive off any excess water or reactants.

\section{Flow Patterning}

To pattern the devices, a 10 micro gram per milliliter solution of collagen was first prepared. This was done by gently mixing rat tail collagen I (Corning) with cold $1 x$ PBS. The solution was then loaded into a $1 \mathrm{~mL}$ syringe (Hamilton) and placed in a syringe pump (neMYSES) along with two deionized water containing $1 \mathrm{~mL}$ syringes. Tygon tubing (TYGON) was used to connect the syringes to the device. The middle inlet was connected to the collagen containing syringe while the two outside channels were connected to the deionized water containing syringes. Tygon tubing was also used to connect the outlets to a waste container. Tubing from the middle outlet was cut slightly shorter to ensure that the entire stream of coating solution flowed out this outlet. Finally the pumps were used to drive flow through the devices with flowrates of 30 microliters per min each. The flowrate of deionized water streams were left constant while the flowrate of the collagen containing stream was adjusted to change the width of the patterned region depending on the desired gel region width. Flow was maintained for $10 \mathrm{~min}$ for complete patterning. Devices were then blown dry using filtered compressed air and baked overnight at $60^{\circ} \mathrm{C}$ to dry completely.

\section{Filling and Cell Seeding}

A $4 \mathrm{mg} / \mathrm{mL}$ solution of rat tail collagen I was prepared at neutral $\mathrm{pH}$ on ice. This solution was gently pipetted into the central inlet of the patterned microfluidic devices and cured for 2 hours at $37^{\circ} \mathrm{C}$. To fill the fluidic access channels, devices were submerged in degassed 1xPBS overnight. The next day, 
the fluidic access channels are filled with cell media and seeded with cells of choice. In this case EGM (Sigma-Aldrich) and hUVECS (Lonza) were used. Cell media was replaced every day during culture.

\section{Interstitial Flow Experiments}

Devices were first seeded as described above. Flow through the gel was applied one day after seeding. In these experiments, the inlets were fitted with pipette tips as reservoirs. Reservoirs on the gel inlet and outlet were filled with PDMS to prevent outflow of media. Reservoirs connected to fluidic access channels were filled with cell media. Reservoirs connected to the cell containing fluidic access channel of the device were filled with less media than reservoirs on the other inlets leaving a height difference of $\sim 1.5 \mathrm{~cm}$. This height difference was refreshed every day when media was replaced. 


\section{References}

${ }^{1}$ V. van Duiden, S. J. Trietsch, J. Joore, P. Vulto, T. Hankemeier, Current Oppinion in Biotechnology, 2015, 35, 118.

${ }^{2}$ N. Kashaninejad, M. R. Nikmaneshi, H. Moghadas, A. K. Oskouei, M. Rismanian, M. Barisam, M. Saidi, B. Firoozabadi, micromacines, 2016, 7, 130.

${ }^{3}$ A. Hasan, A. Paul, N. E. Vrana, X. Zhao, A. Memic, Y. Hwang, M. R. Dokmeki. A. Khademhosseini, Biomaterials, 2014, 35, 7308.

${ }^{4}$ A. Hasan, A. Paul, N. E. Vrana, X. Zhao, A. Memic, Y. Hwang, M. R. Dokmeki. A. Khademhosseini, Biomaterials, 2014, 35, 7308.

${ }^{5}$ W. J. Polacheck , J. L. Charest and R. D. Kamm, Proc. Natl. Acad. Sci. U. S. A., 2011, $108,11115$.

${ }^{6}$ V. S. Shirure , A. Lezia , A. Tao , L. Alonzo and S. C. George , Angiogenisis, 2017, 20 , 493

${ }^{7}$ R. Sudo, S. Chung, I. K. Zervontonakis, V. Vikerman, Y. Toshimitsu , L. G.

Griffith and R. D. Kamm , FASEB J., 2009, 23, 2155

${ }^{8}$ S. Bersini, J.S. Jeon, D. Dubini, C. Arrigoni, S. Chung, J. L. Charest, M. Moretti, R.D. Kamm, Biomaterials, 2014, 35, 2454.

${ }^{9}$ G. S. Jeong, S. Han, Y. Shin, G. H. Kwon, R.D. Kamm, S. Lee, S. Chung, Analytical Chemistry, 2011, 83, 8454.

${ }^{10}$ C. Kim, J. Kasuya, J. Jeon, S. Chung, R. D. Kamm, Lab on a Chip, 2015, 15, 501.

${ }^{11}$ S. Kim, H. Lee, M. Chung, N. L. Jeon, Lab on a chip, 2013, 13, 1489.

${ }^{12}$ S. J.Trietsch, G. D. Israels, J. Joore, T. Hankemeier, P. Vulto, Lab on a Chip, 2013, 13, 3548.

${ }^{13}$ P. Vulto, S. Podszun, P. Meyer, C. Hermann, A. Manz, G. A. Urban, Lab on a Chip, 2010, 11, 1561.

${ }^{14}$ M. Lampin, R. Warocquier-Clerout, C. Legris, M. Degrange, M. F. Sigot-Luizard, Journal of Medical Materials Research, 1998, 36, 99.

${ }^{15}$ A. Pathak, S. Kumar, Proceedings of the National Academy of Science, 2011, 109, 10334.

${ }^{16}$ B. Zhao, J. S. Moore, D. J. Beebe, Scientific Reports, 2001, 291, 1023.

${ }^{17}$ A. Aota, M. Nonaka, A. Hibara, T. Kitamori, Angewandte Chemie International Edition, 2007, 46, 878.

${ }^{18}$ A. Hibara, S. Iwayama, S. Matsuoka, M. Ueno, Y. Kikutani, M. Tokeshi, T. Kitamori, Analytical Chemistry, 2005, 77, 943.

${ }^{19}$ E.A. Klassen, A. Rigutti, A. Bos, L.F. Bernini, Journal of immunological Methods, 1983, 59, 181.

${ }^{20}$ R. Khnouf, D. Karasneh, B. A. Albiss, Electrophoresis, 2015, 37, 529.

${ }^{21}$ N. S. G. Gunda, M. Singh, L. Norman, K. Kaur, S. K. Mitra, Applied Surface Science, 2014, 305, 522.

22 P. J. A. Kenis, R. F. Ismagilov, G. M. Whitesides, Science, 1999, 285, 88.

${ }^{23}$ B. M. Baker, B. Trappmann, S. C. Stapleton, E. Toro, C. S. Chen, Lab on a Chip, 2013,13, 3240. 
${ }^{24}$ R. F. Ismagolov, A. D. Stroock, P. J. A. Kenis, G. Whitesides, H. A. Stone, Applied Physics Letters, 1999, 76, 2376.

25 P. L. Chandran, V. H. Barocas, Journal of Biomechanical Engineering, 2004, 126, 152. 



\title{
Chapter 2: Flow Focusing Through Gels as a Tool to Generate 3D Concentration Profiles in Hydrogel-Filled Microfluidic Chips ${ }^{1}$
}

\author{
Contributions by: Joshua Loessberg-Zahl, Andries D. van der Meer, \\ Albert van den Berg, and Jan C. T. Eijkel
}

\begin{abstract}
Laminar flow patterning is an iconic microfluidic technology used to deliver chemicals to specific regions on a two-dimensional surface with high spatial fidelity. Here we present a novel extension of this technology using Darcy flow within a three-dimensional (3D) hydrogel. Our test device is a simple 3-inlet microfluidic channel, totally filled with collagen, a cured biological hydrogel, where the concentration profiles of solutes are manipulated via the inlet pressures. This method allows solutes to be delivered with 50 micron accuracy within the gel, as we evidence by controlling concentration profiles of $40 \mathrm{kDa}$ and $1 \mathrm{kDa}$ fluorescent polysaccharide dyes. Furthermore, we design and test a 3D-printed version of our device with an extra two inlets for control of the vertical position of the concentration profile, demonstrating that this method is easily extensible to control of the concentration profile in 3D.
\end{abstract}

${ }^{1}$ Adapted from:

J. Loessberg-Zahl, A. D. Van der Meer, A. van den Berg, J. C. T. Eijkel, Flow Focusing Through Gels as a Tool to Generate Concentration Profiles in Hydrogel-Filled Microfluidic Chips, Lab on a Chip, 2019, 19, 207. 


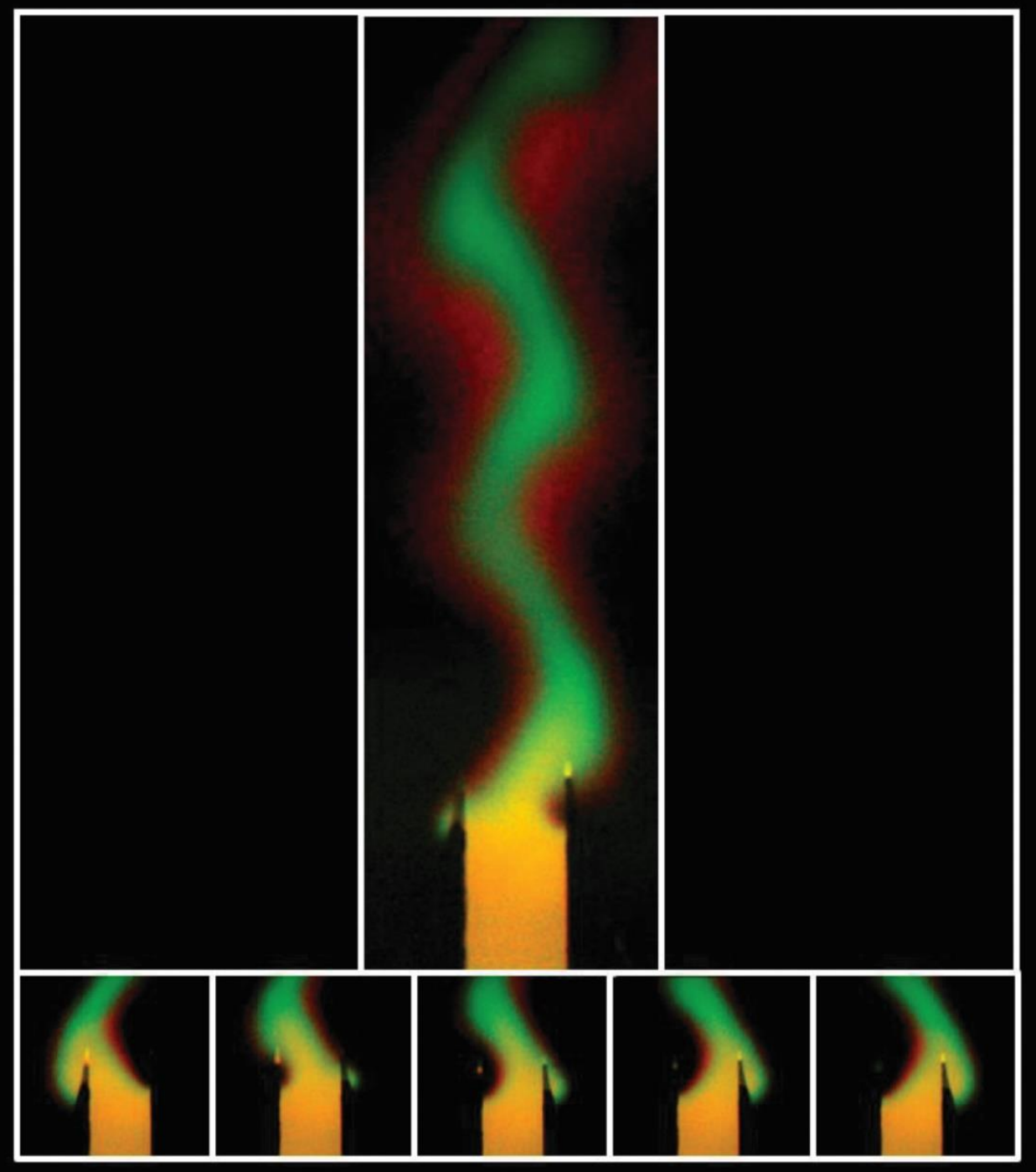




\section{Introduction}

While laminar flow patterning has been convincingly demonstrated as a surface patterning technique in microfluidic chips, $[1,2]$ an extension to patterning of three-dimensional (3D) biological matrices initially seems counterintuitive. Gels are often used in microfluidics for their ability to prevent flow while allowing transport of chemicals via other mechanisms such as diffusion[3] or electrophoresis. [4] However many biological hydrogels such as collagen have been shown to allow substantial amounts of flow while significantly limiting diffusive transport.[5] Furthermore, pressure-driven flows through these gels exhibit a plug flow profile, which simplifies flow control when compared to the parabolic flow profiles common to laminar flow patterning in liquid-filled channels.[6] These unique properties of biological hydrogels make them an ideal medium for laminar flow patterning.

Hydrogels are also a highly useful medium for microfluidics in general and in the past decades a number of microfluidic techniques have been developed that depend on the use of hydrogels.[7] To facilitate such techniques, patterning of concentration, composition, and geometry of hydrogels has been explored for a broad range of applications.[8] Local functionalities such as photodegradability have been added to make gel properties dynamically tunable for cell culture.[16] Biological gels have been patterned with either soft lithography or capillary barriers to make perfusable microfluidic devices.[17-20] Ion patterning of actuatable hydrogels has been used to implement soft robots.[21] Degradable subunits have been locally added to gels to affect timed release of drugs.[22] Here we present a patterning technique that allows accurate and dynamic control of concentration profiles in a gel, adding a new precisely controllable technique to the list.

In addition to serving as a general microfluidic tool for gel patterning, we find the potential cell culture applications particularly exciting. Flows through biological gels are a topic of prolonged interest in the context of onchip cell culture and organs-on-chips. $[40,41,42]$ Flows through the interstitial extracellular matrix are critical for a wide variety of biological processes from waste removal to embryogenesis to tumor development.[9-13] Recently, onchip platforms have been developed to study the influence of interstitial flow (IF) on cell behavior and have recapitulated its influence on, for example: cancer cell migration, ${ }^{14}$ alignment of smooth muscle cells, ${ }^{15}$ and 
lymphangiogenesis. However, the particular combination of solute gradients and IF has not been well studied in vitro even though inhomogeneity in the morphogen concentration is often critical and coincident with IF. [9,33] In fact, current techniques used to generate one condition almost always exclude the other.[38]

Our technique allows for independent control of both concentration profile and flowrate in a 3D hydrogel matrix. The technique is an analogue of laminar flow patterning and affords a similar degree of control for the position and shape of regions of different chemical solute composition.[1] As is the case of laminar flow patterning, we expect our technique to be widely applied for both local gel patterning and cell culture. We demonstrate the effectiveness of the technique with a physiologically relevant interstitial velocity of $10 \mu \mathrm{m} / \mathrm{s}$, within the range often used in similar devices,[14,23] with a $40 \mathrm{kDa}$ tracer dye chosen to match the diffusivity of the commonly used morphogen vascular endothelial growth factor (VEGF-A).[24] Furthermore, we demonstrate that the hydrodynamic time response of the system is fast compared to relevant biological timescales and that the technique is extensible to a fully $3 \mathrm{D}$ flow patterning setup for both horizontal and vertical control of the concentration profile.

\section{Theory}

In order to use flows through a gel as a tool to manipulate concentration profiles we must understand both how to effectively control flows through gels and how concentration profiles in a gel will evolve due to diffusion.

\section{Conceptual Description of Flow Model}

First, we developed a simple model to describe the dynamics of coflowing streams in a gel-filled microfluidic chip. Some adjustment from models based on laminar flow is required as flows through gel-filled channels have plug flow profiles[6] as opposed to the typical parabolic flow profile in microchannels. Accounting for plug flow actually simplifies the typical calculations and also implies other benefits such as reduced diffusion of large molecules[25] and reduced dispersion.[26,27]

Figure 2.1 schematically depicts the system, which consists of a microfluidic channel with three inlets, entirely filled with a 3D hydrogel. We wish to determine the width and lateral position of the central stream in the 
main channel (green in the figure) as a function of the pressures applied to the three inlets. The behavior of the streams in the main channel depends on the relative contribution of each inlet flow to the total flow in the main channel. For example, if the flow through one inlet increases, then the width of its stream in the main channel will increase while the other streams will become narrower. Therefore to model the streams in the main channel we must first determine the flow rates through each inlet channel. Since our active controls

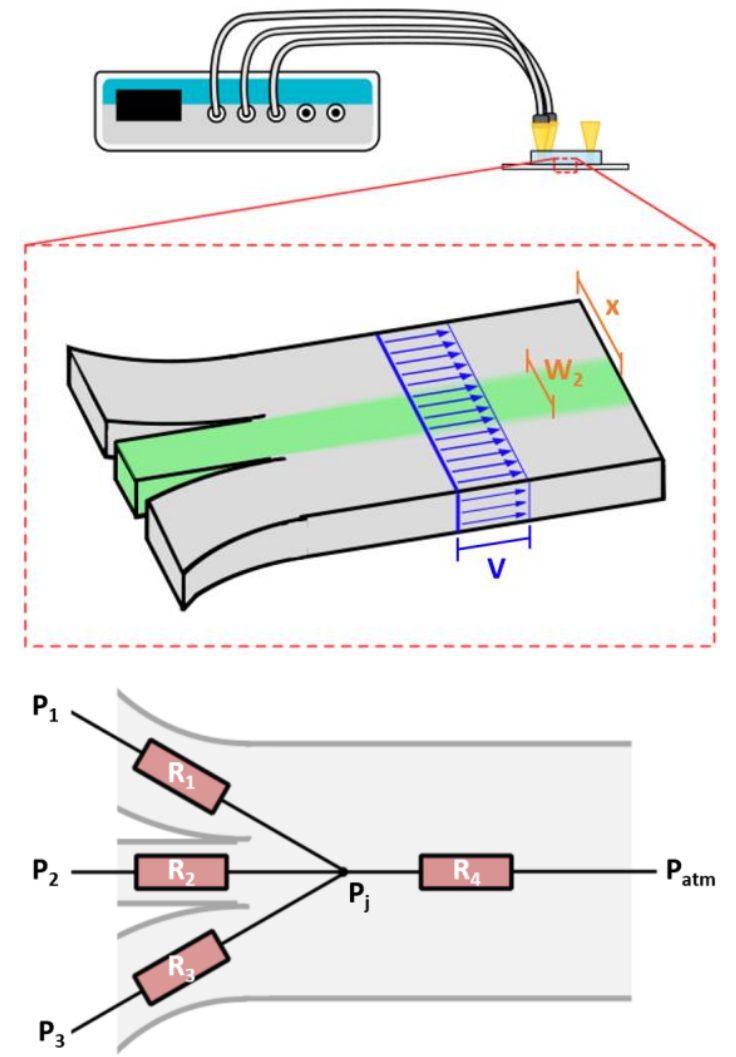

Figure 2.1: Flow in a microfluidic channel with multiple inlets connected to a pressure controller. Top, Schematic of 2D device and setup showing the plug flow profile in blue, and the two geometric constraints described by equations 2.5 and 2.6 in brown. Bottom, the resistor network used to model the system. Three inlet resistances feed into the junction and the resistance of the main channel connects the junction back to atmospheric pressure. Here our flow is through a porous media so we use the resistance as determined by Darcy's law as opposed to the typical Poiseuille resistance. 
are pressures, we begin by calculating the pressure-to-flow relation of the inlet channels.

\section{Darcy Flow}

In order to calculate flows in terms of pressures we calculate the hydraulic resistance of the system. As stated above, all flows in our chip take place in a porous medium, so modifications to the typical flow resistance equations must be made. The textbook definition of the flow resistance for a small, non-gel-filled, channel is:

\section{$2.1 \quad R_{\text {Poiseuille }}=\frac{8 \mu L}{A r^{2}}$}

Where $\mu$ is the viscosity, $L$ is the length of the channel, $A$ is the cross-sectional area of the channel and $r$ is the hydraulic radius of the channel. However in flows through porous media, the flow profile is instead given by Darcy's Law[6]

$$
2.2 v=\frac{\kappa}{\mu} \nabla P
$$

Where $\kappa$ is the permeability, $\nabla P$ is the local pressure gradient and $v$ is the local flow velocity. Note that the velocity has no dependence on spatial coordinates and is therefore uniform, implying a plug flow profile. Using equation 2.2 and our knowledge that flow resistance is simply the ratio between pressure drop and flow rate for a given geometry, we write the flow resistance for a gel-filled channel of length $L$ and uniform cross-section $A$ as:

\section{$2.3 \quad R_{\text {Darcy }}=\frac{\mu L}{\kappa A}$}

With the correct flow resistance for a gel in mind, we re-cast our system as the hydraulic resistor network shown in figure 2.1. In this system the flow rate in a given channel can be written in terms of the inlet pressures as:

$2.4 \quad Q_{n}=\frac{P_{n}}{R_{n}}-\frac{\frac{P_{1}}{R_{1}}+\frac{P_{2}}{R_{2}}+\frac{P_{3}}{R_{3}}}{\frac{R_{n}}{R_{1}}+\frac{R_{n}}{R_{2}}+\frac{R_{n}}{R_{3}}+\frac{R_{n}}{R_{4}}}$

Where $Q_{n}$ is the flowrate through the $\mathrm{n}$-th inlet channel, $R_{n}$ is the resistance of the n-th inlet channel and $P_{n}$ is the pressure applied to the n-th inlet.

\section{Central stream placement}

We wish to use our system to deliver solutes to certain locations in the main channel by introducing them in the central focused stream (green in 
Figure 2.1) and changing the width and lateral position of this stream. The resistor model allows us to determine the flow rates in terms of the inlet pressures, and we will now use it to determine the inlet pressures needed at the three inlets for a specific width and lateral position of the central focused stream. To uniquely determine the three controlled pressures we develop three constraints: width, position and velocity of the central stream.

For a plug flow profile in a rectangular channel, the fraction of flow that a given inlet contributes to the total flow equals the fractional width of the stream it produces with respect to the total channel width. So, for our system we can write the width of the $n$-th stream as:

$2.5 \quad W_{n}=\frac{Q_{n}}{Q_{1}+Q_{2}+Q_{3}} W_{\text {total }}$

Where $W_{n}$ is the width of the $\mathrm{n}$-th stream in the main channel and $W_{\text {total }}$ is the total width of the main channel.

The distance between the center of the focused stream and the wall that borders stream $1(x)$ can be written as :

$$
x=\left(\frac{Q_{1}}{Q_{1}+Q_{2}+Q_{3}}+\frac{1}{2} \frac{Q_{2}}{Q_{1}+Q_{2}+Q_{3}}\right) W_{\text {total }}
$$

And finally we constrain the linear velocity $(v)$ in the main channel by applying the condition:

$$
2.7 \quad v=\frac{Q_{1}+Q_{2}+Q_{3}}{A_{\operatorname{main}}}
$$

where $A_{\text {main }}$ is the cross-sectional area of the main channel. Equations 2.5, 2.6 and 2.7 are then solved simultaneously after substituting equation 2.5 for each flow rate to determine the input pressures $\left(P_{1}, P_{2}\right.$, and $\left.P_{3}\right)$ required to generate the specified conditions.

\section{Concentration profile of diffusing compounds}

We are also interested in determining how diffusion affects the concentration profiles we can create with this technique. As the streams progress through the channel, the solutes they carry will begin to diffuse causing their concentration profile to spread, thus limiting our ability to keep the concentration regions local. To calculate the $2 \mathrm{D}$ (where $\mathrm{x}$ is taken as the lateral distance and $y$ as the downstream distance) concentration profile in the channel, we will consider it as a series of 1D concentration profiles translating 
down the channel at the flow velocity. If the flow rate changes, the concentration profiles require a different amount of time to move a fixed distance downstream. As diffusion has had more or less time to act, the spread of the profile at that point thus is changed when the flow rate changes.

If we assume that the diffusivity of the solute does not depend on the flow rate, the textbook[39] definition of the time evolution of a 1D profile that begins as a perfect plug is:

$2.8 \quad c_{t}\left(x_{c}, t\right)=\frac{c_{o}}{2}\left(\operatorname{erf}\left(\frac{x_{c}+\Delta x_{c}}{\sqrt{4 D t}}\right)-\operatorname{erf}\left(\frac{x_{c}-\Delta x_{c}}{\sqrt{4 D t}}\right)\right)$

Where $x_{c}$ is the distance from the center of the middle stream $\Delta x_{c}$ is the width of the plug, $D$ is the diffusion coefficient of the diffusing species, $t$ is the time since the plug was introduced into the system and $c_{o}$ is the initial concentration of the solute considered.

If we consider the profile to be translating at a constant velocity we can rewrite equation 2.8 as:

$$
c_{t}\left(x_{c}, y\right)=\frac{c_{o}}{2}\left(\operatorname{erf}\left(\frac{x_{c}+\Delta x_{c}}{\sqrt{\frac{4 D}{v} y}}\right)-\operatorname{erf}\left(\frac{x_{c}-\Delta x_{c}}{\sqrt{\frac{4 D}{v} y}}\right)\right)
$$

Where $v$ is the linear flow velocity in the channel and $y$ is the distance downstream from the junction.

We will use this equation to predict the concentration profile at a given distance downstream from the junction. Equation 2.9 implies that flow velocity, diffusivity and distance from the junction are the key factors for controlling the spread of the starting profile in this technique.

\section{Materials and Methods}

\section{PDMS Device Fabrication}

Polydimethylsiloxane (PDMS) devices were fabricated with soft lithography. Briefly, chip geometry was defined via lithography of 100 micrometers of SU8 on a silicon wafer. Inlet channels were $500 \mu \mathrm{m}$ wide while the channel after the junction was $1.5 \mathrm{~mm}$ wide. PDMS (Sylgard 184) with a 10:1 elastomer to curing agent ratio was poured over the mold and left to set for 3 hours at $60^{\circ} \mathrm{C}$. The PDMS was then removed from the mold, and $1 \mathrm{~mm}$ inlets were made with a biopsy punch (Harris Uni-Core). The chips were then 
activated in an oxygen plasma cleaner (Harrik Plasma) for 45 seconds at high power (30 Watts) and bonded to similarly treated glass microscope slides (Corning).

For better adhesion between the PDMS and collagen, the surface of the chips were silanized with (3-aminopropyl)triethoxysilane (APTES) and treated with glutaraldehyde. To do this, the chips were first submerged in a $10 \% \mathrm{w} / \mathrm{w}$ APTES (Sigma-Aldrich) in water solution for $30 \mathrm{~min}$. Devices were then briefly rinsed with $90 \%$ ethanol before being submerged in $10 \% \mathrm{w} / \mathrm{w}$ glutaraldehyde (Sigma-Aldrich) in phosphate-buffered saline (PBS, SigmaAldrich) for another $30 \mathrm{~min}$. Finally, the chips were filled with a $4 \mathrm{mg} / \mathrm{mL}$ solution of titrated rat tail collagen I (Corning) and cured for 2 hours at $36^{\circ} \mathrm{C}$.

\section{D Printed Chip Fabrication}

The 3D version of the device was printed in Clear Resin (FormLabs) on a Formlabs Form 2 3D printer. Channel dimensions were $1 \times 1 \mathrm{~mm}$ for the inlets and $2 \times 2 \mathrm{~mm}$ for the channel after the junction. The surface was treated similarly to the PDMS chip for proper gel adhesion. The devices were first treated with oxygen plasma to clean the surface. Then they were submerged in a acrylamide/bis-acrylamide solution to add primary amines to the surface. The composition of coating solution used here was $9 \mathrm{ml} 40 \%$ Bis-acrylamide (BioRad), $30 \mathrm{ml}$ of PBS and $22.5 \mu \mathrm{L}$ of $10 \%$ ammonium persulfate (APS, BioRad) The chips remained submerged for $30 \mathrm{~min}$. Then they were rinsed with $90 \%$ ethanol, treated with the glutaraldehyde solution mentioned above and filled with the titrated collagen solution mentioned above. After curing for 2 hours at $60^{\circ} \mathrm{C}$ the devices were ready to use.

\section{Experimental setup}

All devices shown here consist of a series of inlet channels ( 3 inlets for the $2 \mathrm{D}$ chips and 5 inlets for the 3D chips) that combine at a junction to become the single wider main channel of the device. Chip inlets were fitted with $200 \mu \mathrm{l}$ pipette tips as reservoirs. Pressure was applied to the reservoirs via Tygon pneumatic tubing by a Fluigent pump (MFCS-EX). For the positional and width control tests in 3D and 2D chips, side inlet reservoirs were filled with PBS while the center inlet reservoir contained $0.1 \mathrm{mg} / \mathrm{ml}$ of $40 \mathrm{kDa}$ fluorescein isothiocyanate (FITC)-labeled dextran (Sigma-Aldrich) in PBS. For the time response experiment, the side channel reservoirs still contained PBS while the center channel reservoir contained a cocktail of $0.1 \mathrm{mg} / \mathrm{ml}$ Alexa Fluor 647 
carboxylic acid, tris(triethylammonium) (Sigma-Aldrich) and $0.1 \mathrm{mg} / \mathrm{ml}$ of 40 kDa FITC-labeled dextran in PBS.

\section{Experimental Parameters}

Positional control, width control and time response experiments were run in the $2 \mathrm{D}$ devices. Before a chip was used for an experimental run, a single 2 min calibration was done to determine the resistances of the three inlet channels and of the main channel of the chip. Details of the calibration can be found in Appendix 1. From these calibrations we can also estimate a gel Darcy permeability of $4.7 \times 10^{-10} \mathrm{~cm}^{2}$ which lies within the range of those found in the literature for similar gels. ${ }^{9}$ After calibration, equations $2.5,2.6$, and 2.7 were used to calculate the pressures required to set: the position of the center stream, the width of the center stream, and the flow velocity. The pressures used to generate the shown concentration profiles fell in the range of 0-25 $\mathrm{mBar}$ depending on the device used and the desired profile. Width control was validated by setting the central stream width to $5 \%, 10 \%, 20 \%, 30 \%$ and $40 \%$ of the total channel width, with the stream centered in the channel and a flow velocity of $10 \mu \mathrm{m} / \mathrm{s}$. Similarly, to test positional control, the stream width was fixed at $20 \%$ of the channel width, the flow velocity was fixed at $10 \mu \mathrm{m} / \mathrm{s}$ and the distance of the center of the stream to the left wall was adjusted from 150 $\mu \mathrm{m}$ to $1,350 \mu \mathrm{m}$ in increments of $300 \mu \mathrm{m}$. For each tested condition, the flow was allowed to fully develop for 10 min before the measurement was taken and a new condition was chosen. For both experiments the resulting fluorescence profile was recorded and compared to the desired "target" profile.

The target concentration profile requires some adjustment to account for diffusion in the case of the width control experiments. To do this we first measured the diffusion coefficient of the FITC dextran, as described in Appendix 1, and obtained a value of $5.5 \cdot 10^{-11} \mathrm{~m}^{2} \mathrm{sec}^{-1}$, a similar value to those found in the literature.[24] This value was used with equation 2.9 to determine the expected width of the concentration profile at $50 \%$ of maximum concentration at the measurement location. The measurement location was chosen to be $1500 \mu \mathrm{m}$ downstream from the junction as at this point the flow had been fully developed for $\sim 1000 \mu \mathrm{m}$. We considered this the target profile width reported in Figure 2.3.

In the case of the time response experiments the width and flow rate were fixed at the same values as the positional control experiment, but the 
target distance was instantaneously alternated from $150 \mu \mathrm{m}$ to $1,350 \mu \mathrm{m}$ every 45 seconds. Finally, the 3D chips were run uncalibrated; the pressures needed to locate the stream in the upper right corner, the center and the lower left corner of the main channel were estimated from the $2 \mathrm{D}$ experiments.

All quantitative measurements of the profile were taken at $1500 \mu \mathrm{m}$ downstream from the junction and all correlation coefficients between measured data and predicted values $\left(R^{2}\right)$ were calculated with MATLAB.

\section{Results and Discussion}

We explored the effectiveness of our technique for controlling the position and width of co-flowing streams in a gel-filled chip. Effectiveness was evaluated by measuring the accuracy with which we could produce a desired concentration profile in the main channel of a gel-filled three-inlet device by manipulating only the inlet pressures. For a given concentration profile the model described in the theoretical section was used to determine the appropriate inlet pressures. These pressures were then applied to a device with the fluorescent dextran solution loaded in the central inlet channel and the resulting profile was compared to the desired profile.

In addition, we report a brief characterization of the time response of our system and an extension of our 2D device to a fully 3D device.

\section{Positional Control of Concentration Profile}

To demonstrate control of the lateral position of the central stream, we tested 5 target profiles as shown in Figure 2.2. For each profile the width of the concentrated region and the total flow velocity were kept constant and only the lateral position was adjusted. We then measured the center position of the concentration profile and compared it to the target center position (Figure 2.2, top).

The direct agreement between target and measured position was strong $\left(R^{2}=0.97\right)$ with an average difference between measured and target position of 50 microns. It is worth noting that in the case of the far left and far right profiles the measured position shows a marked deviation from the target towards the center of channel. To explain this, we consider the effect of diffusion on the measurement. If the concentrated region is far from the walls of the chip, as it is for the middle profiles, diffusion spreads the profile symmetrically and the center position is unaffected. In the case of the far left 


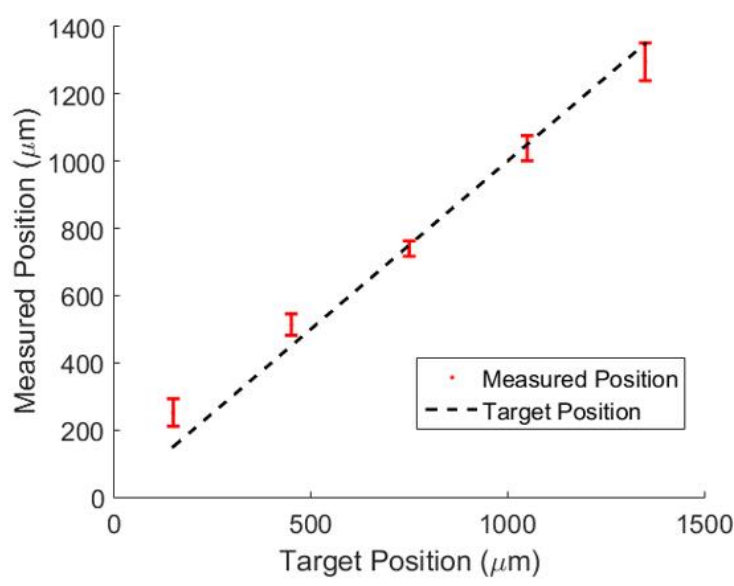

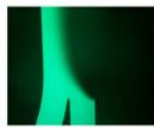

Far Left

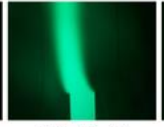

Mid Left

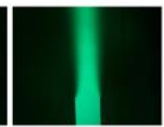

Middle

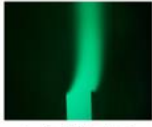

Mid Right

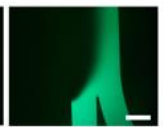

Far Right

Figure 2.2: Positional control of laminar streams inside a hydrogel-filled microfluidic channel. Top, measured center position of the dye-filled stream for 5 different positions plotted against the target position. Error bars are one standard deviation, for 3 measurements each taken on a separate chip. Dotted line represents perfect agreement between target and measured position. Bottom, sample fluorescence intensity profiles from one of the chips. Note that at the far right and far left the left and right inlet channel respectively have stagnated allowing the dye to diffuse upstream, into the neighboring inlet. Scale bar is $500 \mu \mathrm{m}$.

and far right profiles, the concentrated region is flush with the wall of the channel, so the profile can only diffuse in the direction of the center of the channel. This asymmetric spreading shifts the center of the profile slightly towards the center of the channel as is reflected in the data.

\section{Width Control of Concentration Profile}

To demonstrate control of the width of the focused stream, we tested 5 target concentration profiles shown in Figure 2.3 (bottom). For each profile, the position of the concentrated region and the flow velocity were kept constant while only the width of the concentrated region was varied. We then plotted the measured width of the profile at $50 \%$ of maximal intensity against 
the target width of the profile (Figure 2.3, top). The inlet pressures applied to generate the target width were derived from equation 2.4 and adjusted to correct for diffusion as predicted by equation 2.9. The method used for correction is outlined in the methods section.

We found a much weaker agreement between target and measurement $\left(R^{2}\right.$ of 0.78$)$ than that seen for positional control, with the measured width consistently larger than the target width. Average difference between measured and target width was 62 microns. We expect that the observed difference between target and measured width can be explained by a combination of hydrodynamic effects at the junction and the diffusion in the main channel. Despite this inaccuracy, control of the width is relatively precise. This can be concluded from Figure 2.3 where the deviation in measured width
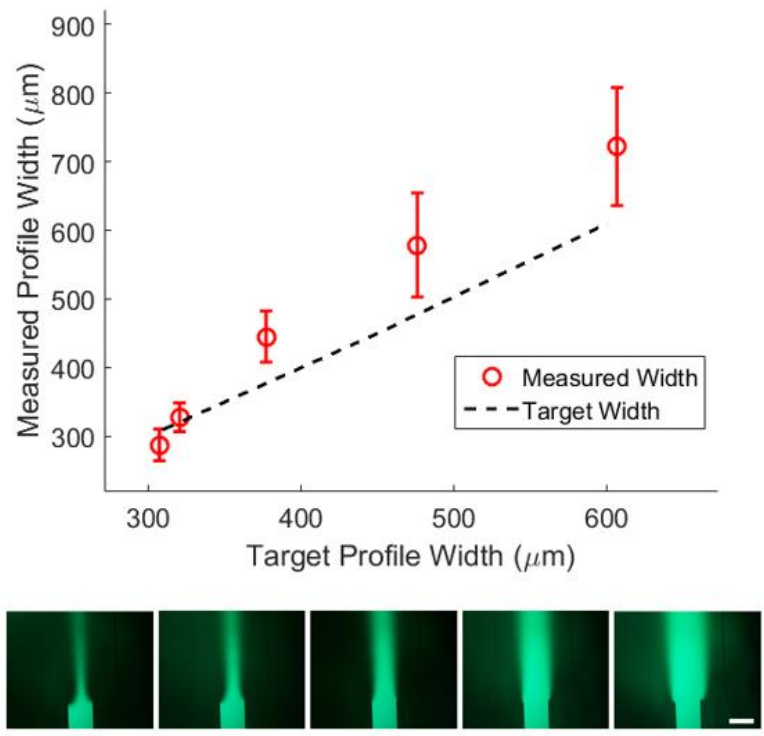

$300 \mu \mathrm{m}$

$320 \mu \mathrm{m}$

$370 \mu \mathrm{m}$

$470 \mu \mathrm{m}$

$600 \mu \mathrm{m}$

Figure 2.3: Width control of laminar streams inside a hydrogel-filled microfluidic channel. Top, width at 50\% maximal intensity, measured $1.5 \mathrm{~mm}$ from the junction for 5 different target widths plotted against the target width for the center stream. Error bars are one standard deviation, for 3 measurements each taken on a separate chip. Linear fit is plotted over the data. Bottom, sample fluorescence intensity profiles from a single chip for a range of target stream widths from $5 \%$ of the channel width up to $40 \%$ of the channel width with the target profile widths listed below. Scale bar is $500 \mu \mathrm{m}$. 
from device to device (error bars) is small compared to the deviation from the target width. Furthermore, measured width and target width are strongly correlated. These combined imply that the inaccuracy could be modeled and calibrated for in future work.

We include a more detailed theoretical discussion of factors affecting profile broadening in Appendix 1. Summarizing this information: faster flow, a shorter main channel, or a more sharply resolved device geometry near the junction leads to less profile broadening and a steeper concentration gradient perpendicular to the flow direction. These factors can be of critical importance when adapting this technique to patterning techniques which require fine resolution with small, more diffusive molecules.

\section{Time Response of Concentration Profile Position}

When the inlet pressures of the system are changed, the change in flow profile downstream is not instantaneous. To characterize the time response we ran a series of tests where a reciprocating pattern was generated in the main channel of the device (Figure 2.4). In these tests we alternated between two target profiles while keeping the flow rate and width of the concentrated region constant. Each state was held for 45 seconds before switching back to the previous state over the course of $<1$ second. We assumed that the delayed response of the system comes from a combination of the relaxation time of the gel,[28] the compliance of the Tygon tubing used and the flow resistance of the gel-filled device. To characterize the net effect, an RC time of $61.8 \mathrm{sec}$ was calculated.

This response time did not significantly affect our ability to generate the standing concentration profiles shown in Figures 2.2 and 3. A response time of one minute is negligibly short compared to the $\sim 2$ days (55 hours) of time needed to drain the $0.1 \mathrm{ml}$ reservoirs of our device at the typical flow velocity of $10 \mu \mathrm{m} / \mathrm{s}$. For cell cultures it is furthermore not expected that time constants of less than a minute are needed. If needed, a number of measures can be taken to improve the response time of the devices. The gel-filled inlet channels were longer than necessary to facilitate interfacing and the pneumatic tubing used was flexible Tygon. For applications where a faster response time is necessary, the inlet resistance could be decreased by using shorter inlet channels and the compliance of the Tygon tubing used could be reduced by using shorter and stiffer tubing. 


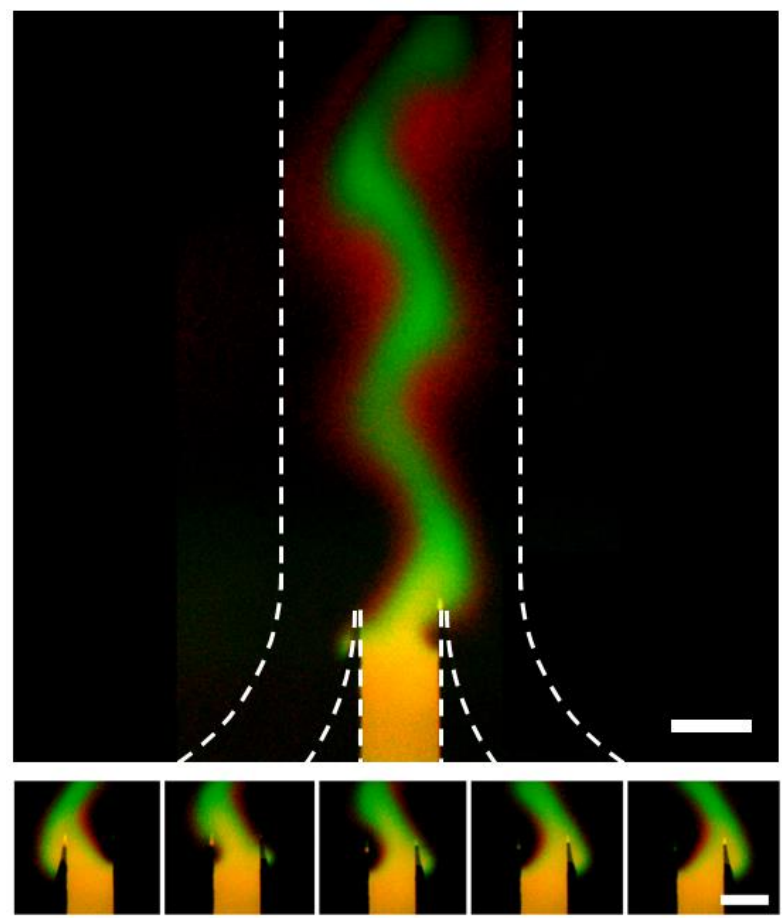

Figure 2.4: Dynamic control over stream position inside a hydrogel-filled microfluidic channel. Dotted lines indicate the walls of the channel. Target profile was switched every 45 seconds and flow rate was $20 \mu \mathrm{m}$ per second. A red (Alexa Fluor 647) and green (FITC-labeled $40 \mathrm{kDa}$ dextran) dye were included in the central inlet for this test. The green channel has been subtracted from the red channel and contrast enhanced to highlight the fact that the $1 \mathrm{kDa}$ red dye diffuses much faster than the $40 \mathrm{kDa}$ green dye. Top, picture of both the junction and the main channel. Bottom, 5 images of the junction taken at 8.7 second intervals showing the stream shift from far left to far right orientation. Raw data is shown in figure A1.5. Scale bar is 500 $\mu \mathrm{m}$.

It is worth noting here that a simple, long-term stability experiment was also run where a static position was chosen and a $10 \mu \mathrm{m} / \mathrm{s}$ flow was maintained for 5 hours. In this test, the variation in position was $2 \%$ of the initial values. See figure A1.3 in appendix 1 for further information. 

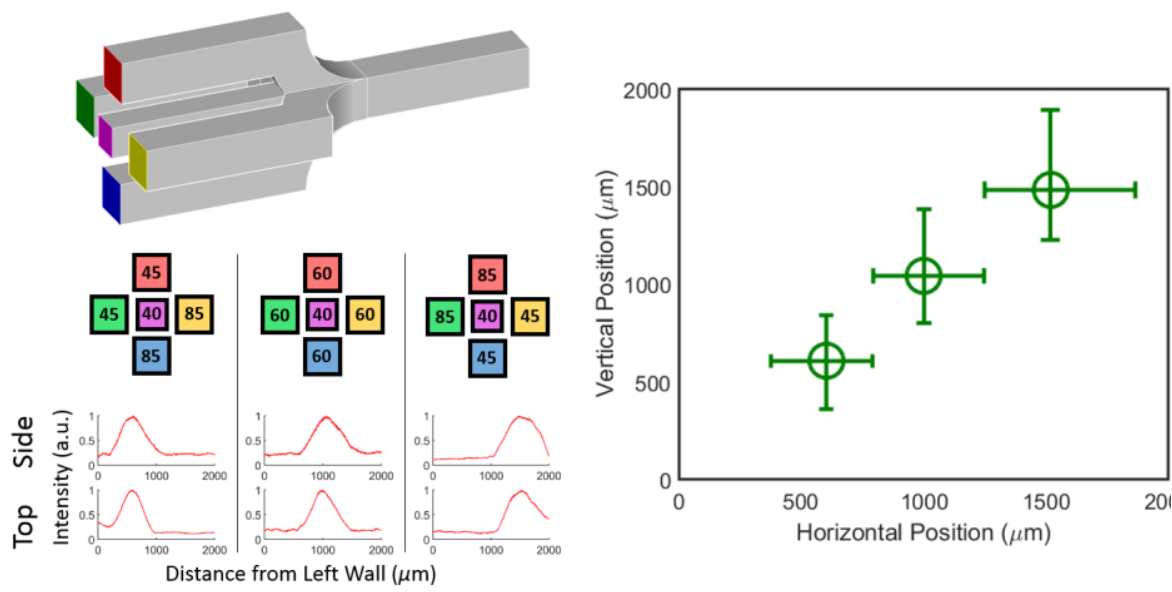

Figure 2.5: Top left, Rendering of the 3D junction with inlets color coded. Middle left, pressures in mBar used to generate the fluorescence profiles shown in the bottom Left. Bottom left, actual fluorescence intensity profiles taken from the bottom and side of the devices during experimental runs. Right, 2D position of the stream at a cross section for 3 different test conditions. Each point represents a vertical position and horizontal position measurement for a single inlet pressure configuration shown on the left. Bounds of the graph are the bounds of the main channel. Error bars are one standard deviation of the respective fluorescent profile.

\section{Extension to 3D: Controlling Stream Position in Both Horizontal and Vertical Direction}

While a 2D junction can be used to manipulate a concentration profile in one dimension, a 3D junction can be used to manipulate a concentration profile in two dimensions. For example, consider a junction where two extra inlet channels are included that enter at the top and bottom of the center inlet channel as shown schematically in Figure 2.5 top left. The central stream can still be localized horizontally as before, but with these added channels the central stream can be localized vertically as well.

To show that a 3D chip with five inlets is truly capable of generating controllable concentration profiles we generated three distinct profiles and imaged them from both the bottom and side of the chip. The position and width of the resulting profiles are represented in Figure 2.5 (bottom). The three distinct horizontal positions ( $x$ axis) show that the position can in fact be controlled by the pressure applied to the left and right inlets just as with the 
2D device. The simultaneously plotted vertical positions (y axis) are distinct from each other and independent of the horizontal position showing that the chip allows full 3D control of the profile. The raw data and pressures used can be found in Appendix 1.

\section{Conclusion}

Previously, laminar flow patterning has been used to locally deliver chemicals to flat surfaces in open microfluidic devices.[1] Here we have shown that $2 \mathrm{D}$ and $3 \mathrm{D}$ laminar flow patterning through a cured hydrogel is not only possible, but relatively simple and precise to control due to the plug flow profile. Through testing of our devices we demonstrate excellent spatial control of the generated concentration profile via manipulation of the inlet pressures and are able to characterize the time response of the system. Furthermore, we show that the technique can be used in a truly 3D capacity by 3D printing and testing a version of our device with five inlets for both horizontal and vertical control of the concentration profile.

Although we have only shown the ability to manipulate concentration profiles in a gel, we hope to see this technique developed as a general way to influence $3 \mathrm{D}$ cell cultures in a gel or $2 \mathrm{D}$ cell cultures on a gel/media interface. Our chips could see immediate use as a platform for locally dosing cells in 3D culture with morphogens to observe the effects of the concentration profile independently from the effects of the interstitial flowrate. Furthermore, we can generate standing gradients of morphogens perpendicular to the direction of interstitial flow, a relatively unprobed combination of stimuli. The ability to very locally dose cell cultures may also provide new techniques for guiding the development of tissues in organs-on-chips. $[29,30,31]$

In addition to biological applications, our technique provides a general microfluidic engineering tool to translate surface patterning techniques compatible with laminar flow patterning to a 3D matrix. For example, instead of patterning channels in 2D with soft lithography, our method would allow channels to be locally etched in a desired geometry by controlling the concentration profile of an etchant like collagenase. Also, instead of relying on $2 \mathrm{D}$ top down patterning techniques, our method would allow control over local stiffness of gels from the inside out by manipulating the concentration profile of a cross-linker within the gel. 
In summary, our technique is a powerful extension of traditional laminar flow patterning from patterning of $2 \mathrm{D}$ surfaces to patterning of $3 \mathrm{D}$ gels. We anticipate that it will find many applications both as a general technique for patterning biological hydrogels and in particular for probing the effects of interstitial flow and morphogen gradients in 3D cell culture models and organs-on-chips.

\section{Acknowledgments}

We would like to acknowledge the European Research Council (ERC) under the Advanced Grant 'VESCEL' Program (Grant no. 669768) of prof. Van den Berg for supporting this work, as well as Vasilis Papadimitriou, Mathieu Odijk, and Jorien Berendsen for thorough discussions and support during this work. 


\section{References}

${ }^{1}$ P. J. A. Kenis, R. F. Ismagilov, and G. M. Whitesides, Science, 1999, 285, 83.

2 Takayama, J. C. McDonald, E. Ostuni, M. N. Liang, P. J. A. Kenis, R. F. Ismagilov and G. M. Whitesides, Proceedings of the National Academy of Sciences, 1999, 96, 5545.

3 D. J. Beebe, J. Moore, J. M. Bauer, Q. Yu, R. H. Liu, C. Devadoss and B. H. Jo, Nature, 2000, 404, 588.

${ }^{4}$ M. Shen, H. Yang, V. Sivagnanam and M. A. M. Gijs, Analytical Chemistry, 2010 82, 9989.

5 S. Ramanujan, A. Pluen, T. D. McKee, E. B. Brown, Y. Boucher and R. K. Jain, Biophysical Journal, 2002, 83, 1650.

6 J. R. Levick, Quarterly Journal of Experimental Physiology, 1987, 72, 409.

${ }^{7}$ D. T. Eddington and D. J. Beebe, Advanced Drug Delivery Reviews, 2004, 56, 199.

8 B. Ziaie, A. Baldi, M. Lei, Y. Gu and R. A. Siegel, Advanced Drug Delivery Reviews, 2004, 56, 145.

${ }^{9}$ M. A. Swartz and M. E. Fleury, Annual Review of Biomedical Engineering, 2007, 9, 229.

$10 \mathrm{~J}$. M. Rutkowski and M. A. Swartz, Trends in Cell Biology, 2007, 17, 44.

${ }^{11}$ M. A. Swartz, Advanced Drug Delivery Reviews, 2001, 50, 3.

12 J. H. E. Cartwright, O. Piro and I. Tuval, Proceedings of the National Academy of Sciences, 2003, 101,7234

13 J. M. Munson and A. C. Shieh, Cancer Management and Research, 2014, 6, 317.

${ }^{14}$ W. J. Polacheck, J. L. Charest and R. D. Kamm, Proceedings of the National Academy of Sciences, 2011, 108, 11115.

${ }^{15}$ C. P. Ng and M. A. Swartz, American Journal of Physiology: Heart and Circulatory Physiology, 2003, 284, 1771.

${ }^{16}$ A. M. Kloxin, A. M. Kasko, C. N. Salinas and K. S. Anseth, Science, 2009 324, 59.

${ }^{17}$ B. M. Baker and C. S. Chen, Journal of Cell Science, 2012, 125, 3015.

18 S. Trietsch, G. D. Israëls, J. Joore, T. Hankemeier and P. Vulto, Lab on a chip, 2013, 13, 3548.

${ }^{19}$ B. Gumuscu, J. G. Bomer, A. van den Berg and J. C. T. Eijkel, Lab on a Chip, 2015, 15, 664.

${ }^{20}$ S. Chung, R. Sudo, V. Vickerman, I. K. Zervantonakis and R. D. Kamm, Annals of Biomedical Engineering, 2010 38, 1164.

${ }^{21}$ E. Palleau, D. Morales, M. D. Dickey and O. D. Velev, Nature Communications, 2013, $4,1$.

${ }^{22}$ C. C. Lin, \& K. S. Anseth, Pharmaceutical Research, 2009, 26, 631.

${ }^{23}$ V. S. Shirure, A. Lezia, A Tao, LAlonzo and S.C. George, Angiogenisis, 2017, 20, 493.

${ }^{24}$ R. Sudo, S. Chung, I.K. Zervontonakis, V. Vikerman, Y. Toshimitsu, L. G. Griffith, R. D. Kamm, FASEB Journal, 2009, 23, 2155.

${ }^{25}$ E. M. Johnson, D. A. Berk, R. K. Jain and W. M. Deen, Biophysical Journal, 1996, 70, 1017. 
${ }^{26}$ R. F. Ismagilov, A. D. Stroock, P. J. A. Kenis, G. Whitesides and H. A. Stone, Applied Physics Letters, 2000, 76, 2376.

${ }^{27}$ A. Kamholz, B. Weigl and B. Finlayson, Analytical Chemistry, 1999, 71, 5340.

${ }^{28}$ D. M. Knapp, V. H. Barocas, A. G. Moon, K. Yoo, L. R. Petzold and R. T. Tranquillo, 1997, Journal of Rheology, 41, 971.

${ }^{29}$ A. D. van der Meer and A. van den. Berg, Integrative Biology, 2012, 4, 461

${ }^{30}$ S. N. Bhatia and D. E. Ingber, Nature Biotechnology, 2014, 32, 760.

${ }^{31}$ D. Huh, G. A. Hamilton and D. E. Ingber, Trends in Cell Biology, 2011, 21, 745.

${ }^{32}$ K. C. Boardman and M. A. Swartz, Circulation Research, 2003, 92, 801.

${ }^{33}$ A. C. E. Helm, M. E. Fleury, A. H. Zisch, F. Boschetti, M. A. Swartz and R. Langer, Proceedings of the National Academy of the Sciences, 2017, 102, 15779

34 J. D. Shields, M. E. Fleury, C. Yong, A. A. Tomeri, G. J. Randolph and M. A. Swartz, Cancer Cell, 2007, 11, 526-538

${ }^{35}$ R. J. Phillips, W. M. Deen, and J. F. Brady, Journal of Colloid and Interfacial Science, 1990, 139, 363-373

${ }^{36}$ L. Durlofsky, and J. F. Brady, Physics of Fluids, 30, 3329-3341

37 J.R. Levick, Quarterly Journal of Experimental Physiology, 1987, 72, 409-438

${ }^{38}$ V. van Duinen, S. J. Trietsch, J. Joore, P. Vulto, T. Hankemeier, Current Oppinion in Biotechnology, 2015, 35, 118-126

${ }^{39}$ Crank, J. The mathematics of diffusion. Oxford: Clarendon Press, 1976

${ }^{40}$ S. N. Bhatia, D. E. Ingber Nature Biotechnology, 2014, 32, 760-772

${ }^{41}$ A. van der Meer, A. van den Berg, Integrative Biology, 2012, 5, 461-470

${ }^{42}$ D. Huh, G. A. Hamilton, D. Ingber, Trends in Cell Biology, 2011, 21, 745 


\title{
Chapter 3: Towards Precise Control of Microvascular Geometry in Self- Organized Microvascular Networks
}

\author{
Contributions by: Joshua Loessberg-Zahl, Roger D. Kamm, \\ Albert van den Berg, Andries D. van der Meer, \\ Jan C.T. Eijkel
}

\begin{abstract}
We demonstrate the use of flow patterning to generate controlled concentration profiles in the interstitial space around microvascular networks on chips. Our goal is to show that concentration profiles of growth factors generated in this way are capable of directly modifying the structure of otherwise disordered microvascular networks. This technique should serve as a powerful tool in tissue engineering and a way to precisely study the combined effects of flow and chemical cues on microvascular development. The initial experiments presented here serve as a proof of concept for these future goals. Explicitly we show that we are capable of generating simple gradients over microvascular networks and that generated profiles change little over the length of the vascularized region in our device. To demonstrate the full potential of the technique, our future plans are to fabricate devices capable of generating guided streams of growth factors and use them for precise modification of microvascular networks.
\end{abstract}




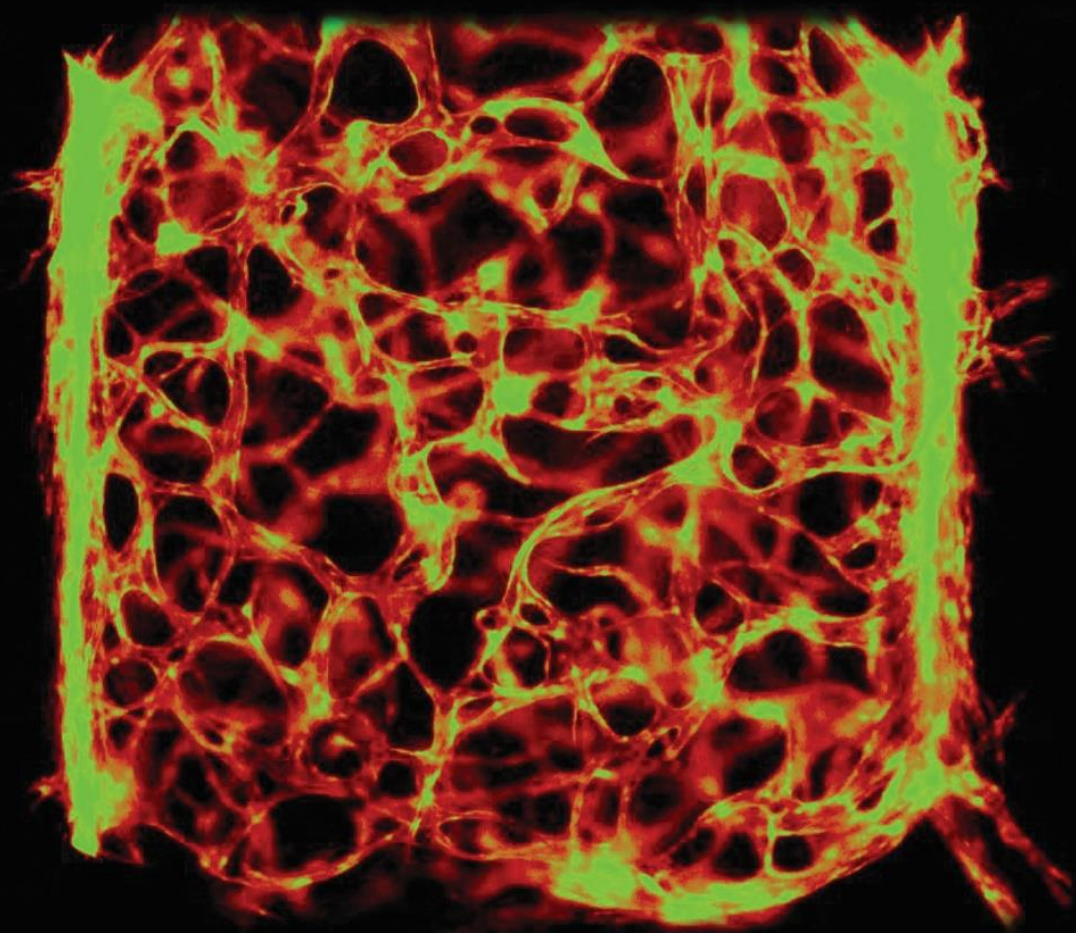




\section{Introduction}

Developers of organ-on-chip technologies are attempting closer and closer mimicry of in vivo systems. The hope is that this progress will improve the use of organ-on-chip technologies for precision medicine and drug development.[1] With this push towards more physiological tissues-on-chips comes a need to make ever more complex vasculature. While the primary function of most vasculature is simply to supply nutrients to tissues and remove waste, there is an increasing recognition that the fine scale geometry of vasculature is important for proper mimicry of function.[2,3] Optimal function of some critical tissues like those of the liver require arrangement of vasculature and surrounding cells on the scale of single cells.[4] Similarly, understanding fine-scale local changes to existing vasculature is critical to understanding the progression of diseases like cancer.[5]

Current techniques for precisely patterning vascular geometry on chip cannot produce microvessels at the same fine scale as found in the body. 3D bioprinting shows promise for creating precise vascular networks, however techniques that allow direct printing of live cells currently have a minimum resolution of $\sim 100$ microns at best. [6] Other techniques for precise control of geometry rely on generating a tunnel through some biocompatible medium and subsequently seeding it with cells. $[2,3,7]$ These techniques suffer from two drawbacks when pushed to small length scales. First, the concentration of cells needed for seeding increases with the surface area to volume ratio of the vascular network and maintaining a homogenous suspension at high concentration becomes difficult. Secondly, small micro vessels often have a diameter that is the same size or smaller than the diameter of an endothelial cell in suspension ( 10 micrometers)[3] which makes it difficult to introduce the cells uniformly or even at all.

As an alternative to these techniques which produce vessels of precise geometry but with relatively large diameters, bottom-up techniques can be used to produce disordered networks of vessels on chip with diameters similar to those of physiological microvasculature.[2,8,9] Some stimuli, both mechanical and chemical, have shown promise for shaping these disorganized networks after their generation [10] but, to our knowledge, these have only been applied broadly to modify entire networks. 
We have developed a platform to apply signaling chemicals to specific regions of an on-chip microvascular network with the goal of locally modifying its morphology. We use our previously described flow-based technique for producing complex concentration profiles inside biological hydrogels [11] and show that it is applicable to the generation of concentration profiles in the interstitium around microvascular networks. While we have yet to achieve the goal of causing morphological changes to a network, we provide the basic proof of concept for the technique, showing that the profiles develop as expected and that the networks do not disturb them. Furthermore we show that the flow is not able to significantly penetrate the endothelium, implying that the luminal and interstitial spaces are independently chemically addressable in this technique. Future work will aim to show that this technique is useful for generating morphological changes in the microvascular networks.

\section{Results and Discussion}

\section{Device Layout and Cell Seeding}

The device used here is designed to allow diffusive nutrient delivery to a vascular network while it develops but still allow active generation of gradients in the interstitium around the network once it is fully developed. Our design is based loosely on designs from the group of R. Kamm.[9] The general layout of the device is shown in Figure 3.1. Adjacent regions of the devices are separated by phase guides $[12,13]$ to keep injected liquids in their intended region.

Each region of the device plays a critical role in the device function. The central region will eventually hold the microvascular network to be manipulated. The gel inlet region is attached to two large reservoirs (not shown in figure 3.1) and allows two flowing streams with different chemical makeup to meet and form gradients. The outlet resistor provides flow resistance and has been tuned such that a $1 \mathrm{~cm}$ liquid height in the gel inlet reservoirs produces a flow velocity $\sim 1 \mu \mathrm{m} / \mathrm{s}$. The gel outlet channel allows this flow to leave the system. The side media channels allow the developing vascular network to receive nutrients during early days of culture, before flow through the gel is applied.

The device is seeded in a particular order to reduce stress on the seeded cells. During day zero, gel regions are filled with $3 \mathrm{mg} / \mathrm{mL}$ fibrin gels. (Figure 3.1, Day 0) The gel regions upstream and downstream of the central 


\section{Layout}

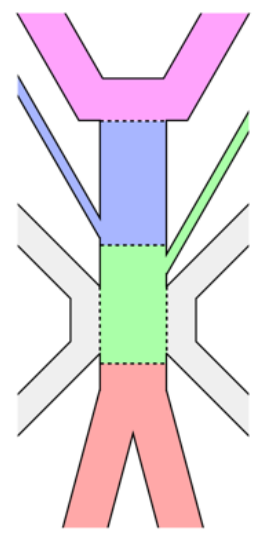

-. Phase Guide

Outlet Resistor

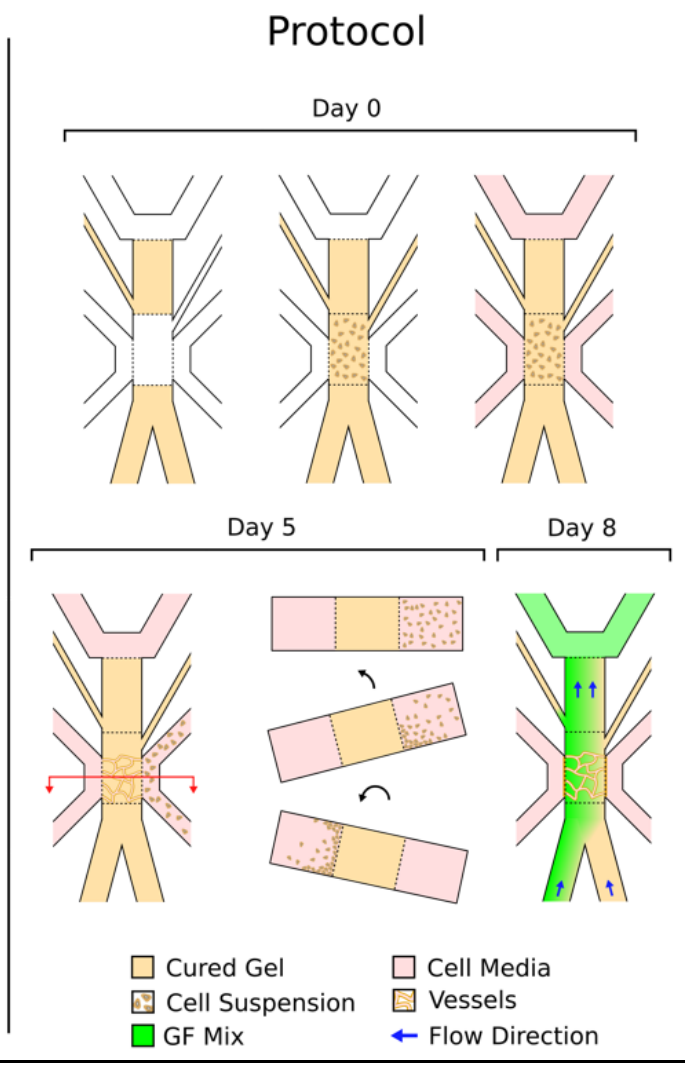

Figure 3.1: Left panel shows the overall layout of the device with various regions labeled. Right panel shows the cell seeding protocol and flow experiments. All gels are injected into their respective regions and media channels are filled on day zero in the order shown. The gels in the upper two regions are injected via narrow gel injection channels. On day 5, monolayers are seeded on the gel-media interface of both media channels. Protocol for ensuring total coverage of the interface is shown in the cross sections on the right side of the day 5 panel. These cross sections are taken at the red line shown in the left side of the day 5 panel. Finally on day 8 , gel inlets are filled, inducing flow through the device and allowing generation of gradients in the gel inlet region.

region are filled and cured first. Gel containing a suspension of 8 million cells per $\mathrm{mL}$ of human umbilical vein endothelial cells (hUVECs) and 1 million cells per $\mathrm{mL}$ of human lung fibroblasts ( $\mathrm{hLFs}$ ) is then introduced to the central region and cured. As a last step, the media channels are filled with cell media. Filling the central region as the last step before adding media prevents any extra 
stress on the cells from dehydration or lack of nutrients. Medium in the media channels is then replaced daily.

After the vascular network has begun to reach the media channels, an endothelial monolayer is seeded on the interface between media channels and the central region (Figure 3.1, Day 5). This monolayer is intended to prevent flow from the central region into the media channels during flow experiments. However, if the monolayer is seeded before the vascular network has opened up to the media channels, it can block transport and starve the growing network. In our devices, the vascular network began to open up to the media channels on day 4 and monolayers were seeded on day 5 . To seed a monolayer, a cell suspension of 1 million hUVECs per $\mathrm{mL}$ was first introduced into one of the media channels. The device was then tilted slightly such that the cells fall into the bottom corner of the gel-media interface as shown in Figure 3.1, Day 5, right. The device was left to sit in this orientation for $10 \mathrm{~min}$ while the cells settle and attach. After this, the device was inverted and tilted again, allowing the cells to settle in the top corner of the gel-media interface. This protocol was then repeated for the second media channel. If this double seeding protocol was not followed, leaks from the central region to the media channels were often observed during flow experiments. The formed layers were observed to be confluent the next day and flow experiments were conducted on day 8.

\section{Flow Experiments}

During flow experiments, interstitial flow was generated by filling the two large reservoirs attached to the gel inlet region to a height of $1 \mathrm{~cm}$. The diameter of the reservoirs was sufficiently large such that the reservoirs only needed to be refilled once a day to keep the resulting 1 micron per second flow within 5 percent of its starting value.

Our first proof-of-concept experiments aimed to show that our technique can reproduce the simple gradients found in 3D cell culture literature.[1] (Figure 3.2) During these experiments one reservoir was spiked with a chemical of interest. Once the flow is fully developed throughout the device, a gradient is formed in the gel inlet region and is transported across the vascularized region by the interstitial flow. (figure 3.2 top) In these proofof-concept experiments we generated gradients of a $10 \mathrm{kDa}$ dextran 
conjugated with a blue fluorescent dye as it has a similar diffusivity to many small signaling proteins used in organ on chip studies. [14]

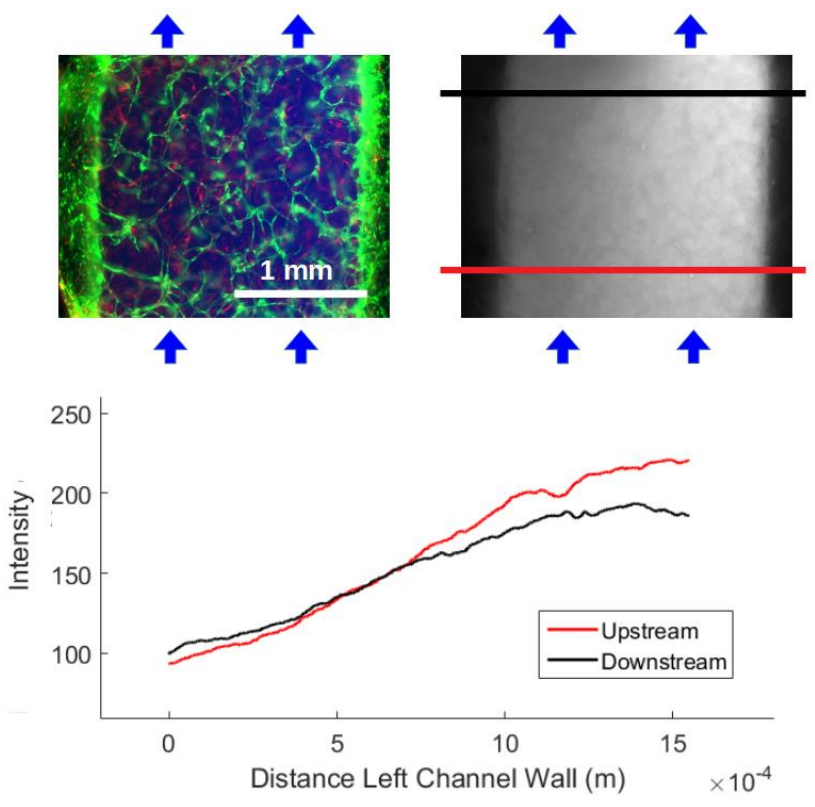

Figure 3.2: Preliminary results showing our ability to generate gradients over microvascular networks in the device shown in figure 3.1. Top left panel shows the vascularized region with fibroblasts expressing RFP, hUVECs expressing GFP and a generated gradient of a blue fluorescent dextran. The signal from the dextran alone is shown in the top right panel. Blue arrows indicate direction of flow. The bottom panel shows intensity profiles taken at the red and black lines in the top right panel. Notice that the profile leaving this region is almost identical to the profile entering. The lack of an extreme shift in the profile indicates that the endothelium is fluidically impermeable relative to the gel and there are no leaks sufficiently large enough to redirect the flow.

In addition to validating our ability to create concentration profiles over microvascular networks, this work validated a key design assumption about the relative permeability of the endothelium. To maintain the desired concentration profile over the entire length of the central region, the flow must not leak significantly into the media containing channels through the endothelial monolayer. If leakage is significant, the concentration profile will either be warped towards any point leaks or will spread out quickly if the endothelium is generally too hydraulically permeable. From literature values 
of permeability,[15] flow through an intact endothelial monolayer and lumens was expected to be about $1 \%$ of flow through the gel. General permeability of the endothelium was therefore not expected to be a problem and we did not observe any effects thereof. However, point leaks in the endothelial monolayers did present a significant challenge. In initial experiments, leaks were observed in the endothelial monolayer and did not reduce in magnitude upon a longer culture time. To combat this leakage, the two-step monolayer seeding protocol mentioned above was introduced. This procedure was aimed to have the suspended cells in the media channels sediment directly into the leak prone corners of the device. This procedure proved successful. Evidence of the resulting leak-free endothelial monolayer can be seen in the bottom panel of figure 3.2. No dramatic broadening or warping towards a leak is observed, implying a relatively impermeable monolayer.

\section{Outlook}

\section{Future work}

The immediate next step of this work will be to show that we can perfuse the vascular networks from the media channels while a gradient is being generated in the interstitium. This would serve as evidence that we can generate the kinds of interstitial gradients commonly used in the literature,[1] while still allowing flow of fluid and even flow of suspended cells through the luminal space. Such a platform would allow the study of phenomena involving circulating cells, like extravasation of circulating tumor cells, but now under gradients of interstitial conditions.

In subsequent steps we would try to test our ability to directly modify the structure of the vasculature using our technique. In the simple gradient generating devices shown here, gradients of chemicals that are known to disrupt normal vascular structures like TNF $\alpha$ or TGF $\beta$ could be applied. These experiments would allow a study of vascular network pruning in which the effects of a wide range of dose and duration could be tested at once. These experiments would also hopefully allow the creation of highly connected networks with graded geometry which are difficult to achieve with current techniques.

As a final test of the technique, we would attempt to make a device with three gel inlets for guiding a single central stream as shown in figure 3.3. Any morphological changes caused by chemicals in this guided stream could 


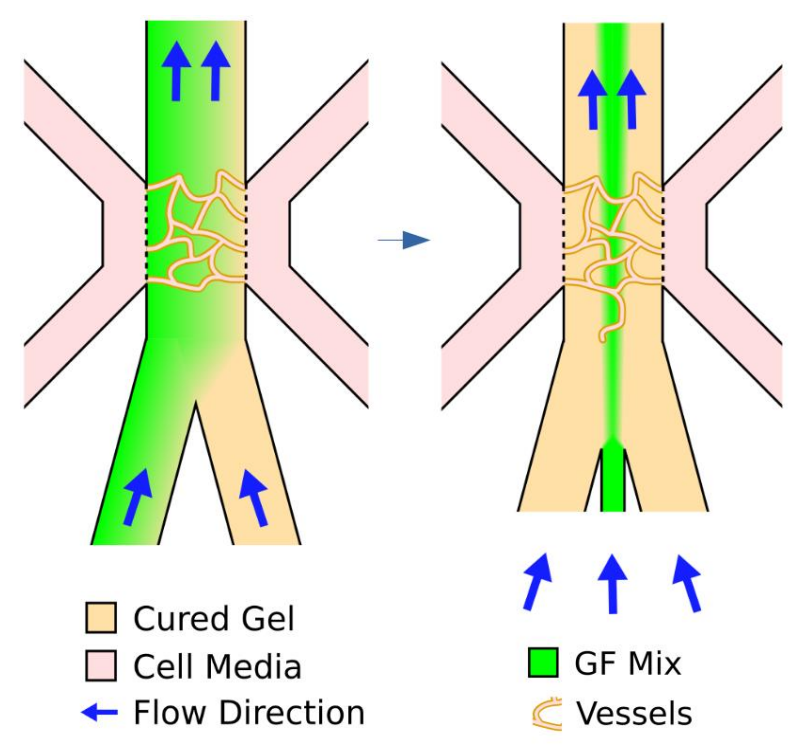

Figure 3.3: Schematic of the current device design and its planned next iteration. The addition of a central gel inlet will allow the focusing and guidance of a central stream of growth factors. We hope to use this focused stream to effect local changes in network morphology and even induce sprouting from the network on a single vessel scale.

then be precisely applied to specific regions of the vascular network. While this may be useful for locally damaging the networks or making networks with hierarchal geometry, we find the potential for control of single vessel growth the most motivating.

Recent work has shown that the combination of flat concentration profiles of growth factors and interstitial flow is sufficient to cause upstream vessel growth from vascular networks $[10,16]$. Therefore, if a growth factorcontaining central stream can be focused to a sufficiently narrow width, it may be possible to induce upstream growth of a single microvessel. This microvessel could then be guided as it grows upstream by adjusting the position of the focused stream. This would allow production of microscale blood vessels with precise and designed geometry.

Using the described three inlet device, we will be able to answer fundamental questions about the growth response of blood vessels to 
simultaneous chemical and mechanical stimuli while working towards controlling the growth of single blood vessels. In particular, comparing the width in which growth occurs to the concentration profile of growth factors will allow us to ascertain the lowest concentration of growth factor needed to induce upstream growth. The specific relation between vessel growth rate and growth factor concentration will tell us if the growth rate is proportional to concentration or if there is simply a concentration threshold, after which growth occurs at a constant rate. The answers to these questions should be interesting as a study of fundamental biology, but will also directly indicate how finely we will be able to focus the region of vessel growth in our system. If further narrowing of this region is required for single vessel growth, angiogenesis inhibitors with known threshold-like behavior[17] can be included in the non growth factor-containing streams.

\section{Conclusion}

We have shown a proof-of-concept design for the manipulation of the concentration profile in the interstitium around microvascular networks. Our technique can recreate concentration profiles commonly used in in the organon-chip literature, but should allow simultaneous perfusion of the microvascular network. In the future, we expect that this technique can facilitate the precise engineering of fine scale microvascular networks and allow the modeling of relatively complex biological phenomena, like the effect on microvasculature of growth factor plumes.

This technique may also find wider application in the fields of organon-chip and tissue engineering. Our design is simple to use and analogous to typical platforms used in 3D cell culture, but uniquely grants us the additional ability to generate complex concentration profiles within the gel. The ability to locally apply growth factors to 3D cell cultures with both spatial and temporal control may be of interest to those working to differentiate stem cells in 3D culture and could help in the design of complex and functional organoids.

\section{Materials and Methods}

\section{Device Fabrication}

Microfluidic devices were made from cast Sylgard 184 PDMS bonded to glass cover slips. The liquid PDMS was mixed at a 10:1 polymer to crosslinker ratio and cast on an acrylic mold. The mold was fabricated by solvent welding thin $\mathbf{5 0 0}$ micron thick pieces of acrylic to a thicker acrylic 
substrate. The thin pieces of acrylic define the channel geometry in the same way that the SU8 does in a conventional PDMS mold. The thin pieces of acrylic were cut with a Trotec 500 laser cutter.

After demolding, all PDMS surfaces were covered with scotch tape to protect them from dust and holes were punched in the PDMS slab to make inlets. The devices and coverslips were then activated in a Harrick oxygen plasma cleaner at high power for 45 seconds and were then bonded by pressing them gently together.

Reservoirs on gel inlets were made from plastic $10 \mathrm{~mL}$ syringes with un-threaded male Luer lock tips. The plungers were removed and syringes were cut down so that the first $1.5 \mathrm{~mL}$ above the tip remain. The tips were then press-fit into the inlets after the devices were filled with hydrogel. If the tips often fell out of the inlets, an extra layer of PDMS with punched holes was added to the bonded device to brace the reservoirs before filling the devices with gel. This slab was $\sim 5 \mathrm{~mm}$ thick and was cut down to be large enough to cover both inlets well but was not larger than the area of the device. This slab was then punched with the same biopsy punch as the inlets at the same spacing of the inlets. Both the bonded device and the slab were then activated in the same plasma cleaner and the slab was bonded to the device such that its holes lined up with the inlet holes in the device.

If any of the bonding steps failed, the substrates needed to be mechanically cleaned before bonding. This was best done by wetting a particulate-free wipe with isopropyl alcohol and then gently rubbing the bonding surface. Before the alcohol could evaporate, the surface was rinsed with ethyl alcohol and before this could evaporate with deionized water. The surface was then blown dry with particulate-free (filtered) compressed air.

\section{Gel Preparation}

Hydrogels used were $3 \mathrm{mg} / \mathrm{mL}$ fibrin gels. To make the gel, a $6 \mathrm{mg} / \mathrm{ml}$ solution of fibrinogen (Sigma Aldrich) in Phosphate Buffered Saline (PBS) (Sigma Aldrich) was prepared by mixing the dry fibrinogen with the PBS and heating the mixture to 36 degrees Celsius for $30 \mathrm{~min}$ until the fibrinogen dissolved. After dissolution the solution was chilled on ice. A $4 \mathrm{U} / \mathrm{mL}$ thrombin (Sigma Aldrich) in Endothelial Growth Media (EGM) (Sigma Aldrich) solution was then also prepared on ice. Finally the solutions were mixed 1:1 and injected into the appropriate gel regions of the waiting device. The device was 
transferred immediately to a humidity chamber [9] and placed in an incubator at 36 degrees Celsius to cure for at least $10 \mathrm{~min}$.

\section{Cell Seeding}

The microvascular network forming protocol used here was based off of 3D culture work by the group of R. Kamm [9], and should be adapted to the relevant experimental conditions. To make the microvascular networks, gels were prepared as stated above, but thrombin/EGM solutions included suspended cells: 16 million/mL GFP expressing human umbilical vein endothelial cells (Lonza) and 2 million/ml RFP expressing human lung fibroblasts (Lonza). These cell concentrations were halved when the cell containing thrombin/EGM solution was mixed 1:1 with the fibrinogen solution. After cell-gel mixtures were injected into the central region of the device, the device was transferred immediately to a humidity chamber and placed in an incubator at 36 degrees Celsius to cure for at least $10 \mathrm{~min}$. The media channels were then filled with EGM. This media was replaced daily.

Monolayers were seeded on day 5 after gel filling. This was done one media channel at a time. A 1 million cell $/ \mathrm{mL}$ suspension of human umbilical vein endothelial cells was injected into the media channel to be seeded and the device was tilted slightly such that the cells would sediment out onto the bottom corner of the media-gel interface. After 10 minutes, the devices were flipped upside down and tilted again such that cells would sediment out onto the top corner of the media-gel interface. The formed monolayers were allowed to mature for a further 3 days before gradient experiments were performed.

\section{Flow Experiments}

Before experiments, reservoirs attached to the gel inlet region were filled. 1 micron per second flow through the gel was generated by filling the reservoirs with EGM up to a height of $1 \mathrm{~cm}$ above the device. Media in one of the reservoirs was mixed with a $10 \mathrm{kDa}$ Cascade Blue labeled dextran (ThermoFisher Scientific). Devices were left in an incubator for three hours to allow flow to fully develop before imaging. The devices were taken to a microscope for imaging. In this case we used a (Olympus FV1000) but any normal epifluorescent microscope equipped with the correct filters and light source should be sufficient. 


\section{References}

${ }^{1}$ V. van Duiden, S. J. Trietsch, J. Joore, P. Vulto, T. Hankemeier, Current Oppinion in Biotechnology, 2015, 35, 118.

${ }^{2}$ H. Lee, M. Chung, N. L. Jeon, Materials Research Society Bulletin, 2014, 39, 51.

${ }^{3}$ K. Haase, R. D. Kamm, Regenerative Medicine, 2017, 12, 285.

${ }^{4}$ S. Hoehme, M. Brulport, A. Bauer, A. Bedawy, W. Schormann, M. Hermes, V. Puppe, R. Gebhardt, S. Zellmer, M. Schwarz, E. Bockamp, T. Timmel, J. G. Hengstler, D. Drasdo, Proceeding of the National Academy of Science, 2010, 107, 10371.

${ }^{5}$ J.M. Munson, A. C. Shieh, Cancer Management Research, 2014, 6, 317.

${ }^{6}$ B. Duan, Annals of Biomedical Engineering, 2016, 45, 195.

${ }^{7}$ P.F. Costa, H.J. Albers, J. E. A. Linssen, H. T. Middelkamp, L. van der Hout, R. Passier, A. van den Berg, J. Malda, A. D. van der Meer, Lab on a Chip, 2017, 17, 2785.

8 J. A. Whisler, M. B. Chen, R. D. Kamm, Tissue Engineering Part C, 2014, 20, 543.

${ }^{9}$ G. S. Offeddu, L. Possenti, J. Loessberg-Zahl, P. Zunino, J. Roberts, X. Han, D. Hickman, C. G. Knutson, R. D. Kamm, Small, 2019, 1902393.

${ }^{10}$ V. S. Shirure , A. Lezia , A. Tao , L. Alonzo and S. C. George , Angiogenisis, 2017, 20,493

11 J. Loessberg-Zahl, A. D. van der Meer, A. van den Berg, J. C. T. Eijkel, Lab on a Chip, 2019, 19, 206.

12 P. Vulto, S. Podszun, P. Meyer, C. Hermann, A. Manz, G. A. Urban, Lab on a Chip, 2010, 11, 1561.

${ }^{13}$ B. Gumuscu, J. G. Bomer, A. van den Berg, J. C. T. Eijkel, Lab on a Chip, 2015, 15, 664.

${ }^{14}$ P. Gribbon, T. E. Hardingham, Biophysical Journal, 1998, 75, 1032.

${ }^{15}$ H. W. Sill, Y. S. Chang, J. R. Artman, J. A. Frangos, T. M. Hollis, J. M. Tarbell, American Journal of Physiology, Heart and Circulatory Physiology, 1995, 268, H535. ${ }^{16}$ R. Sudo, S. Chung , I. K. Zervontonakis, V. Vikerman , Y. Toshimitsu , L. G. Griffith and R. D. Kamm, The Journal of the Federation of American Societies for Experimental Biology, 2009, 23, 2155.

${ }^{17}$ C. Kim, J. Kasuya, J. Jeon, S. Chung, R. D. Kamm, Lab on a Chip, 2015, 15, 301. 



\title{
Chapter 4: Diffusion from Steady State Profile (DSSP) for Low Cost, Low Concentration Characterization of Protein Diffusivity
}

Contributions by: Joshua T. Loessberg-Zahl, Mark. Gillrie, Roger D. Kamm, Albert van den Berg, Andries van der Meer, and Jan C.T. Eijkel

\begin{abstract}
Here we present Diffusion from Steady-State Profile (DSSP), a simple, low cost technique to measure the diffusivity of proteins that are typically used in 3D cell culture. Key to the benefits of this approach is the transformation of a fully developed 2D concentration profile to the time series of a developing 1D profile. As no transient data need be captured, the profile can be imaged with long exposure times. This means that the data is still accessible with lower-end equipment and when lower concentrations of expensive analyte need be used. Analysis is simple as, after transformation of this static $2 \mathrm{D}$ profile to a transient 1D profile, conventional models can be use. To assist with implementation, an extensive diagnostic guide is included for use both as an internal qualitycontrol and for debugging. Furthermore, we walk through the measurement of several common cytokines and report values for the diffusivity of VEGF165 and WNT5a.
\end{abstract}




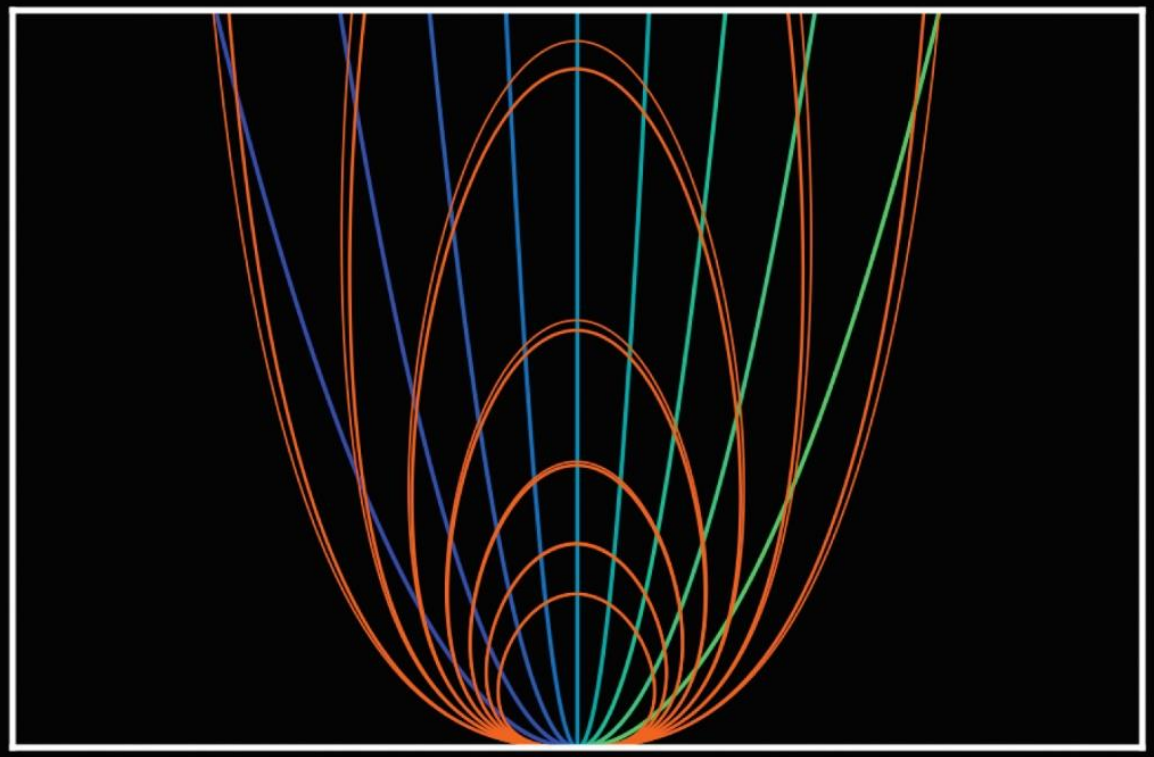




\section{Introduction}

Diffusion of proteins through biological gels has long been of interest in both biological and engineering fields. In tissue engineering, it is important for quantification of the release of growth factors from scaffolds. Specifically, biological hydrogels are used to slow the transport of protein drugs out of the gels by hindering diffusion.[1,2] More recently, diffusive gradients of various cytokines have been shown to possess morphogenic potential. For example, in fetal development, gradients of growth factors in the interstitium were found to determine the local fate of differentiating cells[3,4]. It was also found that growing tumors produce gradients of growth factors to co-opt normal angiogenic processes and encourage vascularization. [5] Experimentally establishing the diffusivity of proteins in hydrogels thus has a broad relevance, but the massive number of possible protein-gel combinations makes an exhaustive library of protein diffusivities next to impossible to compile. Instead, diffusivities are typically either estimated [6] or, ideally, measured in the relevant context.

Hear we propose a new technique, Diffusion from Steady State Profile (DSSP), to establish protein diffusivities. Specifically, DSSP requires tools commonly available to groups working with organ on chip technologies and performing 3D cell culture research. In such works, a 3D cell culture is often established in a hydrogel and often some concentration profile of soluble factors is generated, either a simple gradient, $[7,8]$ or a more complex profile[9,10,11]. Diffusive transport through the matrix is generally used to generate these desired concentration profiles. Unfortunately, the diffusivity of many of the growth factors commonly used in these studies is not well characterized. In these cases, the concentration profile and its time evolution is either estimated using modeling and an approximate value of diffusivity[8] or by using a stand-in fluorescent dextran with a similar molecular weight to the used protein. [12] While this does suffice for a rough estimate, these studies would be improved by a more precise knowledge of the relevant diffusion coefficients. We hope to provide easy access to this information via a simple technique to cheaply determine the diffusivity of an analyite under the same experimental conditions as the use case.

Several techniques have been used to determine the diffusivity of proteins, in various fields of research. Techniques that look at bulk material properties like refractive index [13] have the disadvantage they require concentrations in the range of $1 \mathrm{mg} / \mathrm{mL}$ range, which limits their use to cheap model proteins. In the most common method, the rate at which a protein leaches from a piece of bulk hydrogel is recorded and a diffusion model is fit to 
recover the protein diffusivity in the gel.[1,14] However, this process is quite labor intensive and often requires sampling periods of days to weeks.

More recent techniques require lower concentrations of analyte and can be performed over shorter experimental times. Fluorescence recovery after photo bleaching (FRAP) is commonly used when the protein in question can be easily labeled. $[15,16,17]$ In addition, new microfluidic techniques have been developed. [18] In these modern techniques, the time evolution of a spatial concentration profile is measured and fit to a diffusion model to extract the protein diffusivity. In these techniques, the need to measure a change in concentration profile over time poses a fundamental limit to the needed equipment, concentration of protein and the brightness of the used dye. The signal must be bright enough for the camera to record concentration profiles on time-scales much faster than the characteristic timescale for diffusion in the system. If the time-averaging that comes with longer exposure times approaches the diffusion time, the reported diffusivity is skewed. This can become a serious limitation when expensive high-gain cameras are not readily available and proteins are too expensive to use in higher concentration.

DSSP is a microfluidic method that can be performed quickly, with low concentrations of analyte, and, most importantly, circumvents the core limitations associated with observing concentration change over time. Flow is used to effectively translate time into a spatial dimension, allowing the time series of a diffusing $1 D$ profile to be captured in a static 2D frame. The static nature of the profile means that image acquisition can use as long an exposure time as is required to accumulate sufficient signal.

We demonstrate that DSSP is suitable for commonly used cytokines and only requires tools generally available to the 3D cell culture community. The device design is very simple. Small hydrostatic pressures are used to perfuse a hydrogel-filled y-junction. The analyzed profile develops after this junction when an analyte laden stream meets a stream without analyte. We also provide a clear guide for diagnosing problems with implementation to both direct protocol optimization and develop robust exclusion criteria. Taken together, this work shows that DSSP is not only sufficiently simple to be quickly adopted by labs equipped for 3D cell culture, but also sufficiently robust to produce high quality results with relatively little optimization. 


\section{Results and Discussion}

In this section we will first give a brief overview of the physical system and simplifications of the transport phenomenon therein. Then, we will briefly discuss the procedure to extract diffusivity form the still images, and some practical steps to help diagnose potential problems in implementation. In addition, we provide example measurements in which we measure the diffusivity of fluorescently labeled growth factors, specifically VEGF168 and WNT5a.

\section{Experimental Setup and Protocol}

The critical parts of the setup are intentionally kept as simple as possible. For ease of fabrication, the $y$-channels are relatively large $(0.5 \times 2 \mathrm{~mm})$. The channels are prefilled with an off the shelf fibrin hydrogel. Flow is driven via a small hydrostatic pressure induced by the height of the liquid in wide inlet reservoirs. In addition, all solutions are made in serum containing media to best mimic cell culture conditions.

A cheap and easily bleachable fluorescent tracer, in this case a 40-kDa FITC-Dextran, is included with the analyte to validate the flow conditions and as an internal standard. Note that the fluorophore of the tracer must be chose such that it does not overlap with the signal from the labeled protein so that
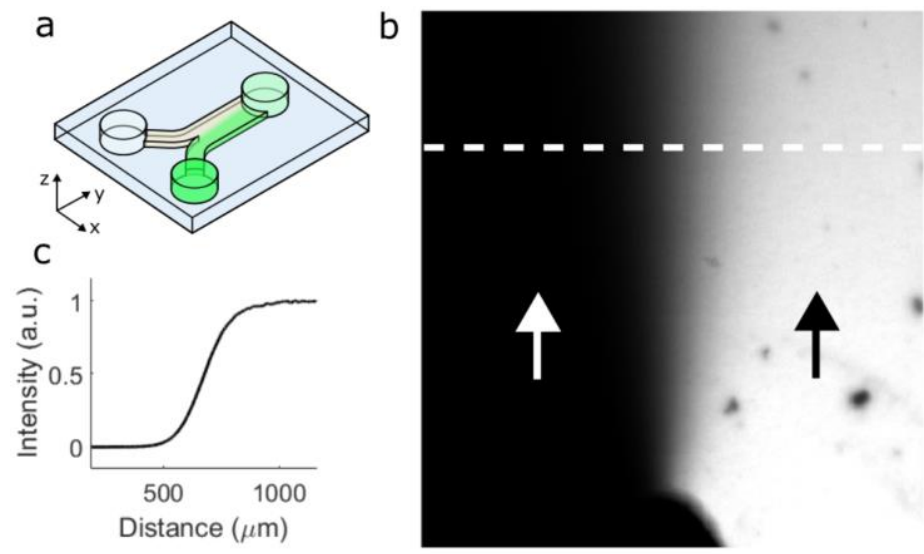

Figure 4.1: (a) schematic of device showing hydrogel filled y-shaped channel with analyte containing media flowed in through the bottom inlet and analyte free media from the top. (b) Fluorescent image of junction showing the developing profile. Arrows indicate the direction of flow. Width of the image is $1.25 \mathrm{~mm}$. (c) Intensity profile taken along dotted line in $b$. 
they may be imaged independently. In our example experiments, FRAP of this tracer is used as a way to determine the flow velocity and as an internal reference. However, if the diffusivity of the dye is well characterized beforehand, the diffusing profile of the tracer can be used to determine the flow velocity without the use of FRAP as is demonstrated in Appendix 2.

After solutions are added to the reservoirs, the flow is allowed time to totally replace the liquid initially residing in the hydrogel. The needed time is calculated via a simple, back of the envelope calculation for travel time [11]. For our setup given with a $3 \mathrm{mg} / \mathrm{ml}$ fibrin gel and a $1 \mathrm{~cm}$ channel length, the linear flow velocity is expected to be $\sim 1.6$ microns per second,[19] indicating that 1.74 hours are needed for the liquid in the channel to be fully replaced by the liquid in the reservoirs. We always allowed 3 hours between filling reservoirs and taking images to give ample time for the profile to develop. At this point the still images of the junction are taken. While all imaging in this work was performed on a confocal microscope, a simple epi-fluorescent microscope with a camera capable of long-exposure would work just as well.

As a final check for strong adsorption with slow kinetics, the device is flushed with analyte free media after the experiment is completed. To do this, the analyte containing media is replaced with analyte free media after through rinsing of the reservoir. Another 3 hours is allowed for the analyte free media to flush through the device and a final image is taken of the junction.

\section{Transport Theory}

The steady state profile used in this technique is generated by introducing an analyte containing stream to a non-analyte containing stream via a y-junction at the inlet of a rectangular microfluidic channel (Figure 4.1a). This rectangular channel is our region of interest. As the two streams flow through the channel, analyte diffuses from the analyte containing stream into the non-analyte containing stream and a steady state concentration profile develops (Figure 4.1b). To make analysis of this 3D profile more manageable we make several assumptions and eventually reduce it to the time evolution of a single $1 \mathrm{D}$ profile.

Primarily, we assume that the profile has reached steady-state. This is guaranteed by allowing sufficient time for the fluid in the device to be totally replaced by the fluid in the reservoirs. Additionally, any binding kinetics between analyte and gel should be fast compared to the time allowed for 
equilibration. This is validated by checking for residual analyte after flushing the device for an equivalent amount of time at the end of the experiment.

Next, we reduce 3D to 2D. All fluid flow occurs in channels pre-filled with a cured hydrogel, thus ensuring Darcy plug flow $[11,20]$. This plug flow means that the complications that arise from analysis of systems with a parabolic flow profile can be avoided.[21] Specifically, any line perpendicular to the height of the channel (z-direction in figure 4.1a) can be assumed to have a constant concentration. Therefore, the steady-state 3D profile can be collapsed along that axis. The resulting $2 \mathrm{D}$ profile can be extracted from a photomicrograph of the profile like the one shown in Figure 4.1b.

We then seek to treat the 2D concentration profile as a 1D concentration profile (Figure 4.1c), developing in time due to diffusion as it translates from junction to outlet. To allow this simplification, the flow velocity is chosen to be high enough such that concentration gradients perpendicular to the flow are much larger than gradients along the flow direction in the region used for measurement. As diffusive flux is proportional to concentration gradient, small gradients in the flow direction imply that diffusion in the flow direction is negligible and only diffusion perpendicular to the flow need be considered. Therefore, a 1D slice of the 2D profile, taken perpendicular to the flow direction, experiences no diffusive flux from its neighboring profiles and can be considered to simply translate downstream while diffusion acts laterally within the profile. The spatial axis parallel to the flow direction can then be directly converted into a time axis with the inverse of the flow velocity as the conversion factor. Profiles taken perpendicular to the flow axis can thereby be converted into the time series of a diffusing 1D profile. See section 2 of Appendix 2 for a more rigorous derivation of this effect.

To ensure that the gradients are negligible in the flow direction, we propose the following criteria:

4.1

$$
\sqrt{\frac{D}{y V}}<1
$$

Here $D$ is the diffusivity of the analyte, $V$ is the flow velocity and $y$ is the distance downstream of the junction. This criteria is met when the rate change in the diffusion length is small compared to the downstream translation rate of the profile. A full derivation can be found in section 1 Appendix 2. 
In our analysis, we treat the diffusing 1D profile as a diffusing step function embedded in an infinite medium, however this requires two additional criteria to be met. First: the width of the diffusing region in the center of the profile must be small compared to the channel width. This is true when:

$4.2 \frac{2}{w} \sqrt{\frac{D y}{V}}<1$

Here $w$ is the channel width. Again, the full derivation can be found in section 1 of Appendix 2.

Second: the most upstream profile in the region used for analysis must take the same form as solutions to the diffusion of a 1D step function. While this seems trivially met if the initial profile created at the junction will be an instantaneous step, this is never actually the case. Effects at the junction caused by development of the flow profile and violation of equation 4.1 always cause distortion of the otherwise instantaneous change in concertation.

However, for our analysis, it is sufficient to show empirically that concentration profiles at the upstream end of the region where equation 4.1 and 2 are met take the same form as the solutions to diffusion of a 1D step function, simply shifted forward by some arbitrary time $t_{\text {off }}[22]$ :

$$
c_{f}=\frac{c_{o}}{2}\left(1+\operatorname{erf}\left(\frac{x}{2 \sqrt{D\left(t+t_{o f f}\right)}}\right)\right)
$$

Here $c_{f}$ is the local un-bound concentration, $c_{o}$ is the maximum unbound concentration, $x$ is the lateral distance from the location of the step change, $t$ is the travel time from the junction, $t_{\text {off }}$ is the offset in time needed to account for heightened diffusion near the junction, and $D$ is the diffusivity. Once $a t_{\text {off }}$ is found to fit for one profile in the range where criteria 1 and 2 are met, all other profiles in that range should follow equation 4.3 with the same $t_{\text {off. }}$ As previously explained, the $t$ coordinate is actually extracted from the $y$ coordinate in the photomicrograph by the conversion $t=y / v$ with $v$ the flow rate.

One noteworthy side effect of our approach is that, at steady-state, binding of the protein to the matrix does not affect the measured value of diffusivity unless concentrations are near saturation. This is opposed to systems that use the time evolution of a diffusing concentration profile (i.e. FRAP), and report an often a slower, "effective" diffusivity. This slowing occurs 
because the diffusing species spends some time bound to the matrix which it would normally spend diffusing. The apparent diffusivity they report is then the free diffusivity multiplied by the fraction of time spent freely diffusing (and not bound) (see Appendix 2 sections 2 and 3 ).

In our technique, when an analyte molecule binds to the matrix it stops diffusing, but also stops translating downstream. While a strongly binding analyte will take longer to move the entire length of the device, it will spend the same amount of time free to diffuse during its journey. Therefore different analytes with different binding but the same diffusivity can be expected to diffuse the same lateral distance for a given downstream distance and DSSP will recover the same diffusivity. For an in depth derivation of this effect and a discussion of how binding near saturation can affect the measurement, see section 2 and 3 of Appendix 2.

\section{Analysis}

To simplify data analysis, we extract a feature of the profile given by equation 4.3 which is strongly and simply related to the diffusivity. For this, we will use the time evolution of the slope at $50 \%$ concentration. From equation 4.3 the time-dependent slope of the curve where $c_{f}=c_{0} / 2$ is

$$
c^{\prime}{ }_{n}=\frac{c_{o}}{2 \sqrt{\pi D\left(t_{n}+t_{o f f}\right)}}
$$

Here $c_{n}^{\prime}$ is the derivative at $x=0$ at time $t_{n}$. From the values of $c^{\prime}$ at two different time points (locations) we can calculate $D$ in terms of the time difference.

$4.5 \quad D=\frac{c_{o}^{2}}{4 \pi \Delta t}\left(\frac{1}{c^{2}}-\frac{1}{c^{2}}\right)$

Here $\Delta t=t_{2}-t_{1}$. Notice that the diffusivity does not depend on $t_{\text {off }}$ any longer and, in fact, $t_{\text {off }}$ need never be explicitly calculated during the analysis. As previously explained, the $t$ coordinate is actually extracted from the $y$ coordinate in the photomicrograph by the conversion $t=y / v$ with $v$ the flow rate. 
To process the images and apply the derived expressions, a series of MATLAB scripts were used. The center line of the profile is first found (Figure 4.2a, dotted line) to account for any slight misalignment of the devices in the microscope, and if needed the image is rotated accordingly. Each 1D slice of the profile perpendicular to this center line is then normalized by the maximum value of intensity in that slice and the slope about the center line is determined. A sample slope-by-time curve thus obtained is shown in Figure $4.2 \mathrm{~b}$. The $y$ axis of the original image has been converted to a time axis simply by dividing the length scale by the flow velocity. At small times (close to the junction) the curve increases before finally realizing the form predicted by equation 4.3. The initial increase in slope is taken to be the effect of the flow development and violation of equation 4.1 near the junction.

To calculate the diffusivity, a time-fixed region is chosen as profile 1 (Figure 4.2b, green band) and the average slope therein is calculated. A second variable region (Figure 4.2b, magenta band) is incrementally swept over the profile. Each time point in this sweep gives a unique $\Delta t$ and $c^{\prime}{ }_{2}$ and thus a unique measure of diffusivity with the resulting curve plotted in Figure 4.2c.
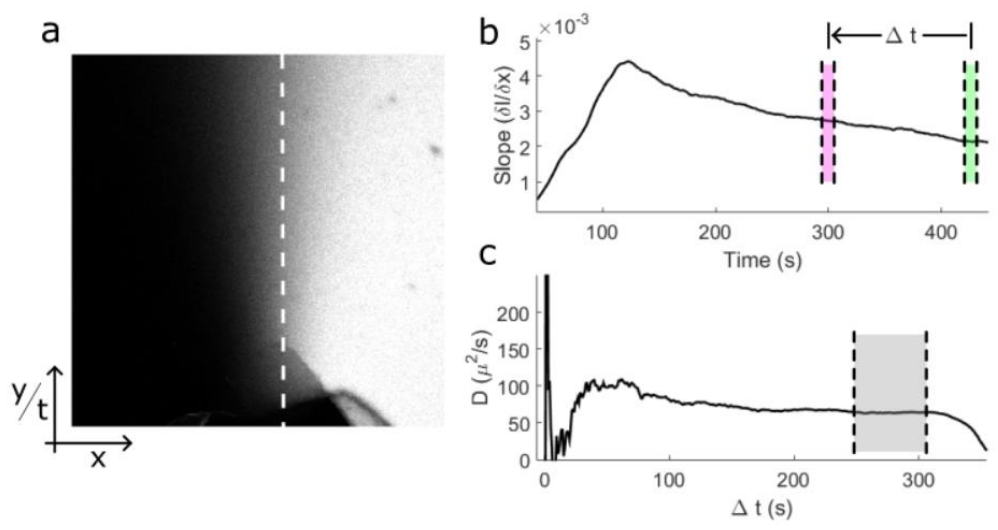

Figure 4.2: (a) Fluorescent image of junction with center line indicated by the dotted line. Note that under the correct flow conditions the $y$ axis of the image is effectively the time axis for the development of the profile shown in Fig1a. Width of the image is $1.25 \mathrm{~mm}$. (b) Slope of profile perpendicular to the center line as a function of distance along the center line, or time. (c) 2-point diffusivity measurement taken from panel $b$ where the first point is taken at the green slice and the second point (magenta slice) is swept over the profile. The reported value of diffusivity is taken from the plateau shown in gray. 
The diffusivity by $\Delta t$ curves can be subdivided into three distinct regions. The highly noisy region seen at small $\Delta t$ is the result of noise in the slope data washing out the true difference between $c^{\prime}{ }_{1}$ and $c^{\prime}{ }_{2}$. When $\Delta t$ is sufficiently large, there is a significant difference between $\mathrm{c}^{\prime}{ }_{1}$ and $\mathrm{c}^{\prime}{ }_{2}$ and the curve plateaus. This is the region in which the reported values of diffusivity are recovered (Figure $4.2 \mathrm{c}$ gray region). Finally, when $\Delta \mathrm{t}$ is too large the slope $\mathrm{C}^{\prime}{ }_{1}$ is significantly affected by the previously mentioned entrance effects and the curve begins to roll off.

\section{Trouble-Shooting}

We also provide a short list of common failure modes of the technique, how to diagnose them and how to solve them (Figure 4.3).

In the first column of figure 4.3, we show an image of a junction where both equation 4.1 and equation 4.2 are violated. Such an image could be due to a number of reasons, the simplest of which being the flow velocity too low. If the flow velocity has been validated and was picked correctly for the expected diffusivity of the analyte, the observed diffuse profile could indicate a problem with sample preparation. Either degradation of the analyte, during handling, presence of a contaminant during staining or the presence of unreacted dye can result in strongly diffuse profile shown. In these cases, the sample may be corrected by re-filtration, but may need to be prepared again from stock if its quality is called into question.

Errors in chip fabrication can also cause problems with the analysis as shown in the second column of figure 4.3. In this example, there are both dust particles in the center line of the profile, as well as a large bubble in the upper right-hand corner. The dust particles cause the dark spots and interfere with the determination of the slope along the center line. While this may be correctable by additional image processing, we found it simpler to take extra care to work dust-free during fabrication. Bubbles present a more difficult challenge for post processing as they prevent true reading of a large area of the profile and may disturb the flow profile locally. We rarely found the presence of bubbles, in case the gel had partially cured before injecting into the device or when gel was injected too quickly. Devices showing defective fabrication were simply rejected. 


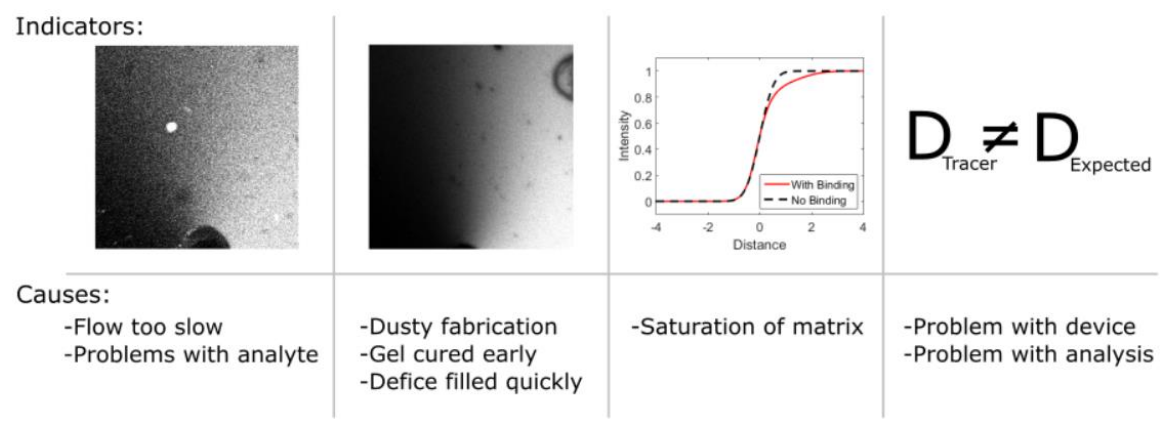

Figure 4.3: Pairs of problem indicators and possible causes for trouble-shooting of DSSP. Column 1 shows a profile where diffusion of the analyte is too fast compared to flow. Column 2 shows a device with bubbles and dust in the channel, column 3 shows a theoretical profile where binding of analyte to the matrix is near saturation. Column 4 covers problems with the internal reference. Width of all microscopy images is $1.25 \mathrm{~mm}$.

Strong binding interaction between the analyte and hydrogel can also be a problem if the concentrations used are near the saturation concentration or insufficient time has been allowed for the binding to reach equilibrium. If concentrations are near saturation concentration a shoulder is expected to appear on the high concentration side of the curve as shown in figure 4.3 column 3. Derivation of the shouldered pro file can be found in Appendix 2. If binding is present but not yet at equilibrium, gradients in the flow direction may be significant, and interfere with the satisfaction of equation 4.1. The presence of strong or slow binding would be indicated by a residual signal remaining in the images taken after wash out of the analyte. If any of these symptoms present, the hydrogel can be replaced by another less adsorbing hydrogel. Additionally, a reagent that competitively binds to the gel can be included with the analyte.

The final quality control is the match between the diffusivity of the tracer and the expected diffusivity. If the tracer is chosen such that its diffusivity is close to the expected diffusivity of the analyte, this final check acts as a catch-all for problems with the particular device and analysis therein. If the match is bad, it could indicate problems caused by of the previously mentioned sources. In addition, it can indicate general disturbance of the flow profile from sources such as delamination of the gel from the walls of the channel or inhomogeneity of the gel. If the recovered diffusivity of the tracer 
is a good match to the expected diffusivity, then the setup and analysis can be trusted and any remaining problems must be specific to the analyte.

\section{Sample Measurements}

As an example of how to implement the technique and apply the exclusion criteria, we performed DSSP on a panel of 10 labeled proteins. For this example, each protein was measured once and each measurement was done in its own unique device. Thus the variation seen here represents both the variation in sample labeling/handling and variation in fabrication from device to device.

Of these 10 devices, 5 were rejected because the measured diffusivity of the tracer differed too strongly from the expected value. This can be seen graphically in Figure 4.4a where the value of diffusivity of the tracer dye recovered via FRAP and DSSP are plotted side by side. Devices in which DSSP differed from FRAP by more than $10 \%$ were rejected. See the trouble shooting section for examples of how the percentage of successful devices may be improved.

We use FRAP as an example of a technique that could be used to validate and debug the implementation of DSSP. However, this can be his can be totally avoided given an optimized dust and bubble free device and a wellcharacterized tracer with molecular weights similar to the analyte. For example, the diffusivity of the labeled dextrans used here could have been
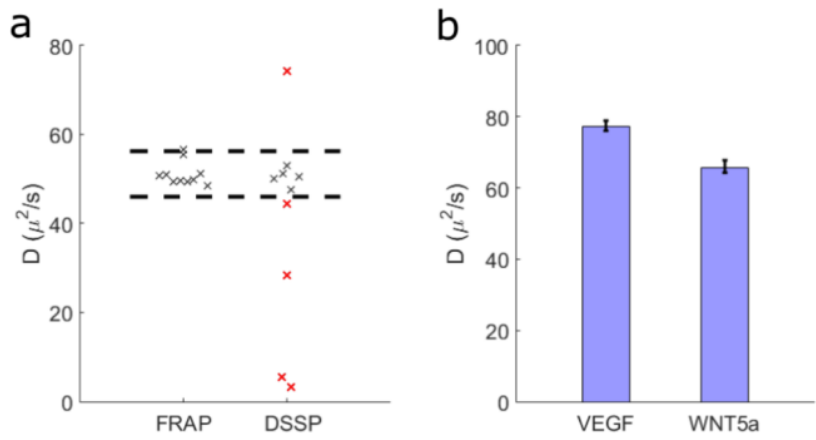

Figure 4.4: (a) diffusivity of tracer measured via FRAP compared to diffusivity of tracer measured via DSSP. Devices in which DSSP differed by more than $10 \%$ from FRAP were rejected (red xs). (b) DSSP values for the diffusivity of VEGF168 and WNT5a. Error bars represent the extents of the data taken from the gray region of Fig2c. 
simply drawn from a number of sources[17]. The only missing piece of information is then the flow velocity, which can be easily recovered from the 2D profile of the tracer as described in section 4 of the Appendix 2.

Of the remaining 5 samples, 3 showed evidence of improper labeling or fragmentation of the protein during handling, either showing overly diffusive profiles (Figure 4.3a) compared to the diffusivity predicted by their molecular weight [15]. These measurements were also rejected, leaving measurements of VEGF 165 and WNT5a. We expect that optimization of the labeling, particularly adding more rinsing and filtration steps, would have yielded a higher number of usable results here.

In this work we did not see any evidence of matrix binding of the proteins used under typical cell culture conditions. None of the measured profiles deviated detectably from the expected form (refer to figure 4.3 column 3) and no residual fluorescent signal was ever detected after flushing the channels with analyte-free media. This was initially puzzling as it is known that VEGF168 can bind strongly to fibrin [23] and that WNT family proteins can have significant interactions with the extra cellular matrix [24]. However, in most studies used to determine matrix binding, the analyte is introduced to the matrix in a serum-free solution. In our work we used serum containing media to better match common 3D cell culture conditions. The relatively high concentration of protein from serum in the media is likely to competitively block any sites available to the relatively low concentration of analyte used both in our work and in 3D cell culture.

The diffusivities of VEGF165 and WNT5a (figure 4.4b) were found to be 77.21 and $65.7 \mu \mathrm{m}^{2} / \mathrm{s}$ respectively. The diffusivity of VEGF165 is fairly well studied and our value is in good accordance with the reported range of literature values $[15,25,26]$ Similarly the value recovered for WNT5a is in the expected range for a globular protein of the same molecular weight. [15]

\section{Conclusions and Outlook}

DSSP allows relatively cheap and robust quantification of the diffusivity of proteins. The extensive internal quality controls detailed here allow easy diagnosis of problems in implementation and ensure that the final results are of a high quality. To demonstrate the efficacy of this approach, we implement a relatively unoptomized setup and measure the diffusivity of a panel of common cytokines. After applying quality controls, our setup still yields values of diffusivity that are a good match for the literature expected 
range, demonstrating the robustness of DSSP and the exclusion criteria detained here.

In fields that apply 3D cell cultures, concentration landscapes of growth factors are becoming more and more complex in both composition and geometry. However, diffusivities of growth factors are still often only measured as needed due to the massive number of potentially useful peptides. As such, there is an increasing need for techniques that can easily validate the transport properties of the used peptides under experimental conditions. We propose DSSP to fill this niche as it is relatively cheap to implement and uses materials and conditions commonly used in 3D cell culture.

\section{Materials and Methods}

\section{Device Fabrication}

Microfluidic devices were made from cast Sylgard 184 PDMS bonded to glass cover slips. The liquid PDMS was mixed at a 10:1 polymer to crosslinker ratio and cast on an acrylic mold. The mold was fabricated by solvent welding thin $\mathbf{5 0 0}$ micron thick pieces of acrylic to a thicker acrylic substrate. The thin pieces of acrylic define the channel geometry in the same way that the SU8 does in a conventional PDMS mold. The thin pieces of acrylic are cut with a Trotec 500 laser cutter.

After demolding all PDMS surfaces were covered with scotch tape to protect them from dust and holes were punched in the PDMS slab to make inlets. The devices and coverslips were then activated in a Harrick oxygen plasma cleaner at high power for 45 seconds and are then bonded by pressing gently together.

Reservoirs were made from plastic $10 \mathrm{~mL}$ syringes with un-threaded male Luer lock tips. The plungers were removed and syringes were cut down so that the first $1.5 \mathrm{~mL}$ above the tip remain. The tips could then be press-fit into the inlets after the devices were filled with hydrogel. If the tips often fall out of the inlets, an extra layer of PDMS with punched holes was added to the bonded device to brace the reservoirs before filling the devices with gel. This slab was $\sim 5 \mathrm{~mm}$ thick and was cut down to be large enough to cover both inlets well but not larger than the area of the device. This slab was then be punched with the same biopsy punch as the inlets at the same spacing of the inlets. Both the bonded device and the slab were then activated in the same plasma 
cleaner and the slab was bonded to the device such that its holes line up with the inlet holes in the device.

If any of the bonding steps fail, the substrates were mechanically cleaned before bonding. This was done by wetting a particulate-free wipe with isopropyl alcohol and then gently rubbing the bonding surface. Before the alcohol could evaporate, the surface was rinsed with ethyl alcohol and before this could evaporate with deionized water. The surface was then blown dry with particulate-free (filtered) compressed air.

\section{Surface Treatment}

In case the gel has trouble adhering to the PDMS surface (as can be evidenced by delamination during perfusion), a surface treatment was applied to the device before filling with hydrogel. This treatment need be applied directly after bonding so that the surface is still clean and activated.

First the chip was filled with a $3 \%(w / w)$ solution of (3aminopropyl)triethoxysilane (Sigma Aldrich) in water. The device was then left to sit at room temperature for 10 min before flushing $3 x$ with water. Next, it was filled with a $10 \%(\mathrm{w} / \mathrm{w})$ solution of glutaraldehyde (Sigma Aldrich) in phosphate buffered saline (Sigma Aldrich). Finally the device was rinsed $3 x$ more times with water before being blown dry. Devices were then filled with hydrogel as normal.

\section{Gel Preparation}

Hydrogels used were $3 \mathrm{mg} / \mathrm{mL}$ fibrin gels. To make the gel, a $6 \mathrm{mg} / \mathrm{ml}$ solution of fibrinogen (Sigma Aldrich) in Phosphate Buffered Saline (PBS) (Sigma Aldrich) was prepared by mixing the dry fibinognogen with the PBS and heating the mixture to 36 degrees Celsius for 30 min until the fibrin dissolved. After dissolution the solution is chilled on ice. A $4 \mathrm{U} / \mathrm{mL}$ thrombin (Sigma Aldrich) in Endothelial Growth Media (EGM) (Sigma Aldrich) solution was then also prepared on ice. Finally the solutions are mixed 1:1 and injected into the waiting device. The device was transferred immediately to a humidity chamber [27] and placed in an incubator at 36 degrees Celsius to cure for at least $10 \mathrm{~min}$. After curing, inlet syringes were added.

The gel forming protocol used here was based off of 3D culture work by the group of Roger Kamm [27], but should be adapted to the relevant experimental conditions. 


\section{Protein Labeling}

On microgram of recombinant human WNT5A (R\&D Systems) and VEGFA-165 (Peprotech) were labeled using the Alexa Fluor ${ }^{\circledR} 568$ NHS (Succinimidyl Ester, Life Technologies) as per the manufacturer's guidelines for 1 hour at room temperature. Reactions were quenched with $100 \mathrm{mM}$ glycine at room temperature for 30 minutes. Glycine-conjugated and unreacted dye was removed by washing $3 \mathrm{x}$ with PBS using $3 \mathrm{kDa}$ Amicon microfuge filters (Millipore Sigma). Protein solutions were stored at -80 in PBS until use.

\section{Perfusion Experiments}

After the analytes were labeled, they were diluted to 100 nanograms per $\mathrm{mL}$ in EGM with 20 microgram per $\mathrm{mL}$ of a $40 \mathrm{kDa}$ FITC dextran (Sigma Aldrich). $1 \mathrm{~mL}$ of the final solution was added to one inlet of the prepared device, and the other inlet was filled with analyte free EGM. The devices were then left in the incubator at 36 degrees Celsius for 3 hours to let the concentration profile reach steady state.

One by one, the devices were next taken to the microscope for imaging. In this case we used an Olympus FV1000 but any normal epiflourescent microscope equipped with the correct filters and light source should be sufficient. In our experiments we then performed a FRAP experiment on the tracer analyte, downstream of the inlet to internally validate both flow velocity and diffusivity of the dextran. This step was included as a sanity check and may be skipped if the diffusivity of the analyte is well known and the fabrication protocol is robust. If it is skipped the velocity may be recovered via the technique detailed in section 4 of the Appendix 2 .

Finally the diffusing profile of both tracer and analyte was imaged.

\section{DSSP Analysis}

Before DSSP analysis, the image of the junction was pre-processed. For each horizontal line of the image, the asymptotic value of the low concentration end of the profile was subtracted from the entire profile, then the entire profile was divided by the asymptotic value of the high intensity side of the profile.

Next the center line of the full 2D profile was found. To do this, two dust-free cross sections are chosen far downstream of the junction in the region where equations 4.1 and 4.2 simultaneously apply. The midpoint of the profile was calculated for both as the point at which the intensity is at half of 
the maximum. The center line of the 2D profile was taken as the line between the two midpoints.

The slopes used in equation 4.5 were the slopes of the intensity profile perpendicular to this line. These slopes were determined by fitting a line to the linear region of the profile. A detailed discussion of the mathematics used to extract diffusivity from these slopes can be found in the Results and Discussion section and Appendix 2.

\section{FRAP Analysis}

The FRAP data was analyzed according to M. P. Jonsson, et al. [28] using their software to extract both diffusivity of the tracer and the flow velocity. 


\section{References}

1 J. B. Leach, C. E. Schmidt, Biomaterials, 2005, 26, 125.

${ }^{2}$ T. R. Hoare, D. S. Kohane, polymer, 49, 1993.

${ }^{3}$ B. G. Chung, L. A. Flanagan, S. W. Rhee, P. H. Schwartz, A. P. Lee, E. S. Mounki, N. L. Jeon, Lab on a Chip, 2005, 5, 401.

${ }^{4}$ J. A. Burdick, G. Vunjak-Novakovic, Tissue Engineering Part A, 2009, 15, 205.

${ }^{5}$ E. T. Roussos, J. S. Condeelis, A. Patsialou, Nature Reviews Cancer, 2011, 11, 573.

${ }^{6}$ M. E. Young, P. A. Carroad, R. L. Bell, Biotechnology and Bioengineering, 1980, 22, 947.

${ }^{7}$ S. Bersini, J.S. Jeon, D. Dubini, C. Arrigoni, S. Chung, J. L. Charest, M. Moretti, R.D. Kamm, Biomaterials, 2014, 35, 2454.

${ }^{8}$ G. S. Jeong, S. Han, Y. Shin, G. H. Kwon, R.D. Kamm, S. Lee, S. Chung, Analytical Chemistry, 2011, 83, 8454.

${ }^{9}$ B. M. Baker, B. Trappman, S. C. Stapleton, E. Toro, C. S. Chen. Lab on a Chip, 2013, 13, 3246.

${ }^{10}$ S. G. M. Uzel, O. C. Amadi, T. M. Pearl, R. T. Lee, P. T. C. So, R. D. Kamm, Small, 2017, 12, 612.

11 J. Loessberg-Zahl, A. D. van der Meer, A. Van den Berg, J. C. T. Eijkel, Lab on a Chip, 2018, 19, 206.

${ }^{12}$ R. Sudo, S. Chung, I. K. Zervantonakis, V. Vikerman, Y. Toshimitsu, L. G. Griffith, R. D. Kamm, The FASEB Journal, 2009, 23, 2155.

${ }^{13}$ S. Liang, J. Xu, L. Weng, H. Dai, X. Zhang, L. Zhang, journal of controlled release, 2006, 115, 189.

${ }^{14}$ B. Narsimhan, S. K. Mallpragada, N. A. Peppas, Release Kinetics, Data Interprestation, E. Mathiowitz (Ed.), Encyclopedia of Controlled Drug Delivery, Wiley, New York (1999), 321-305.

${ }^{15}$ D. A. Berk, F. Yan, M. Leunig, R. K. Jain, Biophysical Journal, 1993, 65, 2428.

${ }^{16}$ E. M. Johnson, D. A. Berk, R. K. Jain, W. M. Deen, Biophysical Journal, 1995, 1561.

${ }^{17}$ P. Gribbon, T. E. Hardingham, Biophysical Journal, 1998, 75, 1032.

${ }^{18}$ M. H. Hettiaratchi, A. Schudel, T. Rouse, A. J. Garcia, S. N. Thomas, R. E. Guldberg, T. C. McDevitt, APL Bioengineering, 2018, 2, 026110.

${ }^{19}$ M. A. Swartz, M. E. Fleury, Annual Review Biomedical Engineering, 2007, 9 229.

20 J. R. Levick, Quarterly Journal of Experimental Physiology, 1987, 72, 409.

${ }^{21}$ A. E. Kamholz, B. H. Weigl, B. A. Finlayson, P. Yager, Analytical Chemistry, 1999, 71, 5340.

22 J. Crank, The Mathematics of Diffusion, Clarendon Press, Oxford, 1976. 
${ }^{23}$ A. Sahni, C. W. Francis, Blood, 2000, 96, 3772.

${ }^{24}$ G. T. Wong, B. J. Gavin, A. P. McMahon, Molecular and Cellular Biology, 1994, 14, 6278.

${ }^{25}$ A. Kohn-Luque, W. de Back, Y. Yamaguchi, K. Yoshimura, M. A. Herrero, T. Miura, Physical Biology, 2013, 10, 066007.

${ }^{26}$ T. Miura, R. Tanaka, Mathematica Models of Natural Phenomenon, 2009, 4, 118.

${ }^{27}$ G. S. Offeddu, L. Possenti, J. Loessberg-Zahl, P. Zunino, J. Roberts, X. Han, D. Hickman, C. G. Knutson, R. D. Kamm, Small, 2019, 1902393.

${ }^{28}$ P. Jonsson, M.P.Jonsson, J. O. Tegenfeldt, F. Hook, Biophysics Journal, 2008, 95, 5334 . 



\title{
Chapter 5: Chip holder-integrated Pneumatic Logic for Modular Multiplexing of Microfluidic Devices
}

Contributions by: Joshua Loessberg-Zahl, Albert van den Berg, Andries van der Meer, and Jan C.T. Eijkel

\begin{abstract}
Here we present a new solution to the problem of microfluidic multiplexing. We have designed and tested a 3D printed pneumatic controller which can be easily built into chip holders for microfluidic devices. This in-chip holder design generally reduces the amount of interfacing needed and can interface with many different microfluidic devices making it modular. Our technique leverages the advantages of both 3D printed valves and clamped gasket valves. Pressure can be easily routed in 3D inside of 3D printed blocks, but these blocks have no difficult-to-vacate dead end channels because all channels terminate at a clamped elastomeric membrane which serves as the actuated element. Impregnation of the elastomeric membrane makes our valves ideal logical elements: They are both leak free when closed and biased towards the closed state. To demonstrate the potential of this technology for multiplexer design, we built and tested a simple shift-register based multiplexer in a chip holder. In the tested configuration, our valves were shown to operate well up to 20 $\mathrm{Hz}$, and state storage elements were able to hold their state for $>24 \mathrm{hrs}$.
\end{abstract}


D) 


\section{Introduction}

Parallelization of microfluidic devices is of great value to microfluidic applications in which an increase of throughput is desirable.[1,2] For example, cell culture applications often require the dosing of specific cell culture chambers with specific cocktails of chemicals. The ability to independently control the chemical makeup of media in multiple chambers at once dramatically improves experimental throughput. [3] In chemistry, microfluidics allows specific reactions to be performed with a high degree of environmental control and small volumes of reagents. Here again the number of simultaneous and independent reactions that can be run dramatically improves the rate of testing.[4] In general, when microfluidics is used for sample analysis, parallelization of reactions is of great value. In spite of the benefits, parallelization has been a persistent challenge in microfluidics and is often achieved in a highly application specific and expensive manner.

Form a design complexity perspective, the simplest way to multiplex a given device is to make many independent copies of the device and use them all in parallel. This, however, often results in highly complicated interfacing as each device requires its own individual connections. Macro scale pumps used to drive the device must also be copied per device.[5] Existing systems for parallelization like pipetting robots are sometimes used to automate this approach, but these can be prohibitively expensive and are not optimized for microfluidics.[6]

To address the interfacing problem, valve-based multiplexers are often implemented within devices. These allow a few inputs to control a large number of independent channels within the device. [7] However, these multiplexers often require complex and failure prone multilayer fabrication processes, even when the device being multiplexed is relatively simple. Furthermore, these multiplexers often only allow specific combinations of outputs to be addressed at once and therefore require redesign per application.[7,8,9]

For a limited set of applications, droplet-based multiplexers are used. $\left[{ }^{10},{ }^{11}\right]$ These work well when: the desired process can be carried out in a droplet, an optical readout is sufficient for analysis, and the specific starting conditions of each droplet are not important. $[10,11,12,13]$ Reading out data through chemical or electrical interactions on the other hand requires making 
physical contact with the liquid inside the droplets. This is often difficult as ease of droplet transport requires that their chemistries be designed to explicitly prevent droplets from wetting the surfaces.

In this work, we present a chip holder- integrated multiplexer for parallelizing microfluidic processes that circumvents many of the mentioned problems. With our chip holder, we then show we can automate the most generally multiplexed process in microfluidics: the dosing of liquid. Much like in-device multiplexers it drastically reduces the amount of tubing required to operate devices. The demonstrated approach however promises substantial cost reductions. Keeping the multiplexing separate from the microfluidics means that the (often disposable) microfluidic devices can be kept simple while the relatively complicated multiplexer only need to be fabricated once. Furthermore, unlike other off-device approaches to multiplexing like pipetting

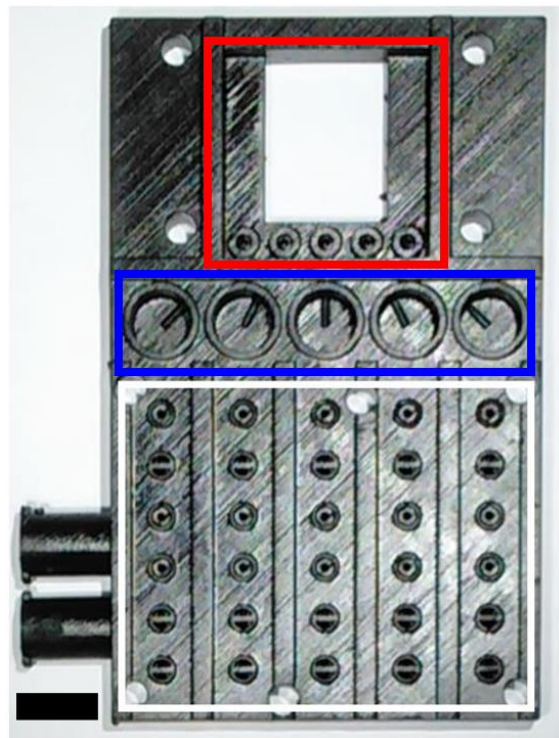

Figure 5.1: Photograph of the in-chip holder multiplexer. The white rectangle outlines the valve containing area in which all logical operations are per formed. The blue rectangle outlines the reservoirs which can be pressurized by the multiplexer to pump liquid into an attached device. The red square outlines the area in which a microfluidic device may be placed with its inlets aligning with the ports at the bottom of the square. Luer-lock inlets are at the bottom right of the chip holder. Scale bar (left bottom) is $0.5 \mathrm{~cm}$. 
robots or parallel pumps our chip holder is inexpensive, small in form factor, and designed to easily interface with microfluidic devices.

The chip holder we demonstrate here combines the advantages of 3D printed valves and clamped elastomeric valves. Like other clamped elastomeric valve designs, we use an elastomer membrane as the actuated element in the valve. [14] The valve is defined by the geometry of the two stiff blocks that clamp this membrane. These two pieces of plastic are referred to henceforth as "the blocks". However, our use of 3D printing for the blocks means that we can easily route pressure in $3 \mathrm{D}$ through channels within the

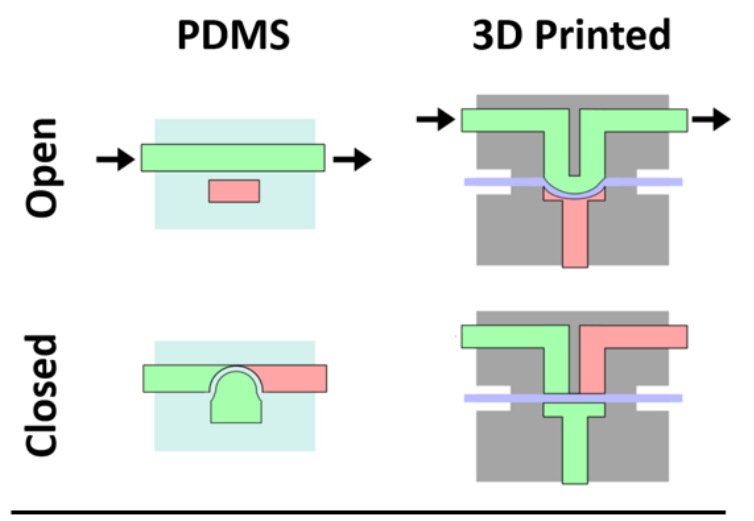

\section{Control}

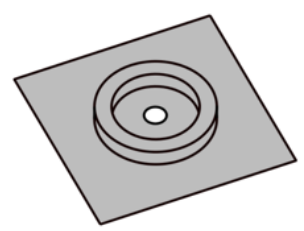

Switched

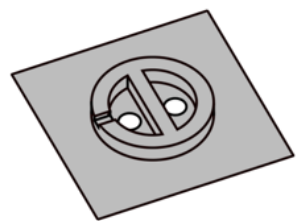

Figure 5.2: Top panel compares the typical PDMS valves to the valves demonstrated here. In the case of the PDMS valves two channels in PDMS are separated by a thin membrane of the same material. In our valves, two stiff 3D printed blocks clamp a silicone gasket. In both cases the thin elastomer between the two halves is actuated by pressure in one side to prevent flow through the other. Green represents a channel with high internal pressure, red represents a channel with low internal pressure. Arrows indicate flow. Bottom panel shows the design of the two halves of our valve in 3D. Inlets are shown in white. While not all valves have a designed leak, the switched valve here has one near its left inlet. 
blocks without the need for complex machining or bonding. Furthermore, unlike 3D printed valve designs without discrete membranes, evacuation of resin from the printed cavities is not a problem for our technique.[15,16]

Our use of a dodecane-impregnated membrane allows us to make leak free valves that are operated using positive pressure. The impregnation also significantly biases our valves towards the closed state, meaning that large pressures can be switched by relatively small ones. While some other valve designs also have this property, our design requires significantly simpler fabrication. $[17,18,19,20]$ This bias allows us to make complex devices similar in design to transistor-based electronics, but without the need to use complex resistor networks to precisely control the pressure. Following the electronics analogy, we can realize many different transistor-based multiplexers with our valves. The multiplexer demonstrated in this paper is effectively a shift register in which a signal input and a clock input can set the state of an arbitrary number of outputs (Figure 5.1).

\section{Design and Manufacturing}

\section{Valve Design}

The valves in our system are essentially a new take on clamped gasket valves.[14] These clamped gasket valves are differentiated from the typical PDMS based valves by the fact that a simple membrane is the only elastomeric component. (Figure 5.2, top) In our case the membrane is an unmodified, offthe-shelf piece of silicone gasket material. All the valve defining geometry is located on rigid pieces of 3D printed plastic that clamp the membrane. The switched side of our valves has two inlets separated by a narrow wall and the control side has only one inlet. (figure 5.2 bottom) When pressure is applied to the control side, the gasket is pushed tightly against the wall of the switched side and no fluid can flow to the outlet of the switched side.

Our valves are biased towards the closed state and leak free when closed. This means that relatively little pressure is required at the control side of the valve to keep the switched side totally shut. Valves with this property are highly desirable as logic elements. Otherwise, relatively complicated resistor networks are required to ensure that the pressure on the switched side of the valve is always lower than the pressure on the control side.

In our devices, the bias is enhanced by the impregnation of the silicone gasket. Impregnation is achieved by pre-soaking the gasket in dodecane for 30 
min before assembly of the chip holder. The gaskets are then dried with a particulate-free cloth before being clamped in place. Some of the dodecane remains adsorbed in the gasket and, when the gasket is compressed, a small amount is squeezed out onto the surface. The dodecane on the surface of the gasket wets both the gasket and the plastic block and thus creates an air-tight seal. We believe that it is the capillary force between the dodecane, the gasket and the block that provides both the bias and the leak free quality of our design. Use of unimpregnated membranes always resulted in leaky, unbiased valves.

While the leak-free nature of our valves is generally an advantage, it can also pose a problem if the output of the switched side of a valve leads to a dead end (i.e. the control side of another valve). Pressurized air can be trapped in these dead ends while often the desired effect for the pressure on the outlet of a valve is to return to ambient when the valve is closed. This is solved simply by including designed leaks in the valves as shown in figure 5.2 bottom. In valves where trapping of outlet pressure is desirable (i.e. valves used for data storage) the leaks are excluded.

\section{Design Layers}

While each block is fabricated as one unit, they are designed in three parallel layers. (Figure 5.3)

The lowest layer is the valve layer. This layer includes raised ridges of two heights. Lower ridges are designed to locally clamp the gasket and define the previously mentioned valve geometry. Higher ridges act as mechanical stops to physically set the distance between the blocks and set the degree of gasket compression. Higher ridges are therefore included in areas where no gasket is present and directly contact high ridges on the opposing block. All designed leaks in the valves vent into the area between the gasket and the blocks.

The next layer is the logic routing layer. This layer contains all pneumatic routing between the valves in the valve layer. It is by way of this routing that the output of one valve may actuate the control side of another. Pneumatic routing is done via channels through the plastic block. These pneumatic channels rise out of valve layer, travel laterally in the logic control layer, then return down to the valve layer. Channels from the power layer (see next section) to the valve layer also travel through this layer. 


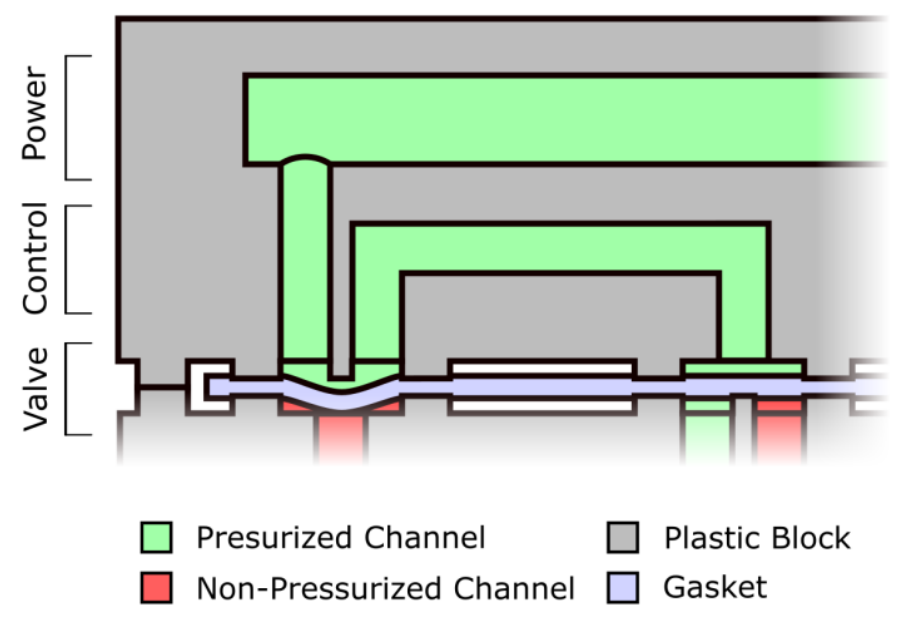

Figure 5.3: Schematic of the different layers of the design. All of the geometry mentioned in figure 5.2 as well as mechanical stops to set the spacing between the blocks are found in the valve layer. All channels that route pressure between valves are found in the control layer and the always pressurized larger channels that supply pressure to the system are found in the power level.

The power layer contains larger channels which bring pressurized air to all valves that need continuous pressure. The goal of this layer is to supply an identical pressure across the entire chip holder. If the difference in pressure from valve to valve is greater than the bias of the valves, it may no longer be possible for the pressure output from one valve to successfully close another. The channels in the power layer are therefore designed with the largest diameter of all the channels in the chip holder to have the smallest resistive pressure drop over their length.

\section{Clamping Features}

To ensure proper functioning of the valves they must be placed in close proximity to both mechanical stops and clamping bolts. Proximity to clamping bolts is important as any concave bowing of the block will cause insufficient clamping of the gasket and the bolts provide the force to correct this. Similarly, convex bowing of the block will cause the gasket to be clamped too tightly. Proximity to mechanical $s$ tops prevents this as the stops enforce the correct clamping depth. In our designs, valves are always within $1 \mathrm{~cm}$ of a mechanical stop and within $3 \mathrm{~cm}$ of a clamping bolt. 
To ensure that spacing criteria are met, we arrange the valves in rows as shown in figure 5.1. Each row of valves has its own gasket and is separated from the other rows by a mechanical stop. Bolt holes are regularly spaced throughout the design.

\section{Interfacing}

To interface with external pressure sources, Luer-lock connectors are included on the periphery of the device as can be seen in figure 5.1. These side connectors directly connect to channels in the logic or power layer of the design.

Interfacing with microfluidic devices can be done via the smaller outlet ports in the red box of figure 5.1. The microfluidic devices should be designed such that its inlets align with these ports. A gasket with corresponding holes punched in it is included between the inlets of the microfluidic devices and the outlets of the chip holder. When the clamping bolts are tightened, the gasket is compressed, making the interface leak free, but allowing fluid to flow from the chip holder to the microfluidic device through the punched holes.

\section{Implementation and Testing}

To show the feasibility of this approach, we designed and tested a shift register built into a chip holder. The physical device is shown in figure 5.1. A schematic of the circuit is shown in figure 5.4. In our design the pressurized state of a given channel represents a logical 1 and the depressurized state represents a logical zero. The design consists of a chain of D-Latches in which the output of each latch is connected to the input of the next. The input of the first latch is the signal input of the register. A common clock signal is introduced via the clock input and simultaneously drives the clock input of all of the latches. As this input cycles from low to high, each latch passes its output state to the next latch, causing data to move down the chain.

In this configuration, a single time-delineated data input can be used to determine the state of the outlets. The time-delineated data signal is introduced to the signal inlet while a square wave is fed into the clock inlet. (figure 5.5, left). Once the data signal is finished, the clock is left in the low pressure state and the states of the latches in the chain reflect the states of the data signal over time. The state of each latch in the chain is used to determine the pressure of an outlet and the state of the outlets will be maintained until the clock cycles again. 
In this work we have built a 5-bit shift register, and thus we input five pulse long clock and data signals to set the values of the outputs. Several sample signals are shown in figure 5.5 left. To better visualize the state of the outputs in order to demonstrate that the shift register works, the outputs were connected to test tubes filled with died water. In this configuration, bubbling in the tube and an elevated liquid level indicate that pressure is applied and air is flowing out of the respective output of the register. For a given input signal shown in the left panel of figure 5.5, the corresponding outputs are indeed

a)
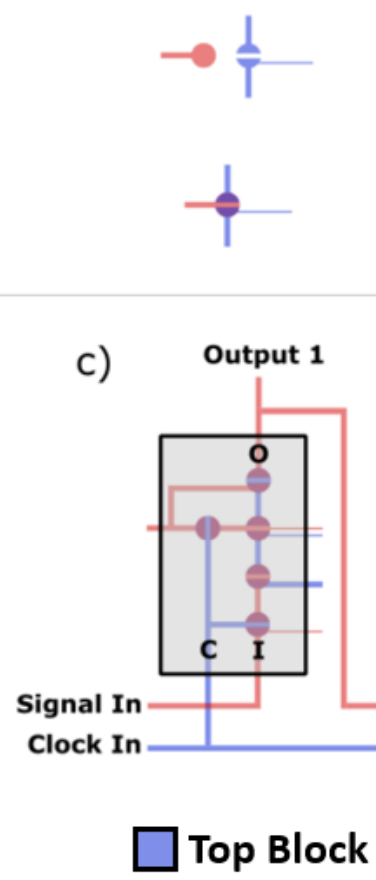

Clock In

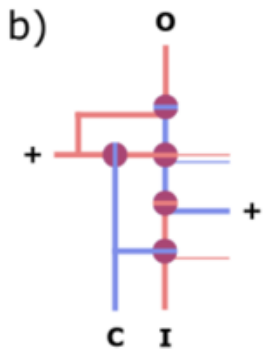

C I

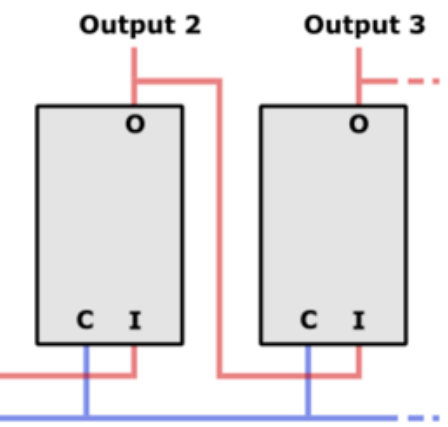

Figure 5.4: Schematic representation of the shift register design. Features from the control and valve layers are shown. Features in the top block are shown in blue while features in the bottom block are shown in red. Panel a demonstrates our schematic representation of the valves. Circles represent the control side of a valve, split circles represent the switched side of a valve. Thick lines represent channels in the control layer, thin lines represent controlled leaks from the valve into the space between valve and gasket. Connections to the power layer are indicated by a + . Panel $b$ shows a schematic of the $D$ latch used to implement our shift register. C indicates the clock input of the latch, $\mathbf{I}$ indicates the data input and $\mathbf{O}$ indicates the output. Panel $\mathrm{c}$ shows how the $\mathrm{D}$ latches are connected to make a shift register. 


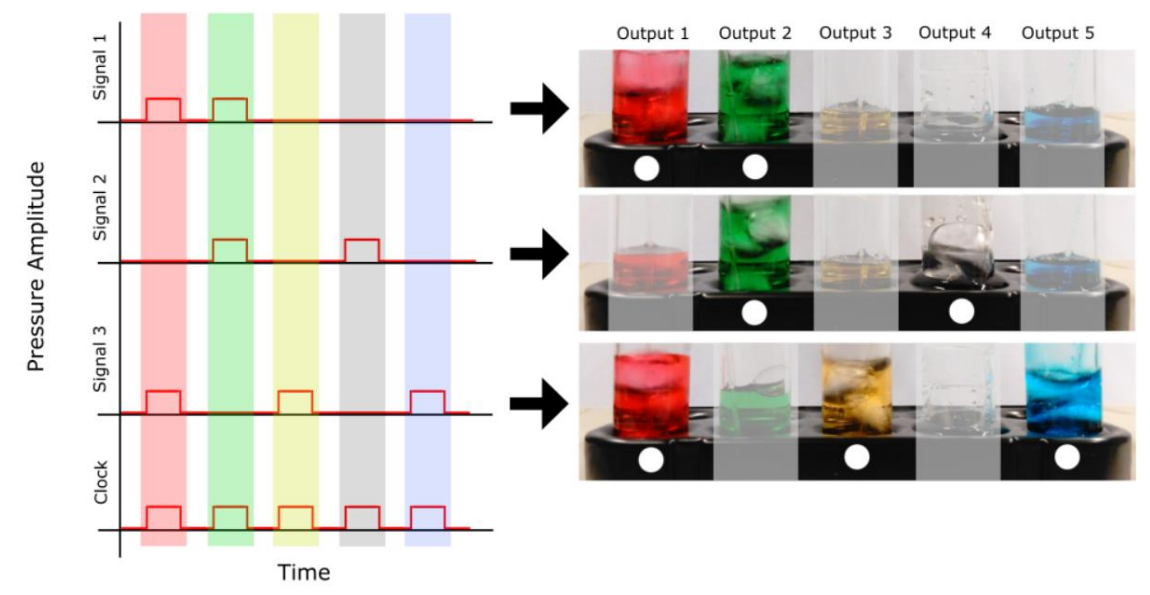

Figure 5.5: Data inputs and resulting outputs of the in-chip holder multiplexer. Left panel shows the data input into the chip holder over time. Each row shows a different data signal. The clock signal shown in the last row was the same for all data signals. The state of the outputs of the shift resister after applying the indicated input signal is shown on the right-hand side of the figure. In this experiment each output of the shift register is connected to the bottom of its own vial. Active outputs are actively bubbling while inactive outputs are still. Active outputs are indicated by a white dot to guide the eye while inactive outputs are grayed out.

bubbling in the right panel, demonstrating the proper functioning of the multiplexer.

For the design shown here the maximum frequency of actuation was $20 \mathrm{~Hz}$. When the frequency increased above this threshold, the state of the outputs no longer reflected the input signal. In this failure case, some of the bits from the input signal are missing or incorrect. We hypothesize that this occurs when the clock frequency is faster than the switching time of the latches, but more work is required to be sure of the specific cause.

\section{Future Work and Conclusions}

\section{Work Remaining}

Before our work can be considered a successful proof of concept of an in-chip holder multiplexer, we must demonstrate that it can interface with microfluidic devices without leaks and that it can dispense liquid into the attached devices at a reasonable flowrate. The current design has a set of reservoirs designed to hold aqueous solutions of choice to dispense into an 
attached microfluidic device.(figure 5.1) However, in the current prototype chip holder, the 2 bar driving pressure empties the reservoir in a matter of seconds. This has made it nearly impossible to test the interfacing and liquid dispensing capabilities of the design, but should be correctable either by adjusting the flow resistance of the channels between the reservoirs and the outlets or by adding a microfluidic device with a high flow resistance.

\section{Conclusions}

We have shown that the often cumbersome task of multiplexing microfluidic devices can be surmounted by including a multiplexer made from clamped gasket valves within a chip holder. With the demonstrated design, many parallel microfluidic devices may be independently controlled with only two input signals.

The novel use of impregnated gaskets to make leak-free and biased valves dramatically eases circuit design constraints common to working with elastomeric valves. Explicitly, the ability to switch large pressures with smaller ones removes the need for precisely tuned resistor networks to supply ever decreasing pressure to the switched side of the valves. The number of valves used in a device can therefore be scaled up dramatically with little design overhead.

\section{Outlook}

The simple multilayer design used here can facilitate the translation of many different kinds of digital electronic circuits into pneumatic devices. For example, digital to analogue controllers would be capable of turning a series of on or off pneumatic inputs into a variable pressure or variable flow outputs. Similarly, programmable state machines, as have been demonstrated in PDMS devices, could be integrated for in-chip holder process automation. Ultimately integration of multiplexing and various other functions into a single chip holder could virtually remove the need for microfluidic peripherals.

\section{Materials and Methods}

\section{Chip holder fabrication}

The chip holder shown here was printed with black resin V2 on a Form2 3D printer (Formlabs). Layer height was set to the finest resolution (25 microns). Parts were always printed such that no channels were in a plane parallel to the build platform. This was achieved by orienting the parts such 
that they stood on one corner. Supports for printing were generated automatically with the Preform software (Formlabs)

Membranes were $0.2 \mathrm{~mm}$ thick silicone gaskets (Acme Rubber). Gaskets were first soaked in dodecane for $30 \mathrm{~min}$ to impregnate them. Gaskets were then dried with a particulate free wipe till no more oil was apparent on the surface. After impregnation gaskets were cut to the appropriate size to fit between mechanical stops while still covering the other features. Holes for interfacing were punched through the gasket with a $1 \mathrm{~mm}$ biopsy punch.

\section{Use Protocol}

Before use, any liquids that need be dispensed were added to the reservoirs in the chip holder. All gaskets were then placed carefully to cover all reservoirs, valves, and chip interfacing while leaving the mechanical stops uncovered. If a microfluidic chip was being tested, the chip to be controlled was then added into the chip holder and the second block of the chip holder was put in place. If no chip was used, tubing was connected between the outlets and the vessels to be pressurized before putting the second block of the chip holder in place. Clamping screws were tightened until any gaps between mechanical stops were seen to disappear.

The data and clock inputs of the chip holder were then each attached to a computer controlled valve (Festo) with TYGON tubing. (TYGON) The power inputs were connected to a static pressure regulated input, also with TYGON tubing. All pressures used here were 2 bar. The chip holder was then ready to use. For our 5 bit shift register a five pulse clock signal was generated simultaneously with a 5 pulse data signal to set the state of the outputs. Festo valves were controlled and signals were generated using a MATLAB script. 


\section{References}

${ }^{1}$ S. Vyawahare, A. D. Griffiths, C. A. Merten, Chemistry and Biology, 2010, 17, 1052.

2 J. Melin, S. R. Quake, Annual Review of Biophysics and Biomolecular Structure, 2007, 36, 213.

${ }^{3}$ M. Mehling, S. Tay, Current Oppinion in Biotechnology, 2014, 25, 95.

${ }^{4}$ P.S. Dittrich, A. Manz, Nature Reviews, 2006, 5, 210.

${ }^{5}$ C.K. Fredrickson, Z. H. Fan, Lab on a Chip, 2004, 4, 526.

${ }^{6}$ V. van Duinen, A. van en Heuvel, S. J. Trietsch, H. L. Lanz, J. M. van Glis, A. J. van Zonneveld, P. Vulto, T. Hankemeier, Scientific Reports, 2017, 7, 18071.

${ }^{7}$ T. Thorson, S. J. Marekl, S. R. Quake, Science, 2002, 282, 580.

${ }^{8}$ G. A. Cooksey, C. G. Sip, A. Folch, Lab on a Chip, 2009, 9, 417.

${ }^{9}$ I. E. Araci, P. Brisk, Current Opinion in Biotechnology, 2014, 25, 60.

${ }^{10}$ L.F. Cheow, L. Yobas, D. Kwong, Applied Physics Letters, 2007, 90, 054107.

${ }^{11}$ A. B. Theberge, E. Mayot, A. E. Harrak, F. Kleinschmidt, W. T. S. Huck, A. G. Griffths, Lab on a chip, 2012, 12, 1320.

12 L. Rosenfeld, T. Lin, R. Derda, S. K. Y. Tang, Microfluidics and Nanofluidics, 2014, 16, 921.

${ }^{13}$ Y. Zhu, Q. Fang, Analytica Chimica Acta, 2013, 787, 24.

${ }^{14}$ I. R. G Ogilvie, V. J. Sieben, B. Cortese, M. C. Mowlem, H. Morgan, Lab on a Chip, 2011, 11, 2455

${ }^{15}$ A.K. Au, N. Bhattacharjee, L.F. Horowitz, T.C. Chang, A. Folch, Lab on a Chip, 2015, 15, 1934.

${ }^{16}$ H Gong, A. T. Woolley, G. P. Nordin, Lab on a Chip, 2016, 16, 2450.

17 J.A. Weaver J. Melin, D. Stark S. R. Quake, M. A. Horowitz, Nature Physics, 2010, 6, 219.

18 J. Y. Baek, J. Y. Park, J. I. Ju, T. S. Lee, S. H. Lee, Journal of Micromechanics and Microengineering, 2005, 15, 1015.

${ }^{19}$ B. Mosadegh, H. Tavana, S. C. Lesher-Perez, S. Takayama, Lab on a Chip, 2010, 11, 738.

${ }^{20}$ N.S.G.K Devaraju, M. Unger, Lab on a Chip, 2012, 12, 4809. 


\title{
Chapter 6: Voltage Mediated Delamination of Suspended 2D Materials Explains Commonly Observed Breakdown
}

\author{
Contributions by: Joshua T. Loessberg-Zahl, Douwe S. de Bruijn, \\ Wesley T.E. van den Beld, Edwin Dollekamp, \\ Eldad Grady, Ashok Keerthi, Johan Bomer, \\ Boya Radha, Harold J.W. Zandvliet, Ageeth A. Bol, \\ Albert van den Berg, and JanC.T. Eijkel
}

\begin{abstract}
2D barrier materials such as graphene, boron nitride and molybdenum disulfide hold great promise for important applications such as DNA sequencing, desalination, and biomolecular sensing. The 2D materials commonly span pores through an insulating membrane and electrical fields are applied to drive cross-barrier transport of charged solvated species. While the low voltage transmembrane transport is well understood and controllable, high voltage phenomena are uncontrolled and result in the apparent breakdown of the 2D material's critical insulating properties. Here we use suspended graphene over a $50 \mathrm{~nm}$ silicon nitride nanopore as a model system and show that delamination of the 2D-material occurs at higher voltages and directly causes a number of the previously puzzling high voltage transport observations. We confirm the occurrence of delamination at $\sim 200 \mathrm{mV}$ and observe via AFM measurement a micron scale delaminated patch in a system using CVD graphene. Furthermore, we show that the conductivity of the same system is strongly correlated to the area of delamination via coincident current measurements and optical imaging of the delaminated area. Finally, we demonstrate that delamination alone can cause a dramatic breakdown of barrier function through observation of a reversible increase in conductance of samples prepared with pristine defect-free graphene. These findings should have a great impact on the design and interpretation of 2D-barrier material for both experiments and applications.
\end{abstract}



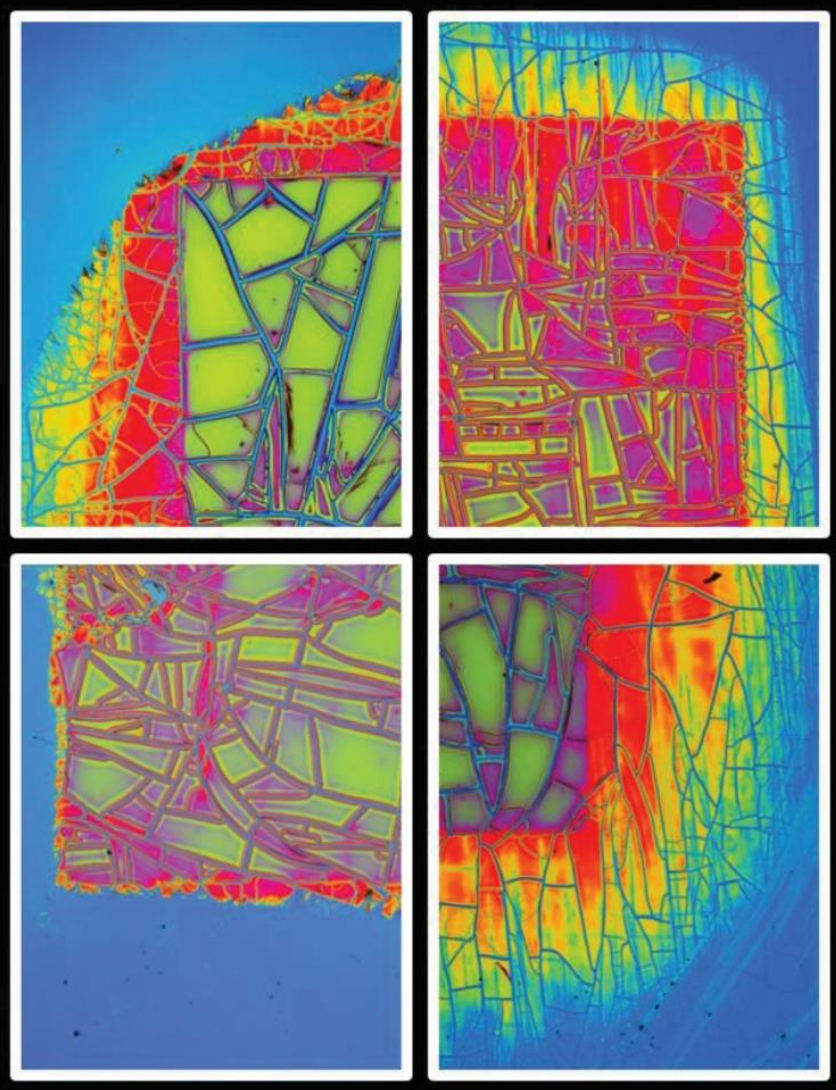


\section{Introduction}

2D materials have drawn much attention due to their exceptional mechanical, electrical and transport barrier properties.[1,2,3,4,5,6,7,8] Envisioned applications of such 2D material membranes abound, for electronic, mechanical or electromechanical devices, or as ultrathin barrier material for biomolecular sensing, desalination or DNA sequencing. $[9,10,11,12,13,14,15,16]$ When used as a barrier material in aqueous systems, their atomic thickness (sub-nm) provides a large advantage over other systems, as pores through the material exhibit exceptionally low transport resistance, while the materials themselves are generally impermeable. For such applications, native or manufactured pores of atomic $(\sim \mathrm{nm})$ scale in the barrier material are used. To create a functional barrier device, the 2D material is generally deposited on a carrier membrane made from silicon nitride (SiN) or silicon oxide $\left(\mathrm{SiO}_{2}\right)$ with larger diameter $(50 \mathrm{~nm}-5 \mu \mathrm{m})$ pores. In many cases, the device is then submerged in an aqueous solution and electrical fields are applied over this structure to drive ionic transport.

It has often been observed that applied voltages above a certain threshold (generally 200 to $500 \mathrm{mV}$ ) result in a breakdown of the resistance of the system, as indicated by a strongly non-linear and sometimes chaotic transmembrane current. $[8,9,17,18,19,20]$ This apparently consistent observation has been attributed to different phenomena in several studies, being labeled as non-understood [19], physical damage to the 2D material [9] delamination of the 2D material from the substrate [17], transient changes in the protonation of charged groups at the pore opening [18], or evidence for Coulomb blockade [20]. As it is often unclear whether this phenomenon permanently compromises the function of these systems, a proper understanding is of crucial importance.

Voltage-mediated delamination of the 2D material from the supporting membrane would explain the aberrantly high current at high voltage as it allows electrolyte to intrude under the $2 \mathrm{D}$ material. The inclusion of a layer of electrolyte between the 2D-material and membrane would provide an alternative conductive pathway through the system, and thus destroying the barrier function and causing the nonlinear current-voltage 
relation seen at higher voltages. Furthermore, this pathway can be intrinsically unstable, causing the chaotic system behavior that is often observed.

Voltage-mediated delamination has been observed with 2D materials in scenarios, such as the liquid-phase electrochemical exfoliation of bulk 2D materials and the electrochemical release of 2D materials from their substrate[21,22,23,24]. In these processes, a voltage applied to a bulk 2D material or the substrate on which a 2D material rests causes intercalation of ions which draw water with them, thus causing delamination. We hypothesized that a similar process is occurring for suspended 2D materials once the transmembrane voltage overcomes a threshold(Figure $6.1 \mathrm{c}$ and e). In this case, the electrolyte solution will begin to encroach into the interface between 2D material and substrate, if the resulting decrease in electrical capacitive energy on the 2D material outweighs the cost in energy for wetting the interface. This type of delamination would represent an inherent instability in any such suspended configuration in which a transmembrane voltage is applied.

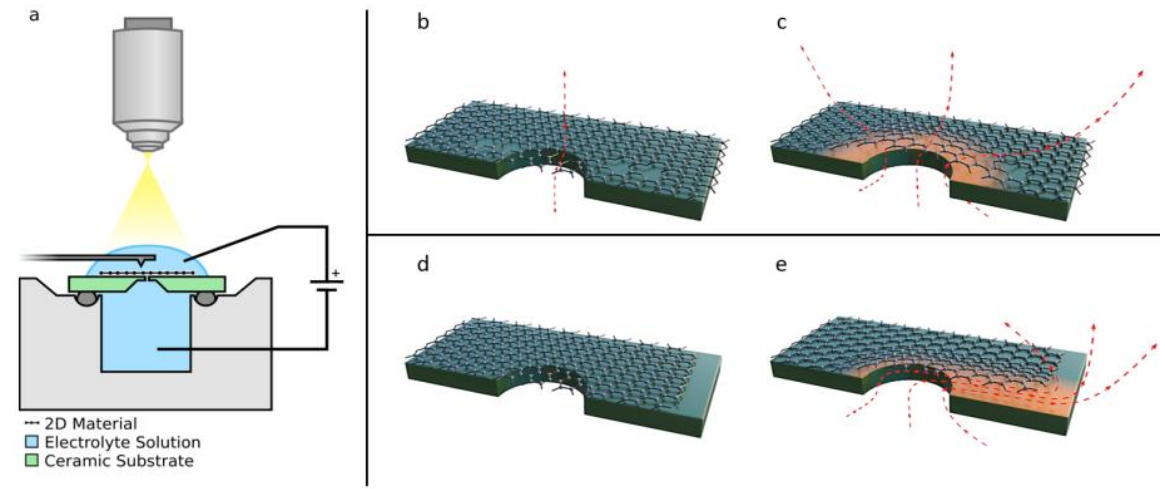

Figure 6.1: Schematic of a device in the test setup (a). The devices consists of a Si chip with a SiN carrier membrane (green) with graphene (CVD or pristine) overlaid (dotted line). The test setup allows application of voltage and amperometric measurement with coincident optical or AFM measurement. Panels b-e show schematically the system in all the states of the system examined in this paper: CVD graphene before delamination (b) CVD graphene after delamination(c), pristine graphene before delamination (d) and pristine graphene after delamination (e). Orange shaded areas denote the area of delamination and red lines indicate the path of current through the system. 
With an order of magnitude calculation, we can estimate the voltage regime in which delamination is expected. As the process should be spontaneous when the capacitive charging energy is larger than the interfacial energy change $\Delta \Gamma$, we can express the regime via the following inequality,

$6.1 \quad \frac{V^{2} C_{b}}{2}>\Delta \Gamma$.

Here the subscript $b$ refers to the $2 \mathrm{D}$ barrier material, $\mathrm{C}$ is the specific capacitance of the 2D-material and $\mathrm{V}$ is the voltage across the membrane.

Common 2D materials such as graphene, $\mathrm{MoS}_{2}$ and $\mathrm{BN}$ have comparable reported values for the properties appearing in equation 6.1. Specifically, dielectric constants $[25,26,27,28,43]$ thicknesses $[26,29,30,31]$ and adhesion energies to both water and ceramic substrates $[32,33,34,35,36,37,38,39,40,41]$ lie within the same order of magnitude respectively. The most broadly and consistently reported parameters are found for graphene-based systems. Therefore, for the remainder of the theoretical and experimental work presented here, we will work with graphene on SiN systems as a proxy for suspended monolayers of 2D materials in general. While this is not a perfect proxy it should serve to indicate the general range of conditions under which delamination should occur for suspended 2D materials in general.

We will first calculate the expected voltage of delamination to determine if it falls within the typical -1 to $1 \mathrm{~V}$ window where a breakdown is observed. As the SiN surface has been strongly oxidized in our case (see methods), we use surface energies associated with $\mathrm{SiO}_{2}$. Reliable estimates for the hydroxylated $\mathrm{SiO}_{2}$-water interfacial energy $\left(129 \mathrm{~mJ} / \mathrm{m}^{2}\right)$ and for the graphene-water interface $\left(29 \mathrm{~mJ} / \mathrm{m}^{2}\right)$ are available.[36,37] Literature values for the interfacial energy of the $\mathrm{SiO} 2 /$ graphene system, on the other hand, vary widely ranging from 450 to $96 \mathrm{~mJ} / \mathrm{m}^{2}$, and are thought to depend on the roughness of the carrier membrane as well as the presence of intermediate water.[38,39,40,41]. We will take the value of approximately $200 \mathrm{~mJ} / \mathrm{m}^{2}$ for the graphene- $\mathrm{SiO}_{2}$ energy in the presence of $\mathrm{H}_{2} \mathrm{O}$ [38]. By subtracting the $\mathrm{SiO}_{2}-$ water and graphene-water interfacial energies from the graphene- $\mathrm{SiO}_{2}$ interfacial energy an estimated interfacial energy change $\Delta \Gamma$ of $42 \mathrm{~mJ} / \mathrm{m}^{2}$ is 
obtained. The capacitor system that is charged consists of the anodic doublelayer capacitance (DLC), the graphene capacitance and the cathodic DLC in series. Taking respective values of $0.2,0.08$ and $0.08 \mathrm{~F} / \mathrm{m}^{2}[42,43]$, we estimate the series capacitance as $33 \mathrm{mF} / \mathrm{m}^{2}$. From these values for interfacial energy and capacitance, we expect a delamination voltage of about $1.6 \mathrm{~V}$. Though approximate, these calculations show delamination may occur near the voltages at which breakdown of these systems is commonly observed.

In the experimental part of this work, we consider the breakdown of barrier function for both pristine exfoliated graphene and for CVD graphene laden with nanopore defects. While both are expected to delaminate, the behavior of the system after delamination is expected to be slightly different and bears consideration. In both cases, once the voltage threshold for delamination is surpassed, the 2D material is expected to begin delaminating in the area directly around the pore through the substrate material. In the case of CVD graphene, (Figure $6.1 \mathrm{~b}$ and $\mathrm{c}$ ) as the delaminated patch grows the number of exposed defects increases and the observed electrical resistance of the system should decrease. As a result, the voltage under the 2D material is also expected to decrease with an increased delaminated area, eventually dropping below the voltage threshold for delamination. This would cause the delaminated area to stop growing, once it reaches a specific size. A simple resistive model of this effect can be found in Appendix 3. In the case of the pristine material, (Figure $6.1 \mathrm{~d}$ and e) there is no decrease in resistance until the delaminated area finds the edge of the $2 \mathrm{D}$ material. The delaminated patch is therefore expected to continue widening until it finds an edge. At this point, current can begin to flow and the voltage under the patch will begin to reduce. From this understanding, the voltage at the observed breakdown is expected to be the same for both CVD and pristine graphene, but the magnitude of current and area of delamination are expected to differ.

\section{Experimental and Discussion}

To experimentally probe the barrier function breakdown, we have fabricated suspended graphene (either CVD or pristine graphene) on SiN devices. Figure 6.1a schematically shows the device employed, where a graphene sheet is suspended over a SiN membrane with a $50 \mathrm{~nm} \varnothing$ nanopore, located between two reservoirs of aqueous salt solutions $(1 \mathrm{M} \mathrm{KCl})$, which are 
both equipped with a calomel electrode or silver/silver chloride electrode for voltage control.

First, we validate the occurrence of breakdown in our system via cyclic voltammetry and indeed observe a non-linear current-voltage relationship after a voltage threshold is crossed as previously observed in similar systems by: Jain et al. [18], Liu et al. [9], Feng et al. [10] and Cun et al. [8]. Results for CVD graphene in Figure 6.2 show the strongly non-linear increase of the current when the voltage exceeds a threshold value of about $0.25 \mathrm{~V}$. Subsequent scans ('cycle 1, 2, 3') show roughly similar responses. It is worth noting that this threshold voltage is lower than predicted, implying either a higher capacitance or a lower adhesion strength.

To determine if delamination is occurring concurrently, the graphene was visualized in-situ by in-liquid AFM at a series of fixed applied potentials in

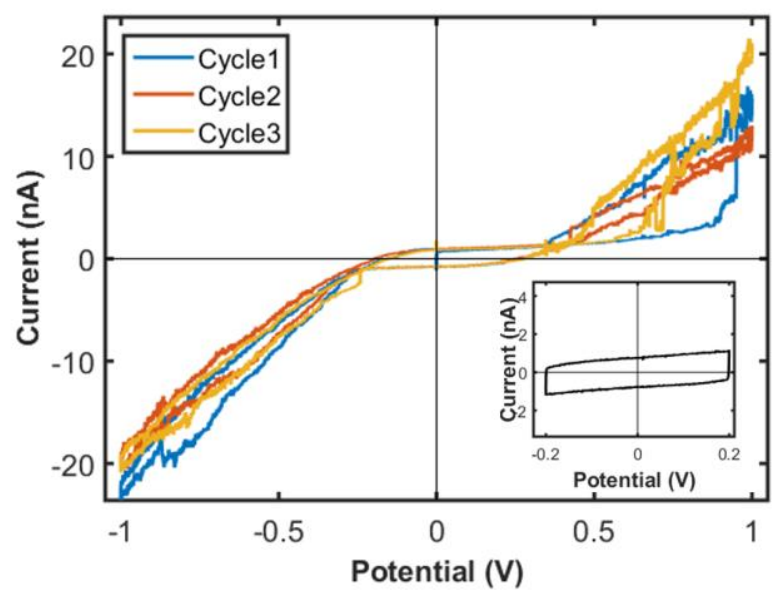

Figure 6.2: Sample cyclic voltammogram from the CVD graphene on SiN system. The dramatic increase in current at about $\pm 0.25 \mathrm{~V}$ constitutes the discussed breakdown of barrier function. Note that the current not only increases more steeply at higher voltages but also becomes somewhat chaotic, sometimes changing dramaticly for a small change in voltage. The inset shows the often reported current response for a low voltage sweep which shows no breakdown. It is worth noting that cyclic voltammetry results in a non-zero capacitive charging current at zero volts compared to the sequential fixed voltage measurements often used in the literature. This current is directly proportional to the scan rate and goes to zero for fixed voltage application. 
a similar range to that used during the cyclic voltammetry (Figure 6.3). A raised patch of graphene above the SiN pore can clearly be seen to appear and disappear with voltage, while the surrounding pre-existing wrinkles in the CVD graphene layer remain relatively unchanged. This clearly indicates that the application of voltage is causing the graphene to lift from the surface.

We also note from the AFM measurements that the delamination of the CVD graphene remains localized in our system. As mentioned before, we expect this because, as delamination progresses, the current through the system may pass through an increasing number of nanopore defects reducing the voltage under the graphene. With this understanding of the system, it becomes clear that the measured system conductance for CVD graphene is
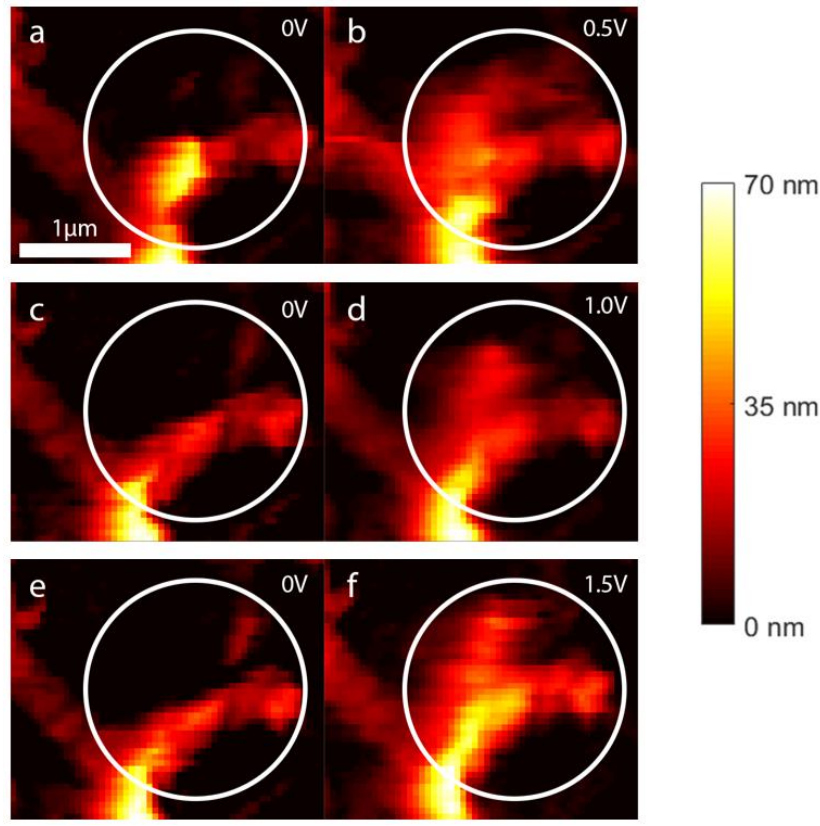

Figure 6.3: Sequential AFM measurements of changes in CVD graphene surface profile during various applied voltages. Voltages of $0.5(b), 1.0(d)$, and $1.5(f), V$ were used. After application of a given voltage a subsequent scan is performed at OV $(a, c, e)$ shown in the left column. A raised patch can be seen to appear and disappear with the application and removal of voltage implying that delamination is occurring. White circle indicates the area in which the SiN nanopore lies as validated by SEM imaging (Figure A3.7). Note that the elevated features during application of zero volts are preexisting wrinkle in the graphene. 
expected to be highly correlated with the observed delaminated area. To expand upon this, some basic modeling of the expected conductance and correlation to the delaminated area is included in Appendix 3.

We empirically determine the correlation between delaminated area and system conductance via coincident optical reflection microscopy and cyclic voltammetry. The delaminated area was calculated from the optical data and Figures $6.4 \mathrm{e}$ and $6.4 \mathrm{j}$ show the measured area plotted against the conductance. A strong correlation is observed in both cases, providing strong evidence that delamination is contributing to the observed nonlinear conductance.

As also observed by the AFM measurements, the local delamination in our system is strongly influenced by the local graphene topology, such as wrinkles, which may account for the heterogeneity within cycles and between different systems. The continuous and unpredictable formation of unstable, but sometimes permanent pathways can explain both the chaotic behavior and the permanent delamination observed. The discrete nature of defects in the CVD graphene may also contribute to the chaotic and step-like changes in current. This idea is further expanded upon in Appendix 3.

While the above explains why an increased delaminated area is often concurrent with an increased conductivity, it does not prove conclusively that delamination on its own can cause the observed breakdown of barrier function. Indeed we sometimes observed an elevated conductance without a direct increase in the delaminated area. Likewise, an increase in area is not always immediately followed by an elevated conductance (for example: Figure $6.4 \mathrm{i}, 500-600$ seconds). Other possible phenomena such as nonlinear transport through defects in the graphene or physical damage to the graphene may therefore also be at play. $[9,18,19,20]$

To test if delamination causes a breakdown in the absence of other possible causes, devices were prepared with defect-free exfoliated graphene and tested similarly with cyclic voltammetry (Figure 6.5). In this case, there are no defects so any observed breakdown in barrier function necessarily comes from either the delamination of the graphene or the permanent physical degradation of the graphene layer. 


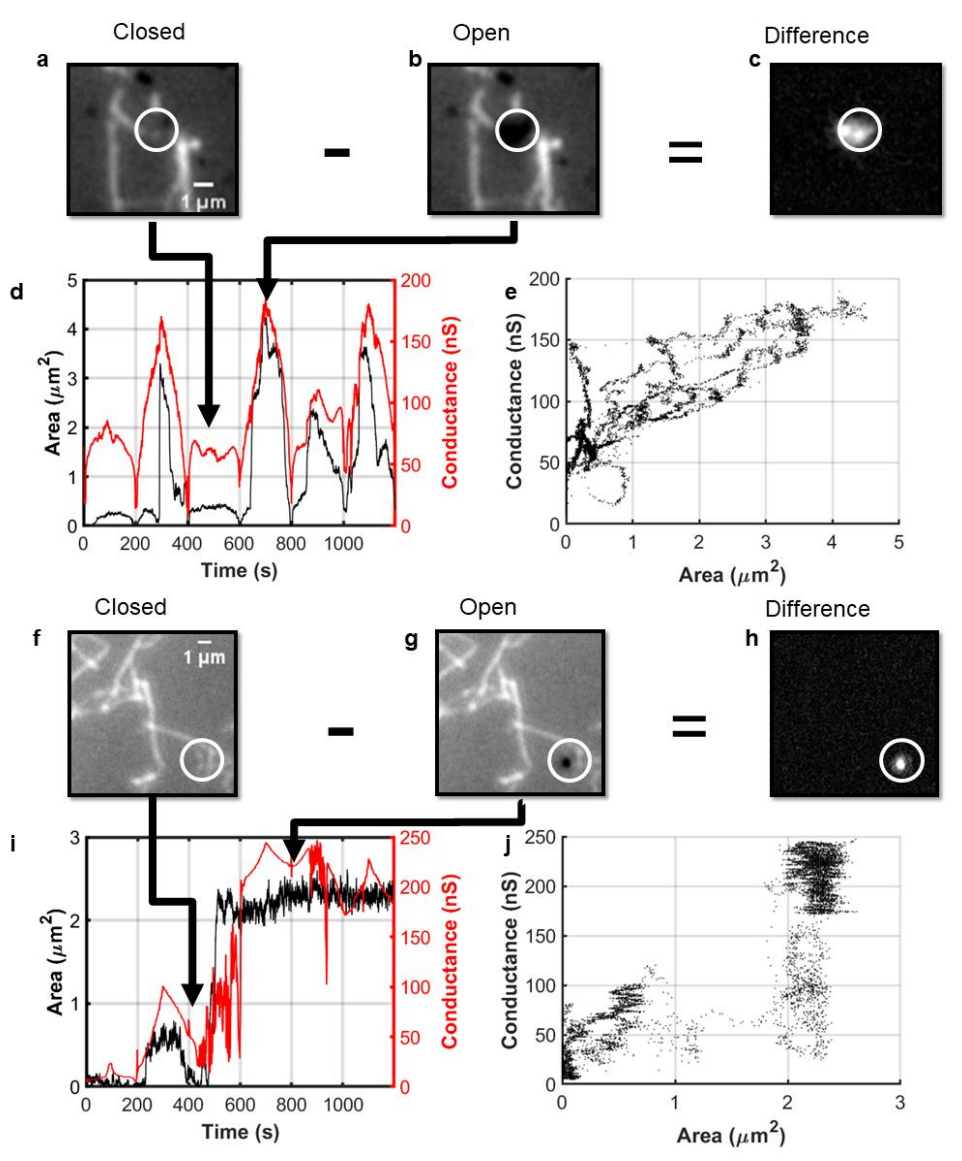

Figure 6.4: Optically determined area of delamination and coincident conductance of CVD graphene for two devices (a-e and $\mathrm{f}-\mathrm{j}$ ) plotted both against time $(d, i)$ and against each other $(e, j)$. The strong correlation shows that delamination often coincides with barrier breakdown and likely contributes to it. Sample optical data from region of delamination is also shown (a-c, $f-h)$ and differential images demonstrate that the effect is local as expected $(c, h)$. White circle indicates the area in which the SiN nanopore lies as validated by SEM imaging (Figure A3.7). The appearance of a dark spot indicates the formation of a blister of liquid under the graphene. Area of delamination is approximated by the number of pixels above a threshold value after normalization and background subtraction. Top data set shows a device where the graphene appears to delaminate at high voltage and relaminates at low voltage. Bottom dataset shows a device where the graphene remains delaminated after the initial delamination event and the conductance similarly remains high. Note that the white lines appearing on the optical images are preexisting wrinkles in the graphene. 
In these experiments, we indeed see a significant break down in barrier function at sufficiently high applied voltages as well as a total recovery of barrier function after returning to lower voltages. While physical degradation of the graphene may seem to be a likely explanation for the initial breakdown, it would result in a permanently increased conductance of the system, which was not observed. However, in the case of delaminationmediated breakdown, the increased conductance must be caused by the reversible opening of a conductive path under the graphene sheet from the ceramic pore to the edge of the graphene flake (Figure 6.1e). We note that the resulting path must be much longer (Figure A3.6) than the path under the graphene observed in the case of CVD graphene and thus the increase in conductivity is expected to be less dramatic.(See Appendix 3 for expanded explanation) Removal of voltage will allow this path to collapse and cause the conductance to recover to its initial level, matching our observations. Therefore we take the reversible breakdown of barrier function in the case of pristine graphene as confirmation that delamination can be solely responsible for the breakdown in suspended 2D material systems.

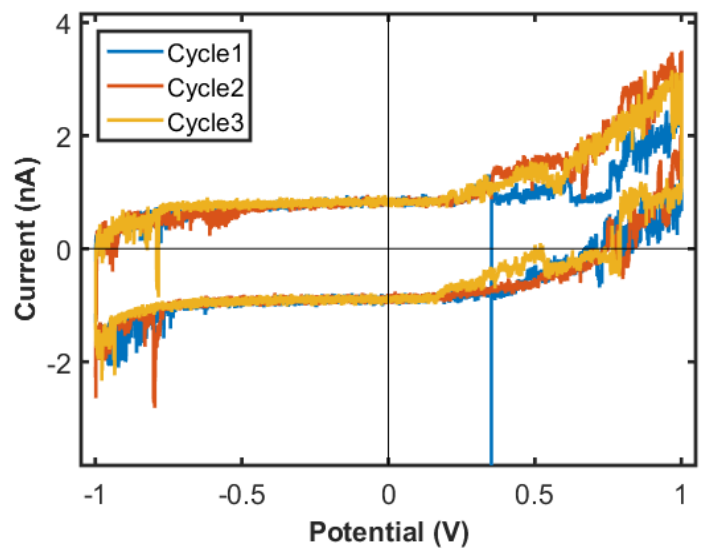

Figure 6.5: Three cyclic voltammograms of the pristine graphene-on-SiN system. Breakdown is again indicated by a nonlinear increase in the current at about 0.25 volts and again the current not only increases more steeply but also becomes chaotic. In contrast to the CVD graphene there are no nanopore defects to contribute to the current and the reversibility of the breakdown implies that there is no physical damage to the graphene itself. Therefore delamination remains the single explanation for the observed breakdown under these conditions. 


\section{Summary and Conclusion}

We have investigated voltage-mediated delamination of suspended 2D materials via a graphene on SiN system and observed the effect of delamination on the breakdown of barrier function. For both exfoliated and CVD graphene, we observe breakdown of the system reliably at $\sim 200 \mathrm{mV}$. Via in-liquid AFM, we confirmed that a micron-scale raised blister in the CVD graphene does indeed occur above the $50 \mathrm{~nm}$ SiN pore at above the breakdown voltage. Next, we found that the degree of delamination of the CVD graphene is strongly correlated with the conductance of the system during breakdown, which indicate $s$ with a strong likelihood that delamination contributes to the breakdown. We then demonstrated that delamination can be the sole cause of breakdown by repeatedly inducing and reversing breakdown of a system made with mechanically exfoliated pristine graphene. These results show that voltage-mediated delamination needs to be accounted for when designing systems that utilize suspended 2D materials. If the barrier function of the $2 \mathrm{D}$ material is critical, steps need to be taken to enhance adhesion of the 2D material, especially when higher transmembrane voltages are needed.

\section{Conflicts of Interest}

There are no conflicts of interest to declare.

\section{Acknowledgements:}

We would like to thank Vasilis Papadimiriou for much fruitful discussion and help with student supervision. Henk van Wolferen also was of great assistance for much of the focused ion beam milling. This work was supported in part by the Netherlands Center for Multiscale Catalytic Energy Conversion (MCEC), an NWO Gravitation program funded by the Ministry of Education, Culture, and Science of the government of the Netherlands. B. Radha acknowledges Royal society Fellowship R123098 and RGF\EA \181000 for contribution to this work. E. Grady acknowledges the financial support of the Dutch Technology Foundation STW (project number 140930), which is part of the Netherlands Organization for Scientific Research (NOW). A. Keerthi thanks Ramsey Memorial Fellowships Trust for fellowship. 


\section{Materials and Methods}

\section{Silicon nitride membrane fabrication}

A $50 \mathrm{~nm}$ thick membrane of stoichiometric silicon nitride was used as a membrane for the support of the graphene sheet. Conventional lithography and dry etching $\left(\mathrm{CHF}_{3}\right.$ and $\left.\mathrm{O}_{2}\right)$ were used to pattern and etch a window in the back-side silicon nitride. Subsequently, a silicon pit was etched by $\mathrm{KOH}$ wet etching $\left(25 \%{ }_{w}\right.$ at $75^{\circ} \mathrm{C}$ for about 9 hours). Leaving a pit that tapers from $\sim 1$ $\mathrm{mm}$ to $\sim 80 \mu \mathrm{m}$. The final silicon nitride membrane thickness was approximately $41 \mathrm{~nm}$ as a result of this $\mathrm{KOH}$ wet etch. $50 \mathrm{~nm}$ of chromium was deposited on the front silicon nitride by e-beam evaporation to prevent the charging of silicon nitride during the nanopore drilling. The nanopore was drilled via Focused Ion Beam milling (FEI Nova600 Dual-beam FIB/SEM) resulting in pores of about $50 \mathrm{~nm}$ in diameter. After the FIB process, the wafer was diced into $16 \times 16 \mathrm{~mm}^{2}$ chips. Next, a standard $\mathrm{Cr}$ wet etch solution (ceric(IV) ammonium nitrate-based etchant) was used to strip the chromium, where after the silicon nitride membranes were thoroughly cleaned using $\mathrm{RCA} 2\left(\mathrm{HCl} / \mathrm{H}_{2} \mathrm{O}_{2} / \mathrm{H}_{2} \mathrm{O}\right.$ 1:1:5 at $\left.80^{\circ} \mathrm{C}\right)$ and piranha $\left(\mathrm{H}_{2} \mathrm{SO}_{4} / \mathrm{H}_{2} \mathrm{O}_{2} 3: 1\right.$ at $\left.95^{\circ} \mathrm{C}\right)$. Afterwards, a thermal oxidation process ( 45 min wet oxidation at $900{ }^{\circ} \mathrm{C}$ ) was performed to prevent a current leakage path through the Si substrate.

\section{Graphene growth and transfer protocol}

Monolayer graphene was grown in collaboration at the Plasma \& Materials Processing (PMP) department of Eindhoven University of Technology. The graphene was synthesized on a copper foil (99.8\% Alfa Aesar no. 13382) by means of low-pressure chemical vapor deposition (LPCVD) in a tube furnace. Prior to the synthesis, the copper sheets were cleaned with acetone for $30 \mathrm{~min}$, methanol for $1 \mathrm{~min}$ and finally $1 \mathrm{M}$ nitric acid for $30 \mathrm{sec}$.

The growth process was run at $1050{ }^{\circ} \mathrm{C}$. First, the quartz tube was purged with argon gas. Thereafter, the tube was pumped down to $5 \times 10^{-3}$ mbar. Next, the copper was annealed for $45 \mathrm{~min}$ with a hydrogen flow of 10 sccm and an argon flow of 500 sccm. Lastly, the graphene was grown for 20 min with an additional methane flow of $100 \mathrm{sccm}$, a reduced hydrogen flow of $6 \mathrm{sccm}$ and an argon flow of $500 \mathrm{sccm}$. The quality of the monolayer graphene was verified by Raman spectroscopy.[44,45] 
The synthesized monolayer graphene was transferred to a silicon nitride substrate using a wet transfer protocol with poly(methyl methacrylate) (PMMA) as the support polymer.[46] A PMMA layer of approximately $100 \mathrm{~nm}$ was spin coated on top of the graphene, after which a copper etch using a $40 \mathrm{mM}$ concentration of ammonium persulfate was performed. Before transfer, the silicon nitride membranes were also thoroughly cleaned using $\mathrm{HNO}_{3}(69 \%), \mathrm{RCA} 2\left(\mathrm{HCl}: \mathrm{H}_{2} \mathrm{O}_{2}: \mathrm{H}_{2} \mathrm{O}, 1: 1: 5\right.$ at $\left.80^{\circ} \mathrm{C}\right)$, piranha all at $80^{\circ} \mathrm{C}$ for at least 15 min each with $3 x$ rinsing with fresh DI water between each step.

Exfoliated graphene was provided by the condensed matter physics group of the University of Manchester. A monolayer of graphene (approximately $50 \mu \mathrm{m} \times 100 \mu \mathrm{m}$ ) was prepared by mechanical exfoliation [47] from natural graphite (NGS naturgraphit $\mathrm{GmBH}$, Germany) using scotch tape on a $\mathrm{Si}$ wafer with $290 \mathrm{~nm}$ of $\mathrm{SiO}_{2}$. The prepared graphene monolayer was transferred onto the free-standing silicon nitride membrane using a PMMA based wet transfer method.[48] To ensure that the flake would not totally delaminate when voltage was later applied, the exfoliated flake was placed carefully such that the edge of the flake was near (within $\sim 10 \mu \mathrm{m}$ ) the nanopore in the SiN substrate. (Figure A3.7) After the transfer, the polymer residues were thoroughly washed with acetone, isopropyl alcohol and finally rinsed with hexane.

\section{Current measurements}

The devices were first introduced into a two-reservoir device holder and the reservoirs were filled with $1 \mathrm{M} \mathrm{KCl}(99 \%$ potassium chloride of Sigma Aldrich dissolved in Milli-Q water).

The current measurements were performed using either a Bio-Logic VSP potentiostat or a Bio-Logic SP300 potentiostat in combination with either saturated calomel electrodes (Radiometer Analytical XR110) or silver/silver chloride electrodes. The silver/silver chloride electrodes were synthesized by electro-deposition and were only used in combination with the Bio-Logic VSP potentiostat for optical reflection microscopy and AFM measurements. Due to space constraints, all other measurements were done with calomel electrodes. The current measurements on the pristine graphene were performed with the Bio Logic SP300. CV measurements done in conjunction 
with optical measurements were performed with a scan rate of $25 \mathrm{mV} / \mathrm{s}$ while all others measurements were performed at $10 \mathrm{mV} / \mathrm{s}$. All electrochemical measurements were performed using EC-lab software.

\section{Optical measurements}

The setup in figure A3.1 was used for the simultaneous recording of optical reflection measurements and current measurements while applying a voltage sweep. The optical setup consists of a Leica DM2500 MH optical microscope with a $63 x$ objective. The images were recorded using a PCO Pixelfly 14 bit digital CCD camera system. The microscope was operated in reflection mode.

Data processing of the images is done using MATLAB R2017b. Drift was compensated for using a geometric translation algorithm. Furthermore, the image intensity was normalized and subsequently a background subtraction was performed. Finally, the size of the delaminated graphene was determined by counting all the pixels above a certain threshold value.

\section{AFM measurements}

The photo in figure A3.S shows the simultaneous recording of the inliquid AFM measurements while applying constant voltage. The AFM measurements were performed with an Agilent 5100 AFM, operating in tapping mode. A NSC35a AFM tip from MikroMasch with a typical resonance frequency of $205 \mathrm{kHz}$ and a force constant of $8.9 \mathrm{~N} / \mathrm{m}$ was used. Images were collected with a resolution of 512 measurement points per line at $2 \mathrm{~Hz}$ over an area of $20 \times 20 \mu \mathrm{m}^{2}$. The AFM data was processed using Gwyddion v2.40. Mean plane subtraction and polynomial background removal were used to level the data. Further processing was done in MATLAB R2017b to normalize and remove the background. Multiple scans at $0.5,1$ and $1.5 \mathrm{~V}$ were averaged in the shown data.

\section{Growth of silver/silver chloride electrodes}

Silver/silver chloride electrodes were synthesized using electrodeposition on a silver wire with a diameter of $0.5 \mathrm{~mm}$. Before electrodeposition, the wires were cleaned with fine sandpaper and submerged in

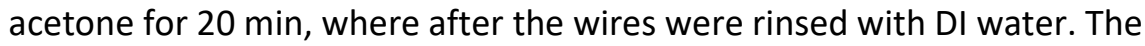


formed silver chloride layer was grown for $1 \mathrm{~h}$ at $21.15 \mu \mathrm{A}$ in a solution of 0.1 $\mathrm{M} \mathrm{HCl}$ and $1 \mathrm{M} \mathrm{KCl}$. 


\section{Reference}

1 J.M. Hamm and O. Hess, Two Two-Dimensional Materials Are Better than One, Science 340, 2013, 1298-1299

${ }^{2}$ K. S. Novoselov, D. Jiang, F. Schedin, T. J. Booth, V. V. Khotkevich, S. V. Morozov, and A. K. Geim, Two-dimensional atomic crystals, Proc. Natl Acad. Sci. USA 102, 2005, 10451-10453

${ }^{3}$ K. S. Novoselov, A.K. Geim, S. V. Morozov, D. Jiang, Y. Zhang, S.V. Dubonos, I.V. Grigorieva, A.A. Firsov, Electric field effect in atomically thin carbon films, Science 306, 2004, 666-669

${ }^{4}$ S.Z. Butler et al., Progress, Challenges, and Opportunities in Two-Dimensional Materials Beyond Graphene, ACS Nano 7, 2013, 2898-2926

${ }^{5}$ S.Guo and S.Dong, Graphene nanosheet: synthesis, molecular engineering, thin film, hybrids, and energy and analytical applications, Chem. Soc. Rev. 40, 2011, 2644-2672

${ }^{6}$ G. Fiori, F. Bonaccorso, G. lannaccone, T. Palacios, D. Neumaier, A. Seabaugh, S.K. Banerjee and L. Colombo, Electronics based on two-dimensional materials, Nature Nanotech. 9, 2014, 768-779

${ }^{7}$ S.Liu et al., Boron Nitride Nanopores: Highly Sensitive DNA Single-Molecule Detectors, Adv. Mater. 25, 2013, 4549-4554

${ }^{8} \mathrm{H}$. Cun et al., Centimeter-Sized Single-Orientation Monolayer Hexagonal Boron Nitride With or Without Nanovoids, Nano Lett. 18, 2018, 1205-1212

${ }^{9}$ K.Liu, J. Feng, A. Kis and A. Radenovic, Atomically Thin Molybdenum Disulfide Nanopores with High

Sensitivity for DNA Translocation, ACS Nano 8, 2014, 2504-2510

$10 \mathrm{~J}$. Feng, K. Liu, R.D. Bulushev, S. Khlybov, D. Dumcenco, A. Kis and A. Radenovic, Identification of single nucleotides in MoS2 nanopores, Nature Nanotech., 10, 2015, 1070-1076

${ }^{11}$ S. Garaj, W. Hubbard, A. Reina, J. Kong, D. Branton11 and J.A. Golovchenko, Graphene as a subnanometre trans-electrode membrane. Nature 467, 2010, 190193.

${ }^{12}$ C.A. Merchant, K. Healy, M. Wanunu, V. Ray, N. Peterman, J. Bartel, M.D. Fischbein, K. Venta, Z. Luo, A.T.C. Johnson, and M. Drndic, DNA Translocation through Graphene Nanopores, Nano Lett. 10, 2010, 2915-2921

${ }^{13}$ G.F. Schneider, S.W. Kowalczyk, V.E. Calado, G. Pandraud, H.W. Zandbergen, L.M.K. Vandersypen, and C. er, DNA Translocation through Graphene Nanopores, Nano Lett. 10, 2010, 3163-3167

${ }^{14} \mathrm{~S}$. Heerman, and C. Dekkar. Graphene nanodevices for DNA sequencing. Nature Nanotech. 11, 2016, 127-136.

${ }^{15}$ B. Venkatesan, R. Bashir. Nanopore Sensors for Nucleic acid Analysis. Nature Nanotech. 6, 2011, 115-624.

${ }^{16}$ Y Wu, R. Tilley, J. Gooding. Challenges and Solutions in Developing Ultrasensitive Biosensors, Journal of the American Chemical Society, 141, 2019, 1162-1170. 
${ }^{17}$ A.Esfandiar, B. Radha, F.C. Wang, Q. Yang, S. Hu, S. Garaj, R.R. Nair, A.K. Geim, K. Goopinadhan, Size effect in ion transport through angstrom-scale slits, Science, 358, 2017, 511-513.

${ }^{18}$ T. Jain, B.C. Rasera, R.J.S. Guerrero, M.S.H. Boutilier, S.C. O'Hern, J.C. Idrobo and Rohit Karnik, Heterogeneous sub-continuum ionic transport in statistically isolated graphene nanopores, Nature Nanotech. 10, 2015, 1053

${ }^{19}$ S.P. Surwade, S.N. Smirnov, I.V. Vlassiouk, R.R. Unocic, G.M. Veith, S. Dai and S.M. Mahurin, Water desalination using nanoporous single-layer graphene, Nature Nanotech., 2015, 459-464

${ }^{20}$ J. Feng, K. Liu, M. Graf, D. Dumcenco, A. Kis, M. Di Ventra and A. Radenovic, Observation of ionic Coulomb blockade in nanopores, Nature Materials, 15, 2016, 850-856

${ }^{21} \mathrm{~J}$. Zhang, E.Wang, STM investigation of HOPG superperiodic features caused by electrochemical pretreatment, J. Electroanal. Chem. 399, 1995, 83-89

${ }^{22}$ D. Alliata, P. Häring, O. Haas, R. Kötz, H. Siegenthaler, Anion intercalation into highly oriented pyrolytic graphite studied by electrochemical atomic force microscopy, Electrochem. Comm. 1, 1999, 5-9

${ }^{23}$ K. Verguts, J. Coroa, C. Huyghebaert, S. De Gendt, S. Brems. Graphene delamination using 'electrochemical Methods': an ion intercalation effect.

Nanoscale. 10, 2018, 5515-5521.

${ }^{24}$ Y. Wang, Y. Zheng, X. Xu, E. Dubuisson, Q. Bao, J. Lu, K. Loh. Electrochemical Delamination of CVD Grown Graphene Film: Towards Recyclable use of Copper Catalyst. ACS Nano. 5, 2011, 9927-9933.

${ }^{25}$ C. Dean, A. Young I.Meric, C. lee, L. Wang, S. Sorgenfrei K. Watanbe, T. Taniguci, P. Kim, K. Shepard, J.Hone, Boron Nitride Substrates for High-Quality Graphene Electronics. Nature Nanotech, 5, 2010, 722-726.

${ }^{26}$ X, Chen, Z. Wu, S. Xu, L. Wang, R. Huang, Y. Han, W. Ye, W. Xiong, T. Han, G. Long, Y. Wang, Y. He, Y. Cai, P. Sheng, N. Wang. Probing electron states and electroninsulator transition mechanisms in molybdenum-disulfide vertical heterostructures. Nature Communications. 6, 2015, 6088.

${ }^{27}$ T. Cheiwchanchamnangij and W. Lambrecht. Quasiparticle band structure calculation of monolayer bilayer and bunk MoS2. Physical Review B. 85, 205302, 2012.

${ }^{28}$ A. Molina-Sanchez, and L. Wirtz. Phonons in single layer and few layer $\mathrm{MoS}_{2}$ and WS. Physical Review B. 84 155413, 2011.

${ }^{29}$ S. Liu, B. Lu, Q. Zhao, J. Li, T. Gao, Y. Chen, Y. Zhang, Z. Liu, Z. Fan, F. Yang, L. You, and D. Yu. Boron Nitride Nanopores: Highly Sensitive DNA Single-Molecule Detectors. Advanced Materials. 25, 2013, 4549-4554.

${ }^{30}$ M. Joo, B. Moon, H. Ji, G. Han, H. Kim. G. Lee, S. Lim, D. Suh, and Y. Lee. Electron Excess Doping and Effective schottkey barrier reduction on the $\mathrm{MoS}_{2} / \mathrm{h}-\mathrm{BN}$ heterostructure. Nano letters. 16, 2016, 6383-6389.

${ }^{31}$ Z. Ni, H. Wang, J. Kasim, H. Fan, T. Yu, Y. Wu, Y. Fang, and Z. Shen. Graphene Thickness Determination Using Reflection and Contrast Spectroscopy. Nano Letters. 7, 2007, 2758-2763. 
${ }^{32}$ C. Lee, Q. Li, W. Kalb, X. Liu, H. Berger, R. Carpick, and J. Hone. Frictional Characteristics of Atomically Thin Sheets. Science. 2, 2010, 76-80.

${ }^{33}$ K. Yum, and M. Yu. Measurement opf Wetting Properties of Individual Boron Nitride Nanotubes with the Wilhelmy Method Using a Nanotube-Based Force Sensor. Nano Letters. 6, 2016, 329-333.

${ }^{34}$ S. Deng, E. Gao, Z. Xu, and V. Berry. Adhesion Energy of $\mathrm{Mo}_{2} \mathrm{~S}$ Thin Films on Silicon Based Substrates Determined via the Attributes of a Single $\mathrm{MoS}_{2}$ Wrinkle. Applied Materials and Interfaces. 9, 2017, 7812-7818.

${ }^{35}$ A. Kozbial, X. Gong, H. Liu, and L. Li. Understanding the intrinsic Water Wettability of Molybdenum Disulfide (MoS 2 ). Langmuir. 31, 2015, 8429-8435.

${ }^{36} \mathrm{~S}$. Brunauer, D. Kantro, and C. Weise. The surface energies of amorphous silica and hydrous amorphous silica. Canadian J. Chem., 34, 1956, 1483-1496

${ }^{37}$ S. Wang, Y. Zhang, N. Abidi, and L. Cabrales. Wettability and surface free energy of graphene films, Langmuir, 25, 2009, 11078-11081

${ }^{38}$ W. Gao, P. Xiao, G. Henkelman, K. M. Liechti, and R. Huang. Interfacial adhesion between graphene and silicon dioxide by density functional theory with van der Waals corrections. Journal of Physics D: Applied Physics, 47, 2014, 255301

${ }^{39}$ E. Paek and G.S. Hwang. A Computational Analysis of Graphene Adhesion on Amorphous Silica, Journal of Applied Physics ,113, 2013, 164901

${ }^{40}$ K. Yue, W. Gao, R. Huang, K. Liechti. Analytical Methods for the Mechanics of Graphene Bubbles, Journal of Applied Physics. 112, 2012, 083512.

${ }^{41}$ N. Boddeti, X. Liu, R. Long, J. Xiao, J. Bunch, M. Dunn. Graphene Blisters with Switchable Shapes Controlled By Pressure and Adhesion, Nano Letters. 2103, 13, 6216-6221.

${ }^{42}$ G. Jiang, C. Cheng, D. Li and J.Z. Liu, Molecular dynamics simulations of the electric double layer capacitance of graphene electrodes in mono-valent aqueous

electrolytes, Nano Res. 9, 2016, 174-186

43 J. Xia, F. Chen, J. Li, and N. Tao, Measurement of the quantum capacitance of graphene, Nature Nanotech., 4, 2009, 505-509

${ }^{44}$ Ferrari, Andrea C., et al. Raman spectrum of graphene and graphene layers, Physical review letters 97, 2006, 187401.

${ }^{45}$ Malard, L. M., et al. Raman spectroscopy in graphene, Physics Reports 473, 2009, 51-87.

${ }^{46}$ van den Beld, Wesley Theodorus Eduardus, Graphene and permalloy integration in functional fluidic and solid-state devices, Thesis from: University of Twente, 2016.

${ }^{47}$ Geim, A. K. \& Grigorieva, I. V., Van der Waals heterostructures, Nature, 499, 2013 419-425.

${ }^{48}$ Novoselov, K. S.; Mishchenko, A.; Carvalho, A. \& Castro Neto, A. H., 2D materials and van der Waals heterostructures, Science 353, 2016, 9439. 


\section{Summary and Outlook}

In this section we will briefly summarize the work from each chapter and give an indication as to the next steps or broader impact of the work. In the first three chapters, particularly between chapters two and three, points from the outlook of one chapter directly inform parts of the motivation for the next. The final three chapters explore more general and varied problems with their outlooks similarly more outwards facing.

A summary in Dutch follows the one in English. 


\section{Chapter 1}

In Chapter 1 we report on the design and testing of a new technique for defining the shape of gel structures for 3D cell culture in microfluidic devices. Our technique can reproduce the most commonly used gel structures, but differs from existing techniques in that it uses laminar flow patterning to define the gel geometry instead of confining structures like pillars or phase guides. This both allows rapid prototyping of the gel geometry and reduces contact between cells and the unnaturally stiff material that makes up common devices.

We show that the technique can create a wide variety of geometries, both common in the literature and novel. The width of the gel is easily controlled in our technique by adjusting flow parameters during patterning. Once our gels are patterned and cured, cells seeded in these devices grow as expected. Finally, we show that devices can withstand trans-gel pressures far in excess of what is needed to reproduce physiological conditions making them useful to study the effect of these flows on cells cultured within. Taken together, the features provided by this technique should make it a powerful tool for fast implementation and development of new 3D cell culture devices.

\section{Chapter 2}

In Chapter 2 we show that 2D and 3D laminar flow patterning through a cured hydrogel is not only possible, but simple and precise. The flat plug flow profile common to flows through hydrogels actually makes the calculations required to fine tune and control the technique much simpler than those needed in a channel without hydrogel. Through testing of our devices we demonstrate excellent spatial control of the generated concentration profile via manipulation of the inlet pressures. Furthermore, we show that the technique can be used in a truly 3D capacity by 3D printing and testing a version of our device that allows for both horizontal and vertical control of the concentration profile as it flows downstream.

Although we only show the ability to manipulate concentration profiles in a gel in this chapter, we began work to demonstrate the potential of this technique to influence 3D cell cultures, which is reported in the next chapter. In addition to biological applications, our technique can serve as a general microfluidic tool to translate the types of surface patterns attainable with laminar flow patterning to 3D patterns in a hydrogel matrix. For example, our method could allow control over local stiffness of gels in 3D by manipulating the concentration profile of a cross-linker within the gel. 


\section{Chapter 3}

In Chapter 3 we use the technique developed in Chapter 2 to manipulate the concentration profile in the interstitium around a microvascular network. Our technique can recreate concentration profiles commonly used in in the organ-on-chip literature, but should allow simultaneous perfusion of the microvascular network. In the future, we plan to engineer fine-scale microvascular networks with this technique and show its efficacy for the modeling of relatively complex biological phenomena, like the effect on microvasculature of growth factor plumes.

The device design we present may also find wider application in the fields of organ-on-chip and tissue engineering. Our device is simple to use and similar to typical platforms used in 3D cell culture, but affords the user the additional ability to generate complex concentration profiles within the hydrogel. The ability to locally apply growth factors to 3D cell cultures with both spatial and temporal control is a step towards creating truly physiological conditions on chip and may be of assistance to a variety of 3D cell culture researches such as: the modeling of tumor-vessel interactions, differentiation of stem cells in 3D culture and the design of more complex and functional organoids.

\section{Chapter 4}

The technique presented in Chapter 4 allows relatively cheap and robust quantification of the diffusivity of proteins. The extensive internal quality controls detailed therein allow easy diagnosis of problems with implementation and ensure that the final results are of a high quality. To demonstrate the efficacy of this approach, we implemented a relatively unoptimized setup and measured the diffusivity of a panel of common cytokines. After applying quality controls, our setup yields values of diffusivity that fall within the literature expected range, demonstrating the robustness and accuracy of the technique.

Concentration landscapes of growth factors are becoming more and more complex in fields that apply 3D cell cultures. However, the diffusivities of the growth factors that make up these landscapes are still often only measured as needed due to the massive number of potentially useful peptides. As such, there is an increasing need for techniques that can easily validate the transport properties of the used peptides. We propose that the technique presented in Chapter 5 fills this niche nicely as it is relatively cheap to implement when compared to existing techniques and uses materials and experimental conditions common to 3D cell culture research. 


\section{Chapter 5}

In Chapter 5 we have shown that the often cumbersome task of multiplexing microfluidic devices can be surmounted by including a multiplexer made from clamped gasket valves within a chip holder. With our chip holder design, we demonstrate control over the pressure in many independent outlet channels using only two input signals. We next plan to show that this chip holder interfaces easily with many different microfluidic devices.

The novel use of impregnated gaskets to make leak-free and biased valves dramatically eases circuit design constraints common to working with elastomeric valves. Explicitly, the ability to switch large pressures with smaller ones removes the need for precisely tuned resistor networks to supply ever decreasing pressure to the switched side of the valves. The number of valves used in a device can therefore be scaled up dramatically with little design overhead.

The simple multilayer design used here can facilitate the translation of many different kinds of digital electronic circuits into pneumatic devices. For example, digital to analogue controllers would be capable of turning a series of on or off pneumatic inputs into a variable pressure or variable flow outputs. Similarly, programmable state machines, as have been demonstrated in PDMS devices, could be integrated for in-chip holder process automation. Ultimately integration of multiplexing and various other functions into a single chip holder could virtually remove the need for microfluidic peripherals.

\section{Chapter 6}

In Chapter 6 we observed that delamination of a 2D material from its substrate can, on its own, cause the commonly observed voltage mediated breakdown of suspended 2D material systems. We confirmed this observation via a variety of measurements, particularly: cyclic voltammetry, in-liquid atomic force microscopy (AFM) and white light reflectance microscopy.

For both exfoliated and chemical vapor deposited (CVD) graphene suspended on a SiN membrane with a single nanopore, we observed the reliable breakdown of the suspended material system at $\sim \pm 200 \mathrm{mV}$. Via inliquid AFM, we confirmed that a micron-scale raised blister in the CVD graphene, indicating local delamination, does indeed appear above the SiN 
pore when the breakdown is observed. Next, we found via coincident optical and amperometric measurement that, when using CVD graphene, the area of the delaminated patch is strongly correlated with the conductance of the system during breakdown. We then demonstrated that delamination can be the sole cause of breakdown by repeatedly inducing and reversing breakdown of a system made on a sample of exfoliated graphene with no observed conductive defects.

These results show that voltage-mediated delamination can be a real problem and should be accounted for when designing systems that utilize supported 2D materials and require voltage application. In the particular case of suspended 2D materials, steps need to be taken to enhance adhesion to the substrate when higher transmembrane voltages need to be applied. 


\section{Hoofdstuk 1}

In hoofdstuk 1 rapporteren we over het ontwerp en het testen van een nieuwe techniek voor het definiëren van de vorm van gelstructuren voor 3D-celcultuur in microfluïdische chips. Onze techniek kan de meest gebruikte gelstructuren reproduceren, maar verschilt van bestaande technieken doordat het een patroon door middel van laminaire stroming gebruikt om de gelgeometrie te definiëren in plaats van beperkende structuren zoals pilaren of fasegeleiders. Dit maakt het mogelijk om zowel snelle prototyping van de gelgeometrie te doen en het vermindert contact tussen cellen en het onnatuurlijk stijve materiaal waaruit gewone chips bestaan.

We laten zien dat de techniek een grote verscheidenheid aan geometrieën kan creëren, zowel de gebruikelijke uit de literatuur als nieuwe geometrieën. De breedte van de gel kan eenvoudig worden aangepast in onze techniek door de stroomparameters aan te passen tijdens het vormen van patronen. Zodra onze gels van een patroon zijn voorzien en zijn uitgehard, groeien cellen die in deze chips zijn gezaaid zoals verwacht. Ten slotte laten we zien dat de chips trans-geldrukken kunnen weerstaan die veel hoger zijn dan nodig is om fysiologische stroomsnelheden te reproduceren, waardoor ze nuttig zijn om het effect van deze stromen op de gekweekte cellen te bestuderen. Alles bij elkaar genomen zouden de functies van deze techniek het een krachtig hulpmiddel moeten maken voor snelle implementatie en ontwikkeling van nieuwe 3D-celcultuurapparaten

\section{Hoofdstuk 2}

In hoofdstuk 2 laten we zien dat 2D en 3D laminaire stromingspatronen door een uitgeharde hydrogel niet alleen mogelijk, maar ook eenvoudig en nauwkeurig zijn. Het vlakke plugstroomprofiel dat gebruikelijk is voor stromingen door hydrogels maakt de berekeningen die nodig zijn om de techniek fijn af te stellen en te besturen veel eenvoudiger dan die nodig zijn in een kanaal zonder hydrogel. Door het testen van onze chips tonen we een uitstekende ruimtelijke controle van het gegenereerde concentratieprofiel via manipulatie van de ingangsdrukken. Verder laten we zien dat de techniek in 3D kan worden gebruikt door het 3D-printen en testen van een versie van onze chip die zowel horizontale als verticale controle van het concentratieprofiel mogelijk.

Hoewel we in dit hoofdstuk alleen de mogelijkheid tonen om concentratieprofielen in een gel te manipuleren, zijn we begonnen met het aantonen van de potentie van deze techniek om 3D-celculturen te beïnvloeden, wat in het volgende hoofdstuk beschreven wordt. Naast 
biologische toepassingen kan onze techniek dienen als een algemeen microfluïdisch hulpmiddel voor het vertalen van de soorten oppervlaktepatronen die haalbaar zijn met laminaire stromingspatronen naar 3D-patronen in een hydrogelmatrix. Onze methode zou bijvoorbeeld controle over de lokale stijfheid van gels in 3D mogelijk kunnen maken door het concentratieprofiel van een crosslinker in de gel te manipuleren.

\section{Hoofdstuk 3}

In hoofdstuk 3 gebruiken we de in hoofdstuk 2 ontwikkelde techniek om het concentratieprofiel in het interstitium rond een microvasculair netwerk te manipuleren. Onze techniek kan concentratieprofielen nabootsen die vaak worden gebruikt in de literatuur over organen op chip, maar kan gelijktijdige perfusie van het microvasculaire netwerk mogelijk maken. In de toekomst zijn we van plan om met deze techniek fijnmazige microvasculaire netwerken te ontwikkelen en de effectiviteit ervan te tonen voor het modelleren van relatief complexe biologische fenomenen, zoals het effect van pluimen van groeifactoren op microvasculaire vaatsystemen.

\section{Hoofdstuk 4}

De techniek in hoofdstuk 4 maakt relatief goedkope en robuuste kwantificering van de diffusiviteit van eiwitten mogelijk. De uitgebreide interne kwaliteitscontroles die erin worden beschreven, maken een eenvoudige diagnose van problemen met de implementatie mogelijk en zorgen ervoor dat de uiteindelijke resultaten van hoge kwaliteit zijn. Om de effectiviteit van deze aanpak aan te tonen, hebben we een relatief nietgeoptimaliseerde opstelling geïmplementeerd en de diffusiviteit van een panel van veel voorkomende cytokines gemeten. Na het toepassen van kwaliteitscontroles, levert onze opstelling diffusiewaarden op die binnen het verwachte literatuurbereik vallen, wat de robuustheid en nauwkeurigheid van de techniek aantoont.

\section{Hoofdstuk 5}

In hoofdstuk 5 hebben we aangetoond dat de vaak omslachtige taak van het multiplexen van microfluïdische chips kan worden vereenvoudigd door een multiplexer in een chiphouder op te nemen in het ontwerp. Met ons chiphouderontwerp laten we zien controle te hebben over de druk in veel onafhankelijke uitgangskanalen met slechts twee ingangssignalen. We zijn vervolgens van plan aan te tonen dat deze chiphouder eenvoudig kan worden gekoppeld aan veel verschillende microfluïdische apparaten. 
Het gebruik van geïmpregneerde pakkingen om lekvrije en voorgespannen kleppen te maken, lost een groot deel van de beperkingen op van het circuitontwerp die gebruikelijk zijn bij het werken met elastomere kleppen. De mogelijkheid om grote drukken met kleinere drukken te schakelen, maakt de noodzaak van nauwkeurig afgestemde weerstandsnetwerken overbodig om een steeds afnemende druk aan de geschakelde zijde van de kleppen te leveren. Het aantal kleppen dat in een apparaat wordt gebruikt, kan daarom aanzienlijk worden opgeschaald met weinig ontwerpoverhead.

\section{Hoofdstuk 6}

In hoofdstuk 6 zagen we dat delaminatie van een 2D-materiaal van zijn substraat op zichzelf de algemeen waargenomen door spanning veroorzaakte doorslag van zwevende 2D-materiaalsystemen kan veroorzaken. We bevestigden deze observatie via verschillende metingen, met name: cyclische voltammetrie, in-liquid atomic force microscopie (AFM) en witlichtreflectiemicroscopie.

Voor zowel geëxfolieerd als chemisch opgedampte (CVD) grafeen gesuspendeerd op een SiN-membraan met een enkele nanoporie, hebben we de afbraak van het gesuspendeerde materiaalsysteem bij $\pm 200 \mathrm{mV}$ waargenomen. Via in-liquid AFM hebben we bevestigd dat een verhoogde blaar op micronschaal in het CVD-grafeen, die lokale delaminatie aangeeft, inderdaad boven de SiN-porie verschijnt wanneer de doorbraak wordt waargenomen. Vervolgens hebben we via coincident optische en amperometrische metingen vastgesteld dat, bij gebruik van CVD-grafeen, het gebied van de gedelamineerde patch sterk gecorreleerd is met de geleidbaarheid van het systeem tijdens de doorbraak. Vervolgens hebben we aangetoond dat delaminatie de enige oorzaak van afbraak kan zijn door herhaaldelijk doorbraak van een systeem op een monster van geëxpandeerd grafeen zonder waargenomen geleidende defecten te induceren en om te keren.

Deze resultaten tonen aan dat spanningsgemedieerde delaminatie een echt probleem kan zijn en waarmee rekening moet worden gehouden bij het ontwerpen van systemen die ondersteunde 2D-materialen gebruiken en spanningstoepassing vereisen. In het specifieke geval van zwevende 2Dmaterialen moeten stappen worden ondernomen om de hechting op het substraat te verbeteren wanneer hogere transmembraanspanningen moeten worden toegepast. 



\section{Appendices}

Supplementary information for chapters two four and six are located in the following appendices. 



\section{Appendix 1:}

Chapter 2 Supplementary Information 


\section{Calibration}

Before a chip is used, a calibration experiment is first run to determine the fluidic resistance values referred to in the Theory section.

For the calibration, the middle inlet reservoir is first loaded with the same fluorescent dye used throughout. Then, the two side reservoirs are loaded with buffer and $60 \mathrm{mBar}$ pressure is applied equally to all reservoirs. The pressure applied to the middle inlet reservoir is then reduced until the middle stream is seen to reduce to nothing. At this point the flow is stagnated in the middle channel so the pressure at the junction must be equal to the pressure applied to the middle channel. Under these conditions, if the flowrate through the main channel can be determined then its resistance $\left(R_{4}\right)$ can be calculated trivially via:

$$
\text { A1.1: } \quad R_{4}=\frac{P_{j}-P_{o}}{Q}
$$

Where $P_{j}$ is the pressure at the junction, $P_{o}$ is atmospheric pressure and $Q$ is the flow rate in the main channel.
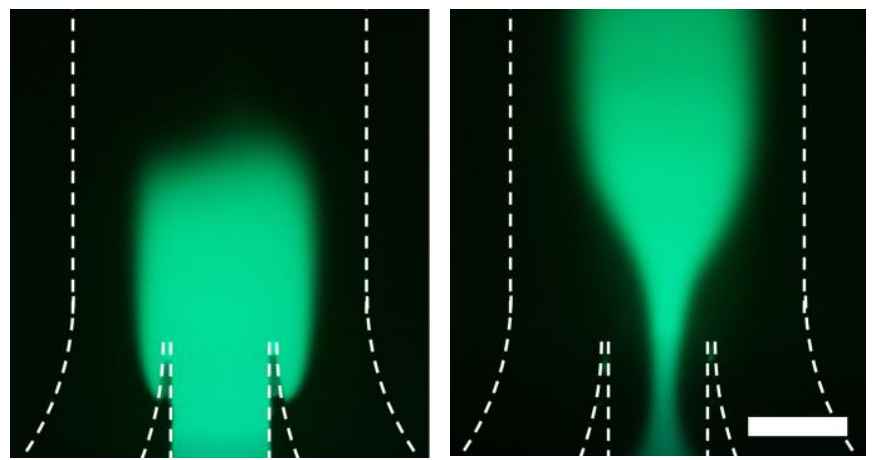

Figure A1.1: Sending a plug of dye through the chip for calibration of inlet pressures. (left) The plug of dye moving through the channel with inlet pressures of $60 \mathrm{mBar} 80 \mathrm{mBar}$ and $60 \mathrm{mBar}$ from left to right. The velocity of the plug during this part of the test is used to determine the resistance of the central inlet after all other resistances are determined. (right) The plug 40 seconds later after switching the central inlet back to the stagnation pressure. After the flow in the central inlet has fully stagnated, the velocity of the plug is used to determine the side inlet resistances and the resistance of the main channel. 
To measure the flowrate we introduce a short plug of dye into the channel before returning to stagnation conditions. This is achieved by sending a quick ( 30 second) pulse of high pressure ( $80 \mathrm{mBar}$ ) to the middle reservoir before returning it to the stagnation pressure. This results in a wide plug of dye entering the device as shown in Figure A1.1. Once the pressure returns to stagnation the velocity of the plug through the chip is measured to determine the flowrate and $R_{4}$ is calculated.

This test also tells us the combined effect of the side inlet resistances $R_{1}$ and $R_{3}$ and we can write

$$
\text { A1.2 } \quad R_{1}+R_{3}=\frac{P_{i n}-P_{j}}{Q}
$$

Where $P_{\text {in }}$ is the pressure at the inlets.

To make use of this, the ratio of $R_{1}$ to $R_{3}$ is determined by observing the percentage of the channel taken up by each inlet's stream during the stagnation part of the calibration. Consider the equation for flow rate through an arbitrary inlet channel:

$$
\text { A1.3 } Q_{n}=\frac{P_{i n}-P_{j}}{R_{n}}
$$

Where $Q_{n}$ is the flow through the nth inlet, and $R_{n}$ is the resistance of the nth inlet channel. The ratio of $Q_{1}$ to $Q_{3}$ can then be expressed as:

$$
\text { A1.4 } \quad \frac{Q_{1}}{Q_{2}}=\frac{R_{2}}{R_{1}}
$$

Which is directly measurable as the ratio of the stream widths and via equation A1.2 can be used to determine $R_{1}$ and $R_{3}$ as:

$$
\begin{array}{ll}
\text { A1.5 } & R_{1}=\left(1+\frac{Q_{3}}{Q_{1}}\right) \frac{P_{i n}-P_{j}}{Q} \\
\text { A1.6 } & R_{3}=\left(1+\frac{Q_{1}}{Q_{3}}\right) \frac{P_{i n}-P_{j}}{Q}
\end{array}
$$

Finally the resistance of the central inlet must be determined. We do this using the measured flowrate during the pulse and equation A1.3 for the central inlet. Notice that all the variables are determined except for the resistance of central inlet channel $R_{2}$ which can thus be solved for easily. 
After these simple calculations, all resistances in the system are known and equation 2.4 may be used to determine the flowrate through any or all inlet channels for a given set of inlet pressures.

Note that this calibration chiefly corrects for error during collagen filling of the of the inlet channels. The devices are filled from the center inlet and inevitably one inlet ends up slightly more filled than the other. This results in an average deviation of resistance from inlet to inlet of $8 \%$ (with a maximum of $12 \%$ ) in our case.

\section{Diffusivity}

The diffusivity of the labeled dextran was empirically determined in our gel using the 2D gel filled chips. For this experiment the flows were configured such that the right half of the channel was initially filled with the dye. The boundary was initially kept sharp by using a relatively high flow velocity $(60 \mu \mathrm{m} / \mathrm{s})$ then allowed to freely diffuse by abruptly stopping the flow. As the dye diffused we measure the profile at two time points and fit the single sided version of equation 2.8 (shown here as A1.6) for $D$.

$$
\text { A1.6 } \quad c_{t}(x, t)=c_{o} \operatorname{erf}\left(\frac{x}{\sqrt{4 D t}}\right)
$$

\section{Width Control}

Figure A1.2 compares the measured width of the concentration profile with the width predicted by equation 2.9 plotted against the target stream width instead of the target profile width. Notice that the predicted curve is consistently below the measured data for target widths greater than 150 microns. Note that while this is unexpected and interesting, it does not seem to detrimentally affect the controllability of the chip and could be calibrated for in future work.

We propose two possible explanations of the observed difference between theory and model, though both would require further work to validate.

First, the difference between data and theory may come from fabrication defects in the chips. Thin negative features were not successfully reproduced during lithography which resulted in unsuccessful bonding in the affected regions. While this left most of the chip geometry unaffected, the walls between the inlet channels have clearly not bonded (Figure A1.2 right, white arrow). Close inspection shows that at the junction, these walls do not 

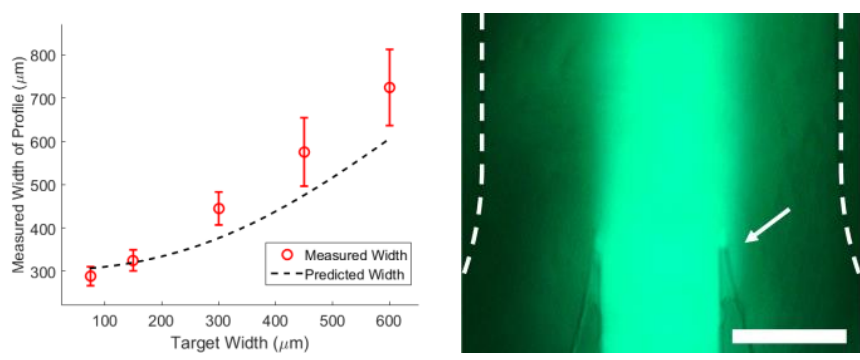

Figure A1.2: Unexpected increased width of concentration profiles. (left) Same data as displayed in Figure 2.3 with the width predicted by equation 2.9 plotted as well. Notice that at lower target widths, the theory matches the measurements but for higher target widths, the theory predicts a thinner profile than measured and error bars are wider. (right) Image of a device during a width test. Note that the dye spills over the walls between inlet channels slightly where marked with the arrow. Dotted lines represent the walls of the main channel, scale bar is $500 \mu \mathrm{m}$.

reach the full height of the channel thus allowing the flow from the central channel to bleed into the side channels before the junction. If this bleed happened uniformly over the whole channel depth there would be no problem, but because some of the designed wall is clearly still present in the device, the bleed cannot be uniform. More specifically, the central stream displaces the side streams more near the PDMS glass interface. This would lead to a non-plug profile if viewed in cross section and would result in an apparent widening of the concentration profile.

For the second potential explanation, consider the time evolution of the width at half max of a diffusing plug. As opposed to a diffusing Gaussian, the width at half max of a diffusing plug first shrinks as the flat high concentration region reduces in width. Thus one interpretation of the data shown in figure A1.2 is that the dye is significantly less diffusive in the direction perpendicular to the flow in the shown experiment when compared to our experiment used to determine the diffusivity of the dye. This may be possible. The experiment used to determine the diffusivity was performed under static conditions whereas the data shown in A1.2 was acquired under flow. Flow can cause the gel to rearrange on a microscopic level and pores can tighten in the direction perpendicular to the flow. As steric hindrance has a significant effect on the diffusivity of large dyes for the gel concentrations used, the diffusivity 
of the dye may decrease significantly perpendicular to the flow direction leading to the observed wider-than-modeled width at half max.

\section{Taylor Dispersion:}

We also considered the potential effects of Taylor dispersion in our system. While this would only affect diffusion in the direction of flow, we felt it important to consider when as it may negatively impact the resolution of the technique during temporal changes in the concentration profile as in the profiles shown in Figure 2.4.

Here we base our assumption on existing literature (Phillips 1990) [35] where Taylor dispersion in fibrous media is extensively discussed and modeled. They state, with reference to the older work of Durlofsky, [36] that hydrodynamic effects on the diffusivity can be neglected when the volume fraction of the media fall below $5 \%$. In our case we have a $4 \mathrm{mg} / \mathrm{mL}$ gel and, assuming that the reported specific volume of collagen of $1.89 \mathrm{ml} / \mathrm{g}$, (Levick 1987) [37] we come to a volume fraction of $0.76 \%$ well below the $5 \%$ limit.

\section{Long Term Stability:}

To investigate the long term stability of the system, we setup a fixed concentration profile and maintained it for several hours without adjusting the calibration. While these results are preliminary, the results were consistent with the lack of significant deviation during the experiments shown in figure

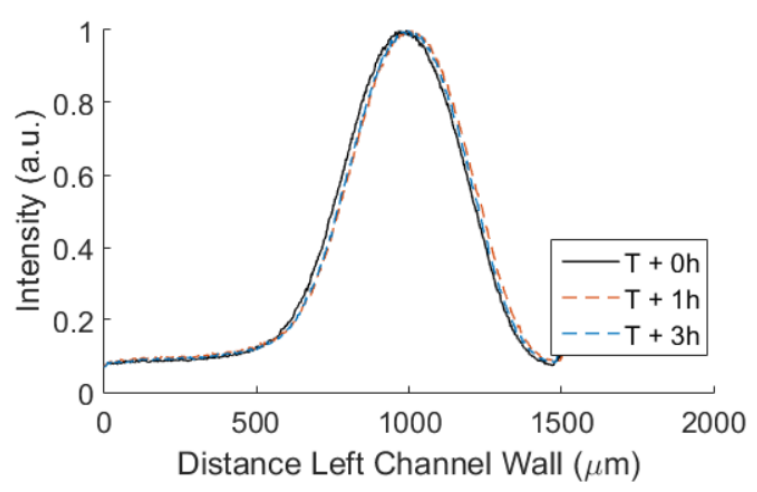

Figure A1.3: Fluorescence intensity profiles from long term stability experiment. Black curve is the initial measurement taken $10 \mathrm{~min}$ after flow was started. The red and blue dotted curves show the profiles 1 and 3 hours after the beginning of the experiment respectively. 
2.2 and 2.3 (these experiments were run in randomized order). The experiments shown in figure A1.3 indicate a drift of $2 \%$ in the peak position over the time tested. It is worth noting that that the majority of that drift took place within the first hour and over the next 2 hours the measured drift was $0.3 \%$

\section{Video SI-1:}

Video of the time response experiment. Green channel has been subtracted from red channel and contrast enhanced to highlight the faster diffusion of the red dye when compared to the green. At the end of the video, the flow is stopped and the profile allowed to fully diffuse. The video has been sped up slightly. The original capture rate is one frame per $4.25 \mathrm{sec}$.
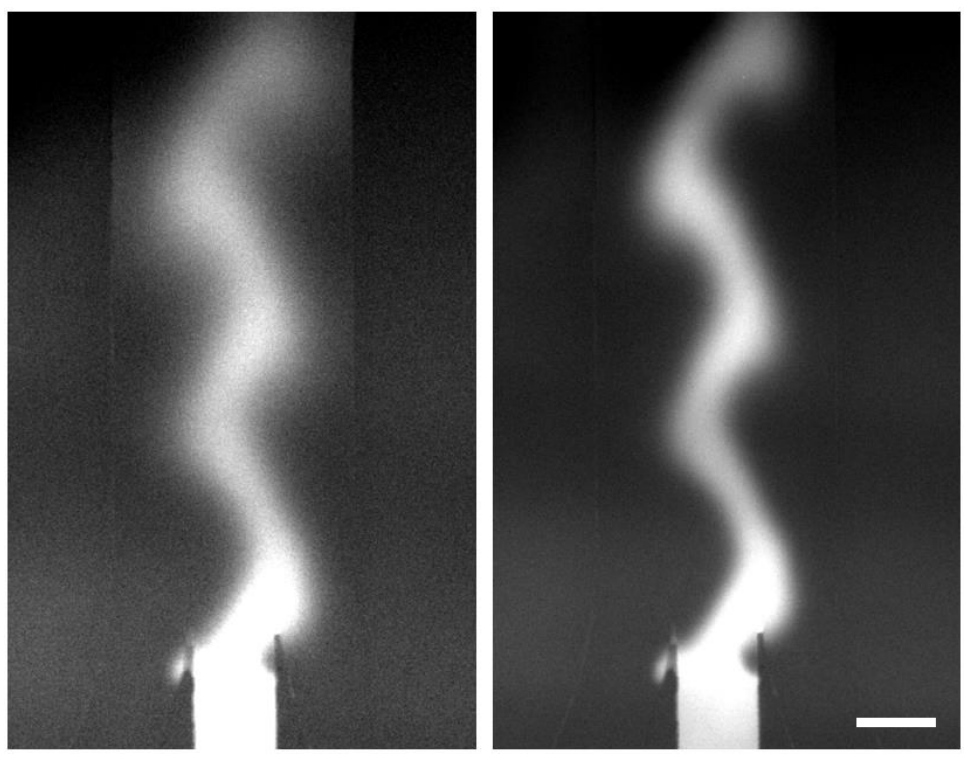

Figure A1.4: Unprocessed red and green channels from the image used to generate Figure 2.4 in the paper. The red channel is shown on the left while the green channel is shown on the right. Note that the diffusivity of the red dye is still obviously much more diffuse than the green before image processing. To generate the image in the paper, first a threshold was applied to remove the background. Then green image was subtracted from the red. Finally the images were recolored and combined. Scale bar is 500 microns. 




\section{Appendix 2:}

Chapter 4 Supplementary Information 


\section{Criteria Derivation}

To interpret the 2D profile as a translating 1D profile we must be sure that the 1D profiles are not affected by diffusion in the direction of flow. As diffusive flux goes with the gradient in concentration, this means that gradients in the flow direction must be much less than gradients perpendicular to flow. To estimate this, we calculate the rate of broadening of the profile from the diffusion length and compare it to the rate of downstream translation.

We start with the diffusion length for a 1D step function as an indicator of how broad the profile is at a given time:

\section{A2.1 $x=2 \sqrt{D t}$}

Its derivative then gives the rate of broadening at a given time:

A2.2 $\frac{\partial x}{\partial t}=\sqrt{\frac{D}{t}}$

The quantity to be minimized is the ratio of this broadening rate to the downstream translation rate (the velocity) and can be written as:

$$
\text { A2.3 } \frac{1}{V} \sqrt{\frac{D}{t}}<1
$$

The elapsed time of the 1D profile can be converted to the downstream position via the velocity and we finally arrive at equation 4.1 of the text:

A2.4 $\sqrt{\frac{D}{y V}}<1$

Use of equation 4.3 from the main text requires that the diffusing profile can be treated as if it were embedded in an infinitely wide system. Specifically, this is violated when the change in concentration at the bounding walls has changed significantly. From this we derive our criteria that the diffuse region of the profile is far from the walls of the channel. In this case, the diffusion length (A2.1) must be small compared to the width of the channel:

A2.5 $\frac{2 \sqrt{D t}}{w}<1$

After transforming time into downstream displacement we arrive at equation 2.2 from the text: 
A2.6 $\frac{2}{w} \sqrt{\frac{D y}{V}}<1$

\section{Transport Derivation}

To show that the diffusivity we recover is unaffected by binding and to justify our transposition of space and time, we provide here the full derivation of equation 4.3.

The analyte species concentration at a given point can be perturbed by three factors: diffusion, convection, and adsorption (binding) with the matrix. So we begin with the 3D convection-adsorption-diffusion equation for the free analyte and the equation for time evolution of the bound analyte:

A2.7 $\frac{\partial c_{f}}{\partial t}=\alpha D \nabla^{2} c_{f}-\alpha V \cdot \nabla c_{f}+R$

A2.8 $\frac{\partial c_{b}}{\partial t}=-R$

Where $c_{b}$ is the concentration of the bound analyte. In A2.7 both the diffusivity and the velocity are slowed by alpha, the fraction of time spent freely diffusing and convecting (as opposed to the fraction of time spent bound):

Expanding A2.7 we have:

A2.9 $\frac{\partial c_{f}}{\partial t}=\alpha D\left(\frac{\partial^{2} c_{f}}{\partial x^{2}}+\frac{\partial^{2} c_{f}}{\partial y^{2}}+\frac{\partial^{2} c_{f}}{\partial z^{2}}\right)-\alpha\left(V_{x} \frac{\partial c_{f}}{\partial x}+V_{y} \frac{\partial c_{f}}{\partial y}+V_{z} \frac{\partial c_{f}}{\partial z}\right)+R$

On the right hand side we make the following simplifications:

- $\quad \frac{\partial^{2} c}{\partial y^{2}}$ is set to zero as we have designed the experiments to ensure A2.3

- $\frac{\partial^{2} c}{\partial z^{2}}$ is set to zero as we have Darcy flow and thus no change in any profile in the $z$ direction

- $\quad V_{x}$ and $V_{z}$ are both also set to zero as flow is unidirectional

This leaves:

$\mathrm{A} 2.10 \frac{\partial c_{f}}{\partial t}=\alpha D \frac{\partial^{2} c_{f}}{\partial x^{2}}-\alpha V_{y} \frac{\partial c_{f}}{\partial y}+R$

If we assume that the system has had time to reach steady-state we can negate the left-hand side of both $A 2.10$ and A2.8. From equation A2.8 we now know that the adsorption term $(R)$ is also zero. This allows us to rewrite A2.10 as: 
$\mathrm{A} 2.110=\alpha D \frac{\partial^{2} c_{f}}{\partial x^{2}}-\alpha V_{y} \frac{\partial c_{f}}{\partial y}$

or:

$\mathrm{A} 2.12 \frac{\partial c_{f}}{\partial y}=\frac{D}{V_{y}} \frac{\partial^{2} c_{f}}{\partial x^{2}}$

Note that alpha has completely disappeared from the expression at this point. Any solution to this equation, will therefore depend only on the free diffusivity and the velocity and not at all on alpha. For a step function as the starting profile at $Y=0$, solutions to $A 2.10$ take the form: [REF CRANK]

A2.13 $\quad c_{-} f(x, y)=\frac{c_{o}}{2}\left(1+\operatorname{erf}\left(\frac{x}{2} \sqrt{\frac{V_{y}}{D y}}\right)\right)$

And, finally, as equation A2.11 contains no term for diffusive transport in the downstream direction, we realize that our solution is equivalent to a series of profiles defined in $x$, which simply translate downstream at the flow velocity. Therefore we can exchange $y$ for $t^{*} V_{y}$ and arrive at equation 4.3 from the text. Notably, equation 4.3 is simply the canonical solution to the diffusion of a 1D step function with a time offset added to account for extra diffusion near the junction.

In the next section, we discuss the possible effects of binding on the analysis.

\section{Binding Effects}

Here we demonstrate that binding only affects the measurement when the free concentration is close to saturating the matrix.

Assuming Langmuir kinetics, as long as binding is far from saturation and has had sufficient time to reach equilibrium, the bound concentration is expected to vary linearly with the free concentration and we can write:

A2.14 $c_{\text {total }}=c_{f}+c_{b}=c_{f}(1+k)$

Here $c_{b}$ is the bound concentration, $k$ is the ratio of bound analyte over free analyte and $c_{\text {total }}$ is the total observable concentration of analyte (free plus bound).

We can now simply substitute $(\mathrm{A} 2.13)$ for $\mathrm{c}_{\mathrm{f}}$ and we arrive at: 
A2.15 $c_{\text {total }}(x, y)=\left(\frac{c_{o}+k c_{o}}{2}\right)\left(1+\operatorname{erf}\left(\frac{x}{2} \sqrt{\frac{V_{y}}{D y}}\right)\right)$

Notice that this is the same as equation A2.13, except the constant $c_{o}$ has been replaced by $\mathrm{c}_{\mathrm{o}}+\mathrm{kc}_{\mathrm{o}}$.

During normalization, to correct for any bleaching, variation in light source intensity or change in optical path, we divide the intensity profile by a reference region in the high concentration stream. This is equivalent to dividing $A 2.15$ and $A 2.13$ by their respective maximum values. In both cases the result is the same:

A2.16 $c_{\text {norm }}(x, y)=\frac{1}{2}\left(1+\operatorname{erf}\left(\frac{x}{2} \sqrt{\frac{V_{y}}{D y}}\right)\right)$

All dependence on $\mathrm{k}$ has been removed, therefore no effect of binding can be distinguished.

However, in the case of binding concentrations near saturation, equation A2.14 no longer holds and saturation can cause significant and obvious deviation of the shape of $c_{\text {norm. }}$. While we do not use the following explanation to extract diffusivity from profiles with binding, we include it to help understand why the shouldering seen in figure 4.3 column 3 is expected.

In the case of binding near saturation, a non-linear relationship between bound concentration and free concentration is expected. We account for this using a Langmuir adsorption model:

A2.17 $\quad c_{\text {bound }}=\frac{c_{\text {max }} k_{e q} c}{1+k_{e q} c}$

where $c_{\text {bound }}$ is the concentration bound to the matrix, $c$ max is the concentration of available binding sites and $k_{\text {eq }}$ is the equilibrium constant for the binding. Now the expression for $\mathrm{c}_{\text {total }}$ is instead:

A2.18 $c_{\text {total }}(x, y)=\frac{c_{o}}{2}\left(1+\operatorname{erf}\left(\frac{x}{2} \sqrt{\frac{V_{y}}{D y}}\right)\right)+\frac{c_{\max } k_{e q} \frac{c_{o}}{2}\left(1+\operatorname{erf}\left(\frac{x}{2} \sqrt{\frac{V y}{D y}}\right)\right)}{1+k_{e q} \frac{c_{o}}{2}\left(1+\operatorname{erf}\left(\frac{x}{2} \sqrt{\frac{V_{y}}{D y}}\right)\right)}$

Which simplifies to: 
A2.19 $\quad c_{\text {total }}(x, y)=\frac{c_{o}}{2}\left(\frac{2 * c_{\text {max }} k_{e q}}{c_{o} k_{e q} \operatorname{erf}\left(\frac{x}{2} \sqrt{\frac{V_{y}}{D y}}\right)+c_{o} k_{e q}+2}+1\right)\left(1+\operatorname{erf}\left(\frac{x}{2} \sqrt{\frac{V_{y}}{D y}}\right)\right)$

Because A2.18 does not lend itself well to algebraic normalization, we provide a plot of a normalized profile with $c_{\max }$ and $k_{\text {eq }}$ taken to be near $c_{o}$ in figure 4.3 column c. where shouldering is readily apparent.

As this effect causes a fairly apparent disturbance of the profile when extreme, it can be used as a control on the basis of which the data can be rejected and a lower concertation can be used. Alternatively, when the effect is mild, it causes little to no perturbation of the slope at the center of the profile, thus leaving our recovered diffusivity unchanged.

\section{Velocity from Tracer Diffusion Profile}

It is necessary to know the flow velocity to recover the diffusivity of the analytes. Without it, there is no good way to use either equation A2.13 or equation 4.3 from the main text. In our present experiments we used FRAP to extract this parameter, but not every fluorescent microscope is so equipped. To remedy this, we provide another technique to extract the velocity from the diffusing profile of an added well characterized tracer dye.

One caveat to this method is that the velocity and the diffusivity of the tracer dye cannot be independently extracted by this analysis, so if the method below is used to extract the velocity, the sanity check described in figure 4.3 column 4 must be foregone. Therefore, this technique should only be applied in cases where: the velocity of flow is not known, devices are well trusted, and the free diffusivity of the tracer dye is well characterized.

To find the velocity from the profile, we will also use the slope at the center of the profile. We start with equation 4.5 but transform time back to space, again using the velocity as the conversion factor and end up with:

A2.20 $D=\frac{V_{y} c_{o}^{2}}{4 \pi \Delta y}\left(\frac{1}{c \prime_{2}^{2}}-\frac{1}{c \prime_{1}^{2}}\right)$

For a known $\mathrm{D}$ we can solve this equation for the velocity:

$\mathrm{A} 2.21 \quad V_{y}=\frac{4 \pi D \Delta y}{c_{o}^{2}}\left(\frac{c \prime_{1}^{2} c \prime_{2}^{2}}{c \prime_{1}^{2}-c \prime_{2}^{2}}\right)$ 
This velocity can be recovered much in the same as the diffusivity was in figure 4.2. A fixed frame can be picked for $y_{1}$ and $c^{\prime}{ }_{1}$ and the frame in which $c^{\prime}{ }_{2}$ is calculated can be swept over the profile. The velocity can then be recovered from this curve then be used for the analysis of the diffusivity of the analytes. 


\section{Appendix 3:}

Chapter 6 Supplementary Information 


\section{Setup}

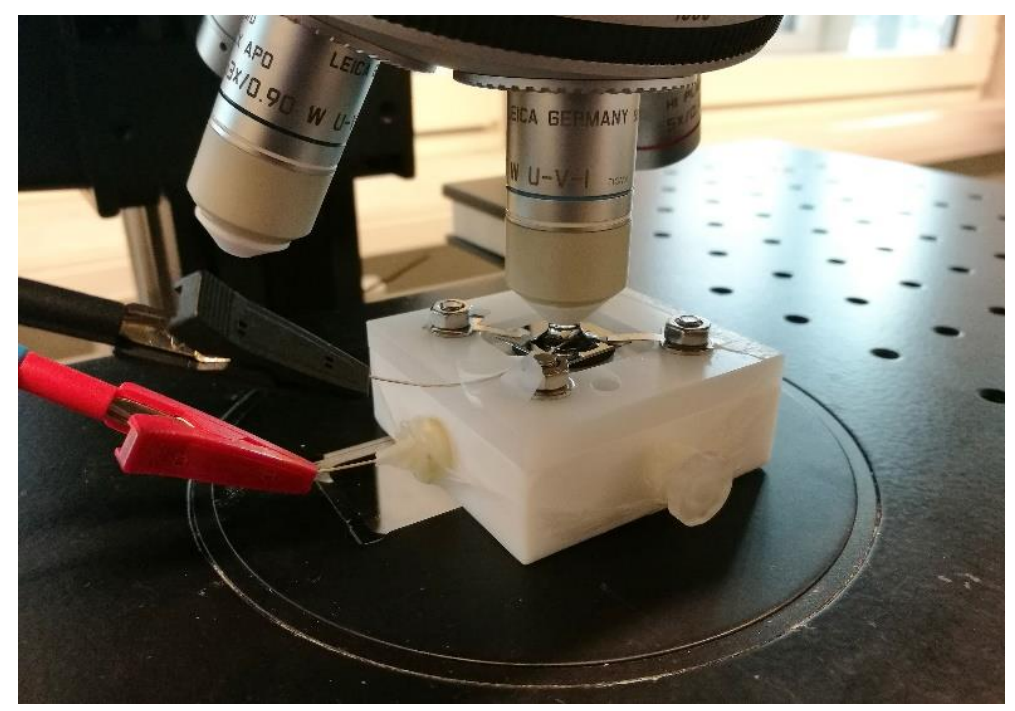

Figure A3.2: Photograph of the optical reflection microscopy setup, while performing current-voltage measurements. Two silver/silver chloride electrodes act as working and counter electrode.

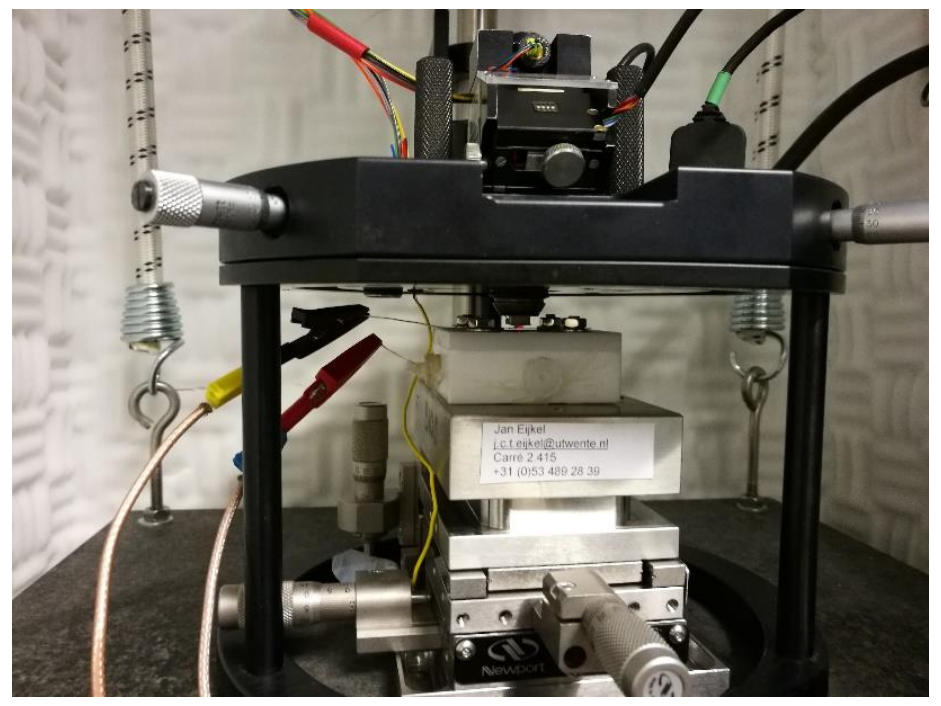

Figure A3.1: Photograph of the AFM setup, while performing current-voltage measurements. Two silver/silver chloride electrodes act as working and counter electrode. 


\section{Resistor Model}

In order to add some depth to our understanding of what happens in the system after delamination has occurred, we have derived a simple resistor model to describe the CVD graphene system as shown in figure A3.3. It should be noted that this model is a vast simplification and is only intended to indicate generally expected trends in the expected current and delaminated area. It does not account for a number of possible conflating factors including but not limited to: Ohmic voltage drop under the graphene as the current spreads, existing folds and buckling of the graphene, and local differences in the adhesion between graphene and the surface.

a
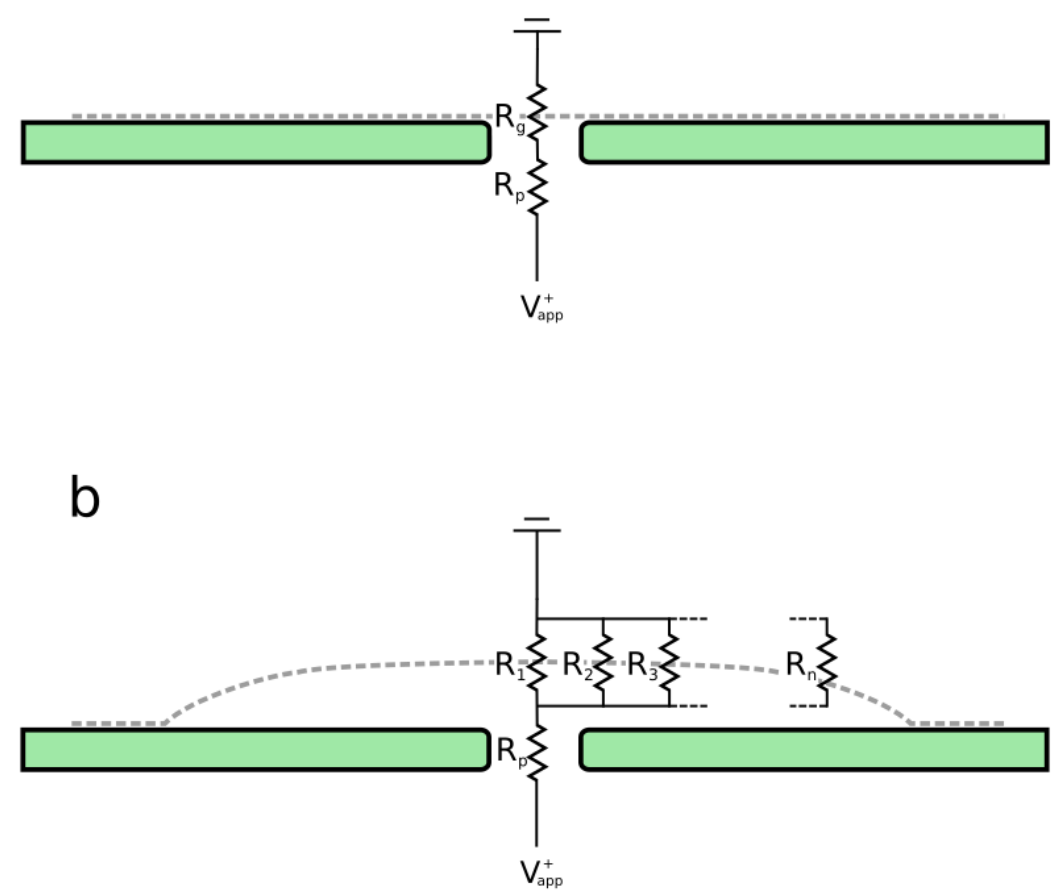

Figure A3.3: Showing the model used to predict general trends in system area and current. The green represents the SiN substrate, the dashed line represents the CVD graphene and the overlaid circuits are the models used for nondelaminated (a) and delaminated (b) predictions. 
In this model, we naively assume that the only relevant resistors are the SiN nanopore and the nanopore defects in the CVD graphene. If the voltage at the graphene is calculated to be below the threshold for delamination using the model for the non-delaminated system (Figure A3.3 a), then this model is considered valid and is used to calculate the current. Once the voltage at the graphene for the non-delaminated system (Figure A3.3 a) is calculated to be higher than the delamination threshold, the model for the delaminated system is used (Figure A3.3 b). In this case, the delaminated area and thus the number of exposed defects is taken to be that which would leave the voltage under the graphene at the voltage threshold for delamination. The exact implementation is explained in more detail below.

For a given applied voltage, the voltage at the graphene in the nondelaminated case is first calculated via Equation A3.1:

A3.1: $\quad V_{g}=\frac{R_{g}}{R_{g}+R_{p}} V_{a p p}^{+}$

Where $R_{g}$ is the resistance of the initially exposed graphene and $R_{p}$ is the resistance of the SiN nanopore as calculated by Equation $\mathrm{A} 3.2^{1}{ }^{2}$ :

A3.2: $\quad R_{p}=\frac{1}{\sigma}\left(\frac{4 w}{\pi d^{2}}+\frac{1}{d}\right)$

Where $\sigma$ is the solution conductivity taken to be $11 \Omega^{-1} \mathrm{~m}^{-1}$ for $1 \mathrm{M} \mathrm{KCl}^{3}$, w is the thickness of the $\operatorname{SiN}$ (40 nm in our case), and $d$ is the diameter of the nanopore (50 $\mathrm{nm}$ in our case).

If $V_{\mathrm{g}}$ is found to be below the voltage threshold for delamination (which we take to be $\sim 250 \mathrm{mV}$ in our system) then the current is calculated using the same model as:

A3.3: $\quad I=\frac{V_{a p p}^{+}}{R_{g}+R_{p}}$

\footnotetext{
${ }^{1}$ Vodyanoy, Igor, and Sergey M. Bezrukov. "Sizing of an ion pore by access resistance measurements." Biophysical journal 62.1 (1992): 10.

2 Hall, James E. "Access resistance of a small circular pore." The Journal of general physiology 66.4 (1975): 531-532.

${ }^{3}$ Lide, David R. "CRC Handbook of Chemistry and Physics, (CRC, Boca Raton, FL, 2003)." Google Scholar: 4-132.
} 
If, however the voltage at the graphene is found to be above the threshold for delamination then the model shown in (Figure A3.3 b) is used to calculate the current. In this case, the resistance of the graphene is calculated by considering the defects as parallel resistors:

A3.4: $\quad \frac{1}{R_{g}}=\frac{1}{R_{1}}+\frac{1}{R_{2}}+\cdots+\frac{1}{R_{n}}$

If each $R_{n}$ is taken to be associated with an area of graphene of the same size and the defects are expected to be randomly distributed, then all $R_{n}$ can be considered equal and $\mathrm{S} 4$ becomes:

A3.5: $\quad R_{g}=\frac{R_{1}}{n}$

If it is assumed that the delaminated area stops growing when $V_{g}$ equals the voltage threshold for delamination $\left(V_{t h}\right)$ then equation $A 3.1$ can be rewritten as:

A3.6: $\quad V_{t h}=\frac{\frac{R_{1}}{n}}{\frac{R_{1}}{n}+R_{p}} V_{a p p}^{+}$

And $\mathrm{n}$ can be found:

A3.7: $n=\frac{R_{1}}{R_{p}}\left(\frac{V_{a p p}^{+}-V_{t h}}{V_{t h}}\right)$

Here we consider $R_{1}$ to be the resistance associated with a single exposed $0.75 \mathrm{~nm}$ nanopore defect. (T. Jain, et.al. 2015). Finally, with all parameters determined, the current can be calculated again using A3.3.

Additionally, the delaminated area can be simply calculated. The spacing between pores is expected to be $70-100 \mathrm{~nm}$ (M. Boutilier et.al. 2014), we take the associated area to be to be $7200 \mathrm{~nm}^{2}$. The total delaminated area is simply calculated by multiplying this by the calculated $\mathrm{n}$.

Sample results are shown in figure A3.4, in which the same general trends can be seen in both experiment and model. 

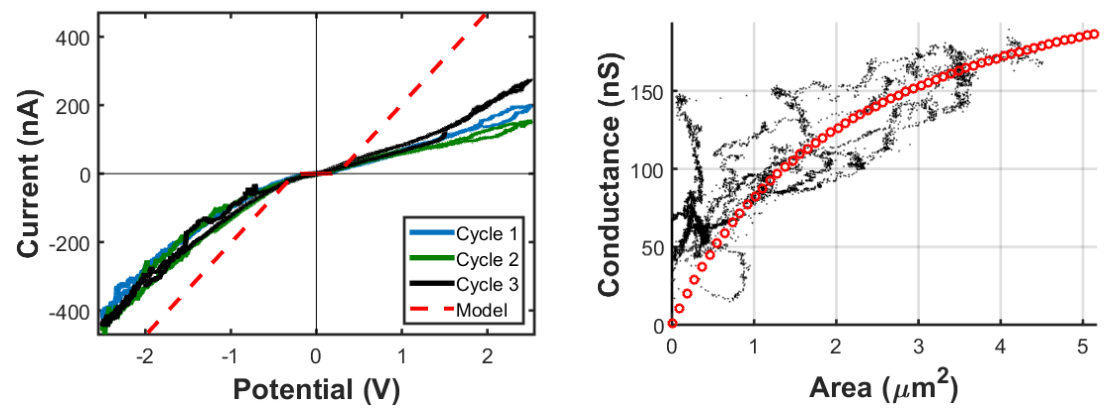

Figure A3.4: CV data from Figure 6.4(a-e) with modeling results using parameters mentioned in the text overlaid (left). Corresponding correlation between conductance and area with model prediction overlaid (right). Note that while the relation between voltage and current is not perfectly captured by the model, the general trend is strongly similar as is the case for the conductance-area relation.

\section{Chaotic Dynamics}

By looking at the limits of the model presented above, some of the chaotic dynamics of the system may also be understood. When solving, we effectively treat the resistance of the graphene per unit area as continuous ( $\mathrm{n}$ is allowed to assume decimal values) so that an incremental increase in area results in an incremental decrease of resistance. In the actual device this is not the case as the nanopore defects are discrete. This can lead to a situation where the sudden exposure of a new nanopore defect causes the voltage under the graphene to fall below the threshold for delamination. The resulting relamination can then hide the defect again, allowing the voltage to rise above the threshold once more. As the pore is cyclically exposed and hidden, discrete fluctuations in the current are expected. Such fluctuations in the current can indeed be seen in Figure 6.2 and may explain some of the chaotic behavior of these systems seen in the literature.[9,17]

\section{Current Under Pristine Graphene}

We note that the current seen when using pristine graphene is much less than that seen when using CVD graphene. We attribute this to a difference in the delaminated area between the CVD and pristine graphene. In the case of our pristine graphene device, the delaminated area will have to expand much further $(\sim 15 \mu \mathrm{m})$ to find the edge of the flake when compared to the two micron diameter observed when using CVD graphene (Figure A3.7). This 
implies that the current must travel further through a more confined conductive path and therefore we expect to see less current for the same applied voltage.

\section{SV1 and SV2}

The processed videos used to generate figure 6.4 (SV1 shows a-e while SV2 shows $f-j$ ) and the time evolution of the scatter plots. White circle indicates the area in which we expect the SiN nanopore.

\section{Raman Spectra}

Raman spectra of both CVD and Exfoliated graphene are shown below in figure A5.5 as proof of quality and to compare the defect peaks.

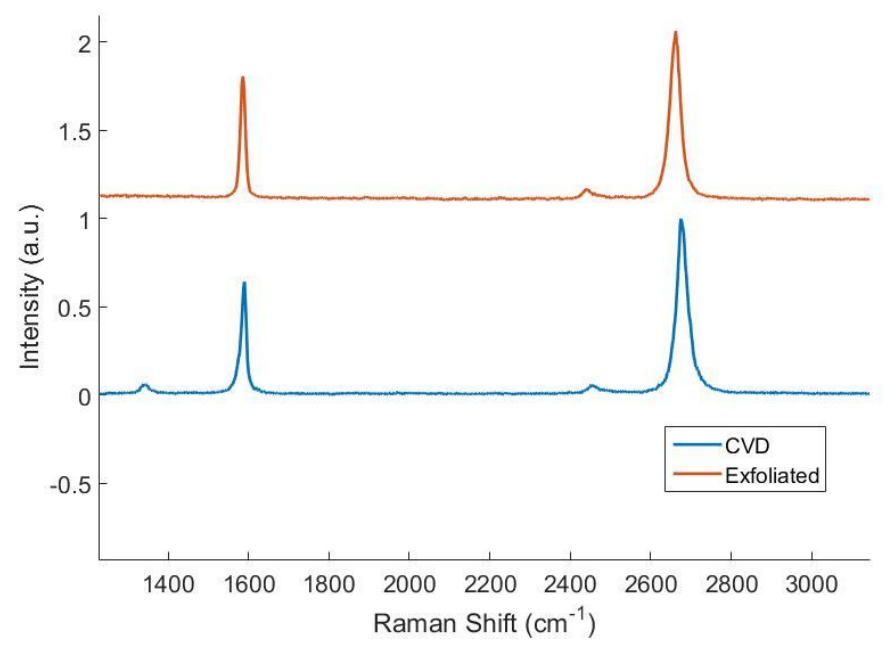

Figure A3.5: Raman spectra of CVD and exfoliated graphene on silicon oxide. The spectrum of exfoliated graphene has been artificially shifted upwards by 1.1 arbitrary units for ease of comparison. In particular, note the presence of a small but measurable defect peak in the CVD graphene spectrum near $1400 \mathrm{~cm}^{-1}$ and its absence in the exfoliated graphene's spectrum. 


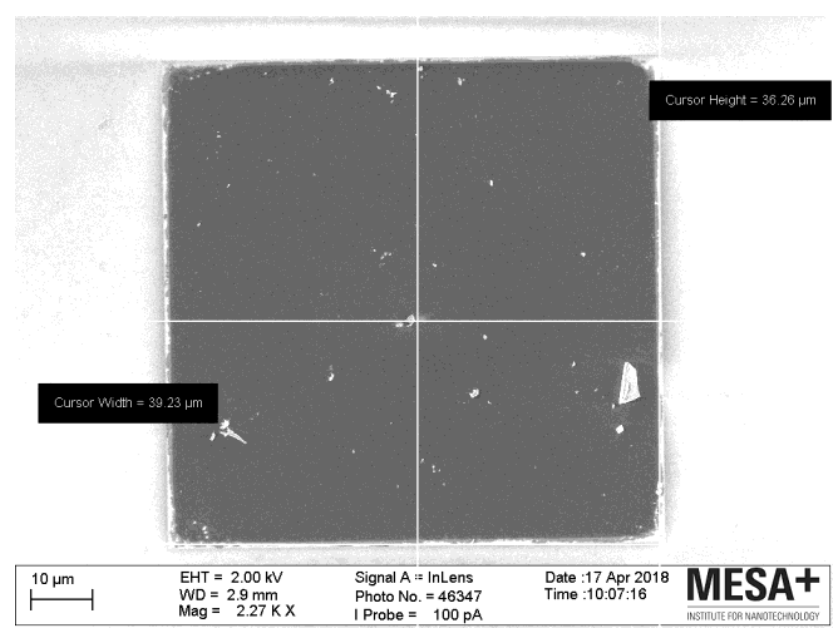

Figure A3.6: Sample SEM image of a SiN membrane used to locate the SiN nanopore. The SEM images were aligned with the optical and/or AFM images to locate the expected area of delamination. This technique allowed location of the nanopore with a confidence of $\sim 1$ micron as represented by the white circles in figs 3 and 4 .

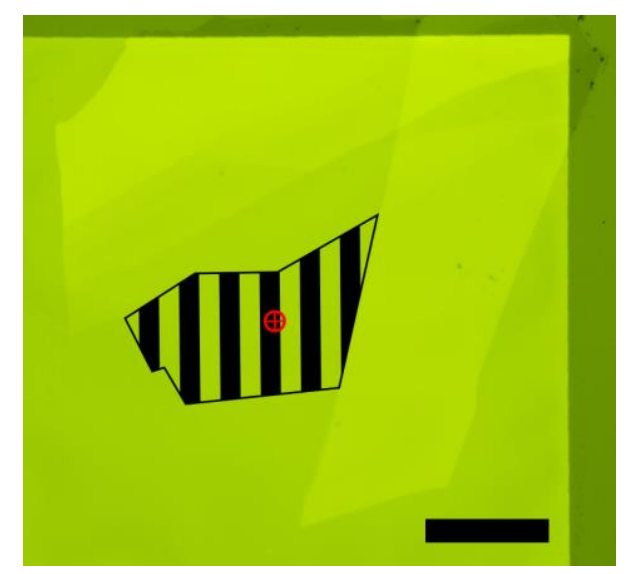

Figure A3.7: Optical image of pristine graphene placed on SiN membrane. The dark green area is that where the membrane is supported by the silicon substrate while the lighter green square is the suspended membrane. The lightest green patches are the exfoliated graphene with lighter green indicating more layers of graphene. The monolayer region is shaded with stripes and the location of the nanopore in the SiN membrane is indicated with a circle and crosshair. The flake of exfoliated graphene has been placed such that the nanopore is near the edge of the flake. Scale bar is 20 microns. 


\section{Publication List}

List of scientific output from this PhD 


\section{Journal Publications}

J. Loessberg-Zahl, A. D. Van der Meer, A. van den Berg, J. C. T. Eijkel, Flow Focusing Through Gels as a Tool to Generate Concentration Profiles in Hydrogel-Filled Microfluidic Chips, Lab on a Chip, 2019, 19, 207.

J. Loessberg-Zahl, D.S. de Bruijn, W.T.E. van den Beld, E. Dollekamp, E. Grady,A. Keerthi, J. Bomer, B. Radha, H.J.W. Zandvliet, A. A. Bol, A. van den Berg, and J.C.T. Eijkel, Voltage Mediated Delamination of Suspended 2D Materials Explains Commonly Observed Breakdown, Accepted by Physical Chemistry $\mathbf{C}$.

J. Loessberg-Zahl, M. Gillrie, R.D. Kamm, A. van den Berg, A. van der Meer, and J.C.T. Eijkel' Diffusion from Steady State Profile (DSSP) for Low Cost, Low Concentration Characterization of Protein Diffusivity, in preparation.

J. Loessberg-Zahl, Jelle Beumer, Albert van den Berg, Andries van der Meer and Jan C. T. Eijkel Flow Patterned Wettability for Rapid Prototyping of 3D Cell Culture Geometry, in preparation.

G. S. Offeddu, L. Possenti, J. Loessberg-Zahl, P. Zunino, J. Roberts, X. Han, D. Hickman, C. G. Knutson, R. D. Kamm, Application of Transmural Flow Across In Vitro Microvasculature Enables Direct Sampling of Interstitial Therapeutic Molecule Distribution, Small 2019, 1902393.

H. Le-The, M. Tibbe, J. Loessberg-Zahl, M.P. do Como, M. van der Helm, J. Bomer, A. van den Berg, A. Leferink, L. Segerink, J. Eijkel, Large-scale fabrication of free-standing and sub- $\mathrm{m}$ PDMS through-hole membranes, Nanoscale, 2018, 10, 7711.

C. Conner, T. de Visser, J. Loessberg-Zahl, S. Sherman, A. Smith, S. Ma, M. T. Napoli, S. Pennathur, D. Weld, Liquid Harvesting with a Liquid-Metal Microfluidic Influence Machinel,Physical Review Applied, 2018,9, 044008.

L. J. de Vreede, J. W. Berenschot, N. R. Tas, W. T. E. van den Beld, J. Loessberg-Zahl, A. van den Berg, J. C. T. Eijkel, Nanopores created using an internal shaddowmask process, IEEE 11th International Conference of Nano/Micro Engineered and Molecular Systems ,2016, 16505611. 


\section{Conference Contributions}

J. Loessberg-Zahl, M. Gillrie, R. D. Kamm, A. van den Berg, A. van der Meer, J. C. T. Eijkel, Diffusion from Steady-State Profile (DSSP) for Low Cost, Low Concertation Measurement of Diffusion, MicroTAS2019, 2019.

J. Loessberg-Zahl, D. Makkinga, A. van der Meer, A. van den Berg, J.C.T. Eijkel, Flow Throuhg Gels as a Tool to Generate 3D Concentration Profiles in Hydrogel-Filled Devices, MMB 2018, 2018.

J. Loessberg-Zahl, D. Makkinga, A. van der Meer, A. van den Berg, J.C.T. Eijkel, Flow Throuhg Gels as a Tool to Generate 3D Concentration Profiles in Hydrogel-Filled Devices, MicroTAS2018, 2018.

J. Loessberg-Zahl, W.T.E. van den Beld, A van den Berg, J.C.T. Eijkel, Electrowetting-Controlled Diode-Like Behavior of Suspended Graphene Membranes, Flow17, 2017.

J. Loessberg-Zahl, A. van der Meer, J.C.T. Eijkel, A. van den Berg, Customizable Patterning of Gel Structures for Cell Migration Studies and Organs-on-Chips, IOOSC17, 2017.

J. Loessberg-Zahl, D. Makkinga, A. van der Meer, J.C.T. Eijkel, A. van den Berg, Configurable Gel Geometry via Flow Patterning for Angiogenisis Assays, MicroTAS2017, 2017.

J. Loessberg-Zahl, J. C. Vollenbroek, A. van der Meer, J.C.T. Eijkel, A. van den Berg, Chipholder-Integrated 3D-Printed Pneumatic Logic Controllers for Microfluidic Chips, MicroTAS2017, 2017.

J. Loessberg-Zahl, A. van der Meer, J.C.T. Eijkel, A. van den Berg Angiogenesis-on-Chip Platform with Customizable Geometry and Reduced Sprout-Wall Interactions, Micro and Nanotechnology in Medicine Conference, 


\section{Thank You!}

While it is my name on the front of the book, the work here wouldn't be possible without help from many minds and support from many hearts. The instrumental folks who came to my mind during this writing are named below. But, before I call out anyone in specific, I would like to specifically thank anyone I miss. I can assure you that any forgetfulness on my part is not an indication that your contribution was negligible. In fact, I'm sure it was not, and I would like to thank you especially for bearing with me.

I first would like to thank my direct supervisors Jan and Andries. Not just for taking the plunge and hiring me in the first place, but for helping to make the entire PhD process a friendly and engaging one. I'll miss the laughing tone of our weekly meetings and the joking debates about who was "officially" late this time.

Jan, I particularly enjoyed our debates, whether we were attacking a difficult transport problem or railing against an ethical dilemma. I appreciate your unique ability to remain balanced and open in contentions discussions dominated by strong opinions. You somehow also do this without losing your oversight. That has facilitated some of my favorite and most frank discussions in the last four years. I hope some of that skill has rubbed off on me and I look forward to more coffee corner physics and philosophy during my next contract. Thanks as well for the support during some of the more ethically trying parts of this degree, that really made the difference.

Andries, I have to thank you for being a persistent force of positive attitude and good discussion at our meetings and at the vessel lunches. Not only was your knowledge about the fundamentals a great help, but I really appreciate your uncanny ability to get the right people talking to each other to solve highly interdisciplinary problems. Going forward, I hope to have learned a bit of your skill for sniffing out that delicate balance between "interesting to the fundamentals community" and "physically possible."

Albert, Thank you for making BIOS the diverse and exciting place it is to work today. The strong sense of good sportsmanship and the goal of success through intense collaboration clearly comes from the top. It has 
been a pleasure to work in the team you brought together and I feel lucky for the chance to spend another two years at BIOS now.

To the other members of my committee, Prof. Delamarche, Prof. van Zonneveld, Prof. Lemay, and Prof. Karperien thank you so much for taking the time to read and digest this work. A particular thanks to Prof. Delamarche, and Prof. van Zonneveld for making the journey to Enschede to attend. I am very much looking forward to our discussion during the defense.

To the graphene team, thanks for running that marathon and keeping the faith. To our external collaborators: Harold, Edwin, Ashok, Radha, Ageeth and Eldad, a huge thank you for taking the time to let us into your labs and sharing your resources. The supply of specialist material and knowledge from each of you was critical to that research.

In particular, thanks to Wesley and Douwe for the persistently joking atmosphere and good science on the graphene project here at BIOS. Wesley, the Graphene project was first yours, of course, and I am very glad to have had the opportunity to work on it with you. The goofy puns and the "just one more, I promise" Friday afternoon experiments made working with you when I first come to BIOS an absolute treat. That work atmosphere was one of the main reasons that I asked to stay. Douwe, it was a pleasure to work with you both as your supervisor and as a peer. Your independence in research (and your love of terrible puns) made you a great fit for the graphene project. I greatly appreciate our ongoing discussions on experimental practices and modeling. While I can hopefully say "the PhD was..." in a few weeks, I look forward to seeing both of you around BIOS in the coming years.

To my students from Saxion: Jelle and Daphne. Thank you for the incredibly concentrated and diligent work. It was good to get to know you and pleasure to work with you. You deserve the best in your future careers.

To the technicians and researchers at BIOS: Johan, Jan, Paul, Hans, Lisette and Eddie. I have to thank you for being a persistent wealth of practical microfluidic, fabrication, and biological knowledge. I can't Imagine what BIOS would look like without your open doors and the thread of your expertise running through the generations of PhD students. In particular, thank you Johan for the work on the Graphene project. The pore and membrane manufacturing was key to the experiments that finally lead to publication. 
To Hermine, organizing so many professors and students seems like a technical challenge all on its own, I can't imagine the headache we must give you. Thanks for your happy "get it done" attitude and for your bureaucratic magic.

To the other BIOS professors: Wouter, Mathieu, and Loes. Your collaborative attitudes, open doors and breadth of expertise is what makes doing the highly interdisciplinary projects we have at BIOS possible. I had too many warm and engaging technical discussions with you all to count. Thank you! And I look forward to working with you more and hopefully giving back some in my post doc.

To Loes, Paul, Jan and the group in Wageningen (Michiel, Hugo, and Ludvik) thanks for welcoming me into your protoplast project! Both the team's chemistry and the work itself are terribly exciting and that's a lucky match. I can't wait to get started in January.

A huge thanks to Hugo, Marinke, Martijn, Anne, and Paul for teaching me some of the tricky art of working with cell cultures. When I started, I had zero practical skill in cell biology but through your help and patience, I have at least the basics down now. Thanks, as well to the cell lab users in general, for making the painstaking work of cell culture and chip seeding easier with good humor and (more or less) good music.

Thanks also to those who kept the mood at the Vessel lunches warm and conversational, and particularly to Wesley for taking them over. The breadth of practical knowledge shared at those lunches was a huge help when it came to getting experiments working. I hope that the new generation of Vessel (or organ on chip) lunches keep spreading those little bits of insider knowledge.

To Vasilis, Anke, Dodo and Corentin, thanks for your concentrated technical discussions (and also for the very silly ones). While this may be true of most people at BIOS, It was always particularly fun to come to the coffee corner and debate the cause of some new strange observation or idea with you. I also really enjoyed our philosophical/ethical discussions. I always came away learning something, or had my perspective broadened, especially on the topics where we don't quite agree. 
To Vasilis in particular, thanks for always being willing to go into the weeds on difficult transport problems and strange results. It was always a lot of fun to pick through the strange corners of an experiment or a theory and see what could be dredged up

Eiko, it has been a lot of fun to get into some of the finer points of transport theory in our discussions. I'm curious to see how far down the rabbit hole you go. Best of luck in Belgium.

To my office mates, Jorien Hai and Christina, thanks for making the office a warm place to come into even in the middle of the dark Dutch winters. I'm really glad to have sat in such an easygoing office with a good international mix of people. We laughed a lot, if mostly at each other. Christina, thanks for the tips and tricks on electrochemistry and persistent supply of amazingly silly cat related content. Hai thanks for the fascinating conversations on the physics of fabrication and for your stubbornly positive attitude over the last four years.

Jorien, you know what you've done.... No, just kidding. I can't say enough here to do you justice. When things have been hard or when they've been amazing, you've been there, consistent and persistent. To just touch the surface: You kept working on our half-finished home when I spent half a year on the other side of the planet and you have amazing (if sleepy) patience for my bad habit of midnight brainstorming. Working on (re)building our house together and the general adventure of learning what it takes to make things work has really been the highlight of the last four years of my life. The warm cozy feeling of home we make together underwrites all the work here and I would trade everything in this book for it in a heartbeat. I've always got your back and I'm really looking forward to finding our next steps together.

Thanks as well to the friends I met when I first arrived: JB, Miguel, Hai, Renee, and Afshan. The BBQs and lunches we had together made my first proper visit to Europe a colorfully multicultural one and made the landing soft. I'm glad to have met you all and I wish you the best of luck going forward.

To Jasper Esther Jeroen and Marlou, thanks for all the good times and horrible puns. I especially enjoyed our extensive conversations about Plants and Tempeh, the debates about the effects of quantum bridging and I don't think III ever quite get the refrain to purple brain out of my head. But 
seriously, it's been fun and I hope we can keep our (almost) monthly DnD games going, at least while everyone is still in town. And a special thanks to Esther for working some of her photoshop wizardry last-min on this document.

Allison and Burcu, thanks for the company during those late evening coffee breaks. Sharing stories then, both the good ones and the bad, helped make me feel more at home in a strange group. Allison, it has been particularly heartwarming to keep in touch with you and follow your adventures. Your wherewithal is inspiring and our charming conversations often remind me of the better parts of North American culture. Best of luck, and keep in touch!

A huge thanks as well to all the here un-named BIOS members. The community spirit at BIOS as well as the wealth of expertise there makes it a unique place to work, both for good atmosphere and productivity. You all make it that way, and I hope we all keep it that way.

To the folks at AST who made collaboration across campus so easy and friendly, thanks you very much! Yusuf, in particular, I appreciate our recent collaboration on the paper and wish you good luck in your postdoc.

To Roger Kamm and the so called "Kammsters" (Particularly: Gio, Tina, Luca, Marks, Anya, Cynthia and Jean Carlos) thanks for opening up your resources and knowledge to me when I came to visit last year. The time people took to explain even some of the most basic parts of their work are what made that visit valuable and I'm glad to see some work form that time reaching fruition now.

To Kjeld, a special thanks for all the time and war stories shared back in California. You found me more or less floundering in the lab and shared with me your hard-won framework for experimental design. That framework is still doing good work for me today. For that, for the good company and for kicking me over here to the Netherlands (for just one summer, for sure) thanks a ton.

To both Sumitas in my life, thanks for having faith in me when I didn't have much else going for me. Both of you are incredibly enthusiastic individuals and, while we've had our differences, I've learned a lot from you both. 
Sumita Pennathur, a huge thanks for letting me into your lab as a fumbling bachelor student. I have you and your notes to thank for most of my bedrock knowledge on fluid dynamics and electrokinetics. No matter how many times an experiment went amiss, your hype kept me going back into the lab and, of course, I have to give you a huge thanks for kicking me other to the Netherlands in the end as well.

Sumita Gosh, it has been an absolute pleasure to watch you grow up from just a few steps ahead and to provide whatever good (or otherwise) advice I could muster at the time. I cant say how exciting it is to see you out in academia doing your own research now. Beyond that, I have always valued you as an intellectual sparing partner, from our walks in highschool debating math, politics, and philosophy of mind, to our more recent debates on social issues. Even the times when we disagree strongly, I feel like I come away with a deeper understanding of whatever we were discussing, and as better person for it. I'm incredibly glad we've kept in touch across years and continents, to me you'll always been awesome.

To my friends from college, Andrew, Lindsay, and James. Thanks for all the good times and thanks for the kindness and friendship during the rough times. Andrew, it was crazy good luck that we roomed together freshmen year. I had a blast being partners in crime for those 4 crazy years in Santa Barbara and I'm very glad we've all stayed in touch.

To my parents and family, thank you for your support beyond reason, even and especially during what I can only imagine were some strenuous situations. Whether I was a young kid throwing a magnet through the back door or an ungainly young adult going through the harsh growing pains of learning to live on my own, you have encouraged me to keep exploring and push myself. Beyond everything else, thanks for making home what it is. I love you guys and I look forward to having more time to come visit now that the sprint of the $\mathrm{PhD}$ is over.

To Jorien's family, also a warm thanks. You took me into your house again and again, and in spite of my persistent lack of Dutch language, you always made it feel normal and cozy to be there. Thanks as well for your continued help and patience with our homebuilding effort. But, of course, thanks most of all for raising such a wonderful daughter, I never doubt that I'm lucky to have her with me. 